\title{
Tension, Compression, and Bending of Superelastic Shape Memory Alloy Tubes
}

\author{
Benjamin Reedlunn ${ }^{\mathrm{a}, *}$, Christopher B. Churchill ${ }^{\mathrm{b}}$, Emily E. Nelson ${ }^{\mathrm{c}}$, John A. Shaw ${ }^{\mathrm{e}}$, Samantha H. Daly ${ }^{\mathrm{d}}$ \\ ${ }^{a}$ Sandia National Laboratories, P.O. Box 5800, Albuquerque, NM, 87185, USA \\ ${ }^{b}$ HRL Laboratories, 3011 Malibu Canyon Road, Malibu, CA 90265, USA \\ ${ }^{c}$ Department of Biomedical Engineering, University of Michigan, Ann Arbor, MI 48109, USA \\ ${ }^{d}$ Departments of Mechanical Engineering/Materials Science 6 Engineering, University of Michigan, Ann Arbor, MI 48109, \\ $U S A$ \\ ${ }^{e}$ Department of Aerospace Engineering, University of Michigan, Ann Arbor, MI 48109, USA
}

\begin{abstract}
While many uniaxial tension experiments of shape memory alloys (SMAs) have been published in the literature, relatively few experimental studies address their behavior in compression or bending, despite the prevalence of this latter deformation mode in applications. In this study, superelastic NiTi tubes from a single lot of material were characterized in tension, compression, and pure bending, which allowed us to make direct comparisons between the deformation modes for the first time. Custom built fixtures were used to overcome some long-standing experimental difficulties with performing well-controlled loading and accurate measurements during uniaxial compression (avoiding buckling) and large-rotation bending. In all experiments, the isothermal, global, mechanical responses were measured, and stereo digital image correlation (DIC) was used to measure the evolution of the strain fields on the tube's outer surface.

As is characteristic of textured $\mathrm{NiTi}$, our tubes exhibited significant tension-compression asymmetry in their uniaxial responses. Stress-induced transformations in tension exhibited flat force plateaus accompanied by strain localization and propagation. No such localization, however, was observed in compression, and the stress "plateaus" during compression always maintained a positive tangent modulus. While our uniaxial results are similar to the observations of previous researchers, the DIC strain measurements provided details of localized strain behavior with more clarity and allowed more quantitative measurements to be made. Consistent with the tension-compression asymmetry, our bending experiments showed a significant shift of the neutral axis towards the compression side. Furthermore, the tube exhibited strain localization on the tension side, but no localization on the compression side during bending. This is a new observation that has not been explored before. Detailed analysis of the strain distribution across the tube diameter revealed that the traditional assumption of elementary beam theory, that plane sections remain plane, does not hold. Yet when the strain was averaged over a few diameters of axial length, the tensile and compressive responses input into elementary beam theory predicted the global bending response with reasonable accuracy. While it is encouraging that a simple model could predict the moment-curvature response, we recommend that beam theory be used with caution. The averaged strain field can under/over predict local strains by as much as two-fold due to the localized deformation morphology.
\end{abstract}

Keywords:

Phase Transformation, Beams and Columns, Constitutive Behavior, Finite Deflections, Stability and Bifurcation

\section{Introduction}

Shape memory alloys (SMAs), such as NiTi-based alloys (Nitinol), exhibit two remarkable properties, the shape memory effect and superelasticity. The shape memory effect is the material's ability to recover large mechanically-induced strains upon heating above a transition temperature. Superelasticity (or pseudoelasticity) refers to the material's ability, above a transition temperature, to recover large strains isothermally

\footnotetext{
*Address all correspondence to this author. Email: breedlu@sandia.gov
} 
during a mechanical load/unload cycle, usually via a hysteresis loop. The mechanism for superelasticity is a stress induced transformation from austenite (A) to martensite (M) during loading and a reverse transformation from $\mathrm{M}$ to A during unloading. The magnitude of the tensile strain recovery in a Nitinol polycrystal is between 5 and $8 \%$ in the low-cycle limit and near $2.5 \%$ in the high cycle fatigue limit.

The bending flexibility of SMAs has led to numerous applications in the consumer, civil, transportation, and biomedical engineering sectors. In the consumer sector, superelastic SMAs are used for their bending compliance in eyeglass frames, clothing, headphone headbands, and rugged cell phone antennas (Humbeeck, 1999). SMAs are being explored for vibration damping of civil engineering structures, using the superelastic hysteresis in bending (Dolce and Cardone, 2005; Choi et al., 2009). In recent years, SMAs have been extensively used in biomedical devices, where the strong trend towards minimally invasive surgery in medicine is often enabled by superelastic SMAs (Duerig, T. W. and Pelton, A. and Stöckel, D., 1999). Self-expanding stents and stent grafts comprise the largest fraction of existing SMA biomedical applications. These devices are often laser cut from NiTi tubes into a series of crowns and struts, which are locally bent to fit the stent inside a catheter. The catheter delivers the stent to the desired location in the body, where it expands and scaffolds the circumference of a tubular lumen. Other SMA bending examples include catheter guidewires, inferior vena cava filters, tissue ablation devices, retrieval baskets, birth control devices, endoscopes, intra-aortic balloon pumps, and biopsy forceps. In dentistry, superelastic SMAs are used as pretensioned orthodontic wires and root canal files that must bend to accommodate tortuous crevices (Duerig, T. W. and Pelton, A. and Stöckel, D., 1999; Morgan, 2004).

Despite the myriad of applications that employ superelastic SMA in bending, and despite the number of SMA bending models (we found 13) (Atanacković and Achenbach, 1989; Thier et al., 1991; Pelton et al., 1994; Plietsch et al., 1994; Berg, 1995b; Auricchio and Sacco, 1997; Raniecki et al., 2001; Rejzner et al., 2002; Purohit and Bhattacharya, 2002; Liew et al., 2004; Rajagopal and Srinivasa, 2005; Buratti, 2005; De la Flor et al., 2010), few careful pure-bending experiments exist in the literature. Traditional 4-point bending fixtures operate under the assumptions of small beam displacements and rotations, which are easily violated when used with slender SMA specimens. Under large displacements, undesirable axial loads tend to develop due to the support constraints. Also, curvature measurements are usually inferred from grip displacements or rotations. Measuring deformation from the grips is problematic with superelastic SMAs, since they frequently transform to martensite prematurely inside grips where stress concentrations exist. This causes grip slippage that makes the deformation measurement inaccurate, and it tends to mask any stress peaks associated with the onset of transformation. In effect, the very bending compliance that makes SMAs attractive for applications also creates experimental difficulties.

A number of researchers have built custom devices to apply pure bending moments to SMA wires with negligible shear and axial loads, but each study has certain limitations. Berg (1995a) performed one of the first pure bending studies on SMA wires in the published literature. Berg's work is notable because he bent his specimens roughly twice as far as other researchers, and he used an optical microscope to measure the curvature $(\kappa)$ rather than relying on the grip rotation. In Berg's fixture the bending moment $(M)$ was applied by hanging weights, resulting in a moment-controlled experiment. Unfortunately, this control mode was unstable when $\mathrm{d} M / \mathrm{d} \kappa$ was close to zero, so gaps exist in Berg's data when this occurred. Furthermore, it is well known that the strain rate (especially in uniaxial tension) has a significant impact on the stressstrain response during the $\mathrm{A} \rightarrow \mathrm{M}$ and $\mathrm{M} \rightarrow \mathrm{A}$ transformation (e.g. Chang et al., 2006). Consequently, the bending response depends on the curvature rate, but as Berg acknowledged, his fixture had little control over this quantity. In other work, Bundara et al. (2000) also constructed a custom pure bending fixture, where the moment was applied to SMA wires by hanging weights. The moment curvature relation they measured was not as flat as Berg's, so they were able to capture more data points, but the strain rate still was not controlled. Rejzner et al. (2002) avoided this problem by building a displacement-controlled custom pure bending fixture that integrated directly into a load frame, but this design suffered from friction and imparted a small axial load on the specimen. The axial load was considered negligible by the authors and a friction correction was made, but the method was somewhat unclear to us. Furthermore both Bundara et al. (2000) and Rejzner et al. (2002) measured the curvature from the rotation of the grips, and bent their specimens roughly half as far as Berg, such that the outer fibers did not fully transform to martensite.

In this study, we sought to combine some of the best features of the previous studies and add new measurement capabilities. 
1. Instead of wires, NiTi tubes with a larger outer diameter were used to accommodate large bending deformations without requiring an extremely small radius of curvature. Although not used in the experiments shown here, this also allowed us to flow fluid within the tube to perform experiments at various temperatures while leaving the outer specimen surface unobscured for infrared or optical imaging (see Churchill, 2010).

2. A custom pure bending fixture was built and integrated into a tensile testing machine, facilitating rotation-controlled experiments under large displacements and rotations.

3. Unlike previous studies, the tube specimens from the same material lot were also characterized in uniaxial tension and compression to better quantify the tension/compression asymmetry, identify its implications for bending, and to assess whether uniaxial data could be used to predict the tube's pure bending response.

4. Stereo digital image correlation (DIC) was used during all experiments to measure the deformation field of the tube specimens, which provided new information not found in the previous studies. DIC is a non-contact optical method used to measure full-field surface displacements of an object by tracking the specular pattern on the surface of a specimen (Sutton et al., 2009).

A further complicating issue for experiments on NiTi specimens is the possibility of strain localization during phase transformation. In pure tension, textured NiTi is known to exhibit strain localization and propagation during certain unstable portions of the superelastic response, and this has now been widely studied, at least at the macroscopic level. In NiTi wires, localization creates propagating necks along the specimen length (Lüders-like deformation) that are responsible for the distinct stress plateaus observed during stress-induced $\mathrm{A} \leftrightarrow \mathrm{M}$ transformations at sufficiently slow loading rates (Shaw and Kyriakides, 1995; Iadicola and Shaw, 2002b; Chang et al., 2006). In thin strip (polycrystalline) NiTi specimens, localization manifests as angled fronts, sometimes with finer criss-crossing fingers, that propagate during portions of stress-induced transformations (Shaw and Kyriakides, 1997, 1998). In NiTi tube specimens, transformation front patterns are helical in certain cases, and macroscopically cylindrical in other cases, both of which were first extensively studied using optical photographs ( $\mathrm{Li}$ and Sun, 2002; Ng and Sun, 2006; Feng and Sun, 2006). The helical patterns were later quantitatively measured using two-dimensional DIC (Favier et al., 2007). Perry et al. (2007) noted the occurrence of localized transformation during bending of a NiTi tube, but did not investigate further. In addition, the following are a few notable efforts to experimentally address the lattice-level details associated with strain localization. Brinson et al. (2004) used optical microscopy with an interference filter to observe the evolution of martensite in isolated grains prior to, during, and after localization during tensile testing of thin NiTi strips. Mao et al. (2010) used electron backscatter diffraction on NiTi tubes to measure the distribution of grain orientations. (Schmahl et al., 2004; Hasan et al., 2008; Young et al., 2010) performed synchrotron X-ray diffraction to measure martensite phase fractions and elastic lattice strains on either side of a transformation front in NiTi cold-rolled bars and fine grained wires during tension. Mehta et al. (2007) also collected synchrotron X-ray diffraction data while bending stent ligaments (annealed), and they highlighted certain untransformed regions that were not captured by their finite element model.

The implications of strain localization during phase transformation on bending behavior are largely unknown. Accordingly, our aim is to quantify the evolution of macroscopic strain fields and to connect the different deformation modes of tension, compression, and bending. We have not attempted diffraction experiments, similar to those mentioned above, to directly link the crystal lattice-level to the macroscopic behavior, although that would be a worthwhile, if challenging, endeavor. As will be shown, the macroscopic behavior is already rich enough to warrant its own study. For this purpose, we found stereo DIC particularly useful to provide more quantitative and higher resolution measurements than shown previously, leading to a clearer understanding of the macroscopic strain fields across these related modes. To our knowledge compression and bending of NiTi have not been studied before using DIC. DIC provided accurate local strain measurement in the specimen gage length without the need to resort solely to grip displacements or rotations. DIC clearly showed when strain localization did, or did not, occur. Furthermore, DIC allowed us to directly measure the evolution of the neutral strain axis for the first time during NiTi bending.

The heterogenous nature of the bending strain fields also raises the question whether pure tension and pure compression responses can be used in classical beam theory to accurately predict a NiTi beam's 


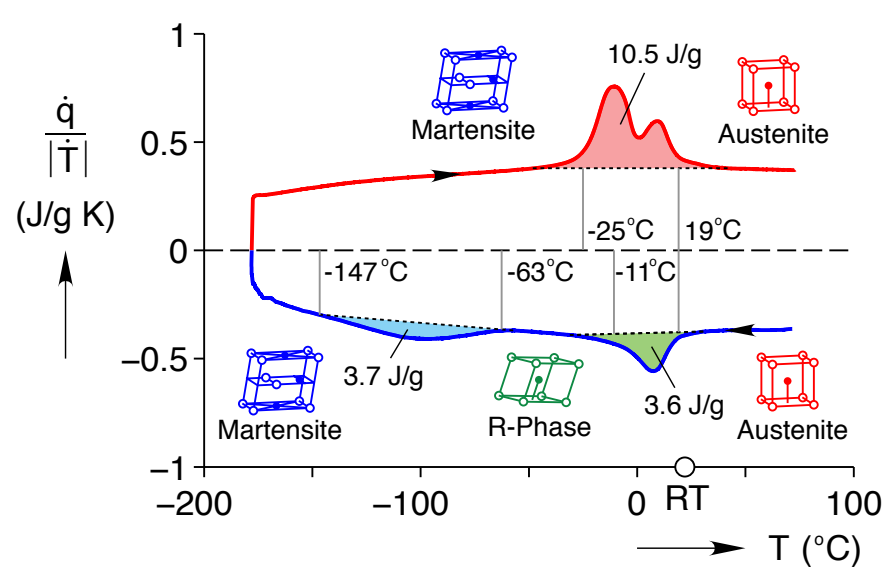

(a)

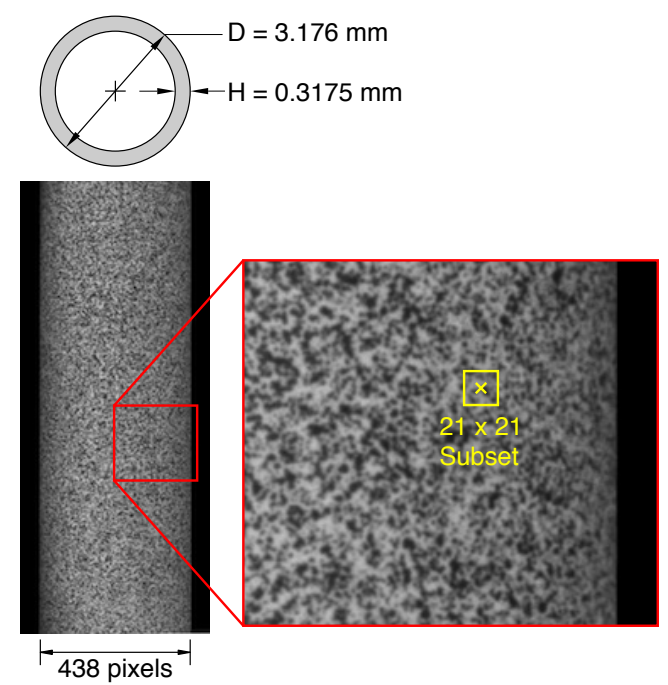

(b)

Figure 1: (a) Differential scanning calorimetry thermogram of NiTi tube material. (b) Cross-section dimensions and photograph of tube specimen showing the speckle pattern for tracking full field deformations.

moment-curvature response. Classical Euler-Bernoulli bending kinematics assumes a linear strain profile across the cross section, but as will be shown in Section 5, this does not hold true for bending of superelastic NiTi. Thus, elementary beam theory would seem to be invalid for this material, yet we show it can still be used in certain circumstances. The addition of uniaxial tension and compression data on material specimens from the same lot allowed us to directly compare these data sets with our bending data and to address when (and when not) to use elementary beam theory.

\section{Materials and Specimen Preparation}

The material studied was cold-drawn, slightly Ni-rich NiTi tube from Memry Corporation (P/N 10130W, Lot \# 957152). The outer diameter and inner diameter were measured as $D=2 C=3.176 \mathrm{~mm}(0.125$ inch) and $2.540 \mathrm{~mm}$ (0.100 inch), respectively, which corresponds to an outer diameter to thickness ratio of $D / H=10$. Differential scanning calorimetry (DSC), using a Q2000 DSC (TA Instruments) at a scan rate of $\dot{T}= \pm 10{ }^{\circ} \mathrm{C} / \mathrm{min}$, was performed to characterize the transformation temperatures. The DSC specimen was prepared by diamond saw cutting six $0.75 \mathrm{~mm}$ thick half circles from the tubes (specimen mass of $35.4 \mathrm{mg})$ and placing them flat in the sample pan to ensure good thermal contact. The austenite $\left(A_{\mathrm{S}}\right.$ and $\left.A_{\mathrm{f}}\right)$, martensite $\left(M_{\mathrm{S}}\right.$ and $\left.M_{\mathrm{f}}\right)$ and rhombohedral $\left(R_{\mathrm{s}}\right.$ and $\left.R_{\mathrm{f}}\right)$ transformation temperatures, as well as the corresponding specific enthalpies of transformation, are indicated on the thermogram in Fig. 1(a). Since $A_{\mathrm{f}}=19{ }^{\circ} \mathrm{C}$, the alloy is initially austenite at room temperature and is superelastic. During cooling, the material exhibits a two step transformation first $\mathrm{A} \rightarrow \mathrm{R}$ and then $\mathrm{R} \rightarrow \mathrm{M}$, as is common for commercial superelastic Nitinol alloys. During heating, the $M \rightarrow R$ and $R \rightarrow A$ transformations overlap creating a double peak.

New, as-received, tube specimens were used for each tension, compression, and bending experiment. The strain distribution during the experiments was measured by DIC, which requires a high contrast specular pattern on the surface of the specimen. The specimen was first airbrushed with a background coat of Golden Airbrush Titanium White (\#8380), followed by a speckle coat of Golden Airbrush Carbon Black (\#8040). The resulting speckle pattern is shown in Fig. 1(b). The magnified image is a portion of the actual image used for the DIC analysis and shows a typical $21 \times 21$ subset used in the correlation analysis. More details of the specimen airbrush procedure and subset selection can be found in Reedlunn et al. (2012). Segments of the specimen that were to be placed inside the tension and compression grips were not painted. 
Table 1: Parameters for tension (T), compression (C), and bending (B) experiments

\begin{tabular}{lllllll}
\hline & \multicolumn{5}{c}{ Experiment } \\
\cline { 2 - 7 } Parameter & $\mathrm{T} 1$ & $\mathrm{~T} 2$ & $\mathrm{C} 1$ & $\mathrm{C} 2$ & $\mathrm{~B} 1$ & $\mathrm{~B} 2$ \\
\hline$L(\mathrm{~mm})$ & 14.13 & 14.76 & 41.59 & 40.83 & $\geq 14.77$ & $\geq 14.77$ \\
\hline$L_{\mathrm{f}}(\mathrm{mm})$ & 14.13 & 14.76 & 9.38 & 7.87 & 14.77 & 14.77 \\
$L_{\mathrm{f}} / D$ & 4.45 & 4.65 & 2.95 & 2.48 & 4.65 & 4.65 \\
\hline$L_{\mathrm{e}}(\mathrm{mm})$ & 9.57 & 10.13 & 5.33 & 3.29 & 9.58 & 10.42 \\
$L_{\mathrm{e}} / D$ & 3.01 & 3.19 & 1.68 & 1.04 & 3.02 & 3.28 \\
\hline$\dot{\delta} / L\left(\mathrm{~s}^{-1}\right)$ & $\pm 2.18 \times 10^{-5}$ & $\pm 2.18 \times 10^{-5}$ & $\mp 2.18 \times 10^{-5}$ & $\mp 2.18 \times 10^{-5}$ & - & - \\
$\dot{\bar{E}}_{Z Z}^{\mathrm{B}}( \pm C)\left(\mathrm{s}^{-1}\right)$ & - & - & - & - & $\approx \pm 2 \times 10^{-5}$ & $\approx \pm 4 \times 10^{-5}$ \\
\hline
\end{tabular}

\section{Experimental Setups}

Sections 3.1 and 3.2 describe the setups for uniaxial tension, uniaxial compression, and pure-bending experiments. All experiments were performed in room temperature air. Six experiments, two of each type, will be presented in the following sections, and Table 1 provides a summary of specimen gage lengths and loading rates. Experiments are identified by T1, T2, C1, C2, B1, and B2, denoting the type of experiment by the letter and the number denoting the first or second experiment of that type. The reference length of the tube specimen is $L$ and its elongation is $\delta$. The viewable free length is $L_{\mathrm{f}}$, and the DIC "extensometer" (gage) length chosen is $L_{\mathrm{e}}$. The elongation rate (average strain rate) is $\dot{\delta} / L$ for the uniaxial experiments, and the estimated average outer-fiber strain rate during bending experiments is $\dot{\bar{E}}_{Z Z}^{\mathrm{B}}( \pm C)^{1}$.

\subsection{Tension and Compression Setups}

The tension and compression setups shown respectively in Fig. 2(a) and 2(b) were similar, except for the grip designs. Both setups utilized a Test Resources 830L load frame with a linear servomotor actuator, operated in displacement control. A $5 \mathrm{kN}$ load cell monitored the resultant axial force $(P)$. A $2825 \mathrm{~N} \cdot \mathrm{mm}$ torque cell was also included in the tensile setup, but the torque was too small to obtain a reliable reading (as was also found by Feng and Sun (2006)). The lower grip was fixed while the upper grip displacement $(\delta)$ was prescribed at a constant rate $(\dot{\delta}=d \delta / d t)$ during loading and unloading. To maintain isothermal conditions, the prescribed global strain rate was quite slow, $\dot{\delta} / L= \pm 2.18 \times 10^{-5} \mathrm{~s}^{-1}$, where $L$ is the length of the specimen between grips. Isothermal conditions were verified with two type-K thermocouples, one measuring the air temperature $\left(T_{\mathrm{a}}\right)$ and one measuring the specimen temperature $\left(T_{\mathrm{s}}\right)$. A small amount of thermally conductive paste (Omegatherm 201) was applied to the specimen thermocouple and the tube outer surface to ensure good thermal contact. Measured deviations in specimen temperature due to latent heat effects during transformation were less than $\pm 0.3^{\circ} \mathrm{C}$ from room temperature in both sets of experiments.

The tension specimens were held in $3.175 \mathrm{~mm}(1 / 8 \mathrm{inch})$ ER 16 steel sealed collets as shown in the section schematic of Fig. 2(a). The collet holder was bolted directly to the load frame, and a Teckniks Power Coat nut was used to compress the collet. The unpainted (gripped) segments of the tension specimens were scored with a file to provide a higher friction gripping surface. In addition, steel dowel pins (2.515 mm diameter) were inserted into the ends of the tube specimen within the gripped region to increase the radial stiffness. Care was taken to ensure that the dowel pins did not protrude into the gage section between the grips. Dowel pins were not used in the compression specimens, and the unpainted sections were coated with light oil to help the specimen slide freely in the compression grips. The compression grips shown in the section schematic of Fig. 2(b) were two $25.4 \mathrm{~mm}$ diameter steel cylinders, each with a $3.20 \mathrm{~mm}$ diameter by $15.9 \mathrm{~mm}$ deep hole. This design left a free length between the grips $\left(L_{\mathrm{f}}\right)$ for DIC measurements and permitted a relatively long specimen length $L$, while laterally supporting the specimen to avoid column buckling. Buckling was further prevented by the relatively stiff, short stackup (hardware between the crosshead and base in Fig. 2(b)) and careful alignment of the compression grips.

\footnotetext{
${ }^{1} \mathrm{~A}$ bar over a quantity refers to an averaged quantity. As described later, $E_{Z Z}^{\mathrm{B}}$ denotes the Biot strain along the $Z$-axis (centerline) of the tube.
} 


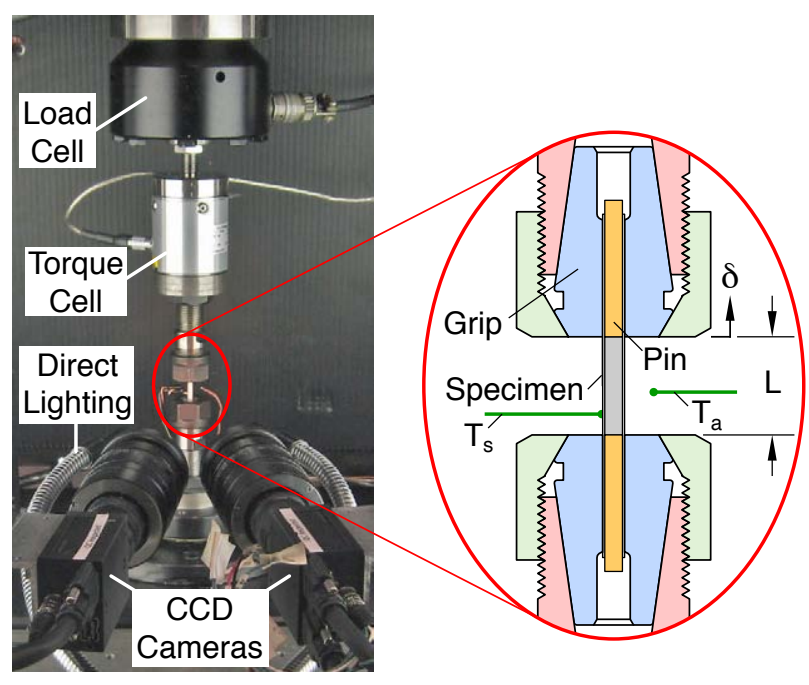

(a)

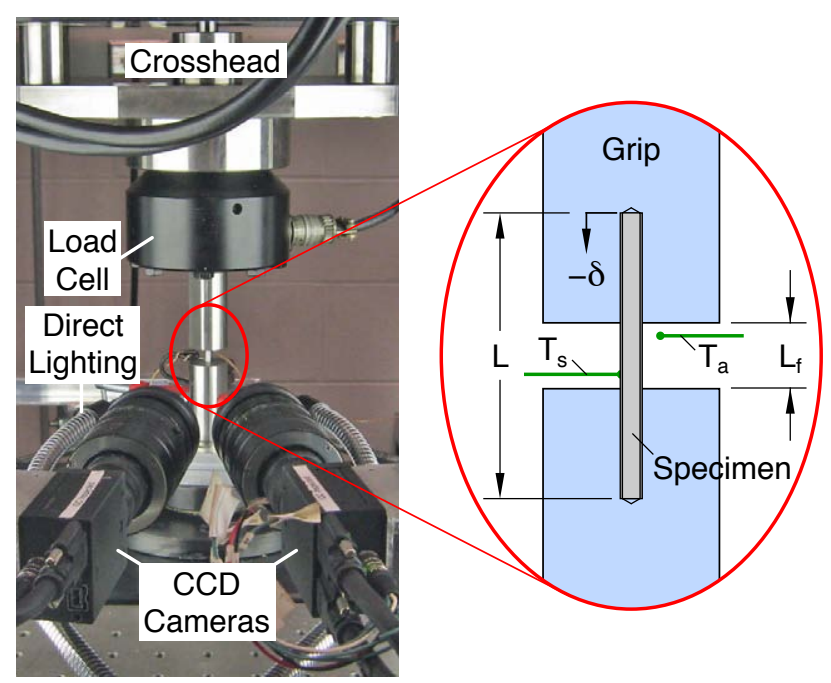

(b)

Figure 2: Photographs of uniaxial setups and schematics of grips (to scale): (a) tension setup, (b) compression setup.

\subsection{Bending Setup}

A photograph of the bending setup is shown in Fig. 3(a), along with a magnified view of the 4-point bend fixture. The bending fixture design is similar to that used in Kyriakides et al. (2008) for pipe bending, except that our bending fixture was scaled down and integrated into an Instron 5585 lead-screw driven load frame. One can see the basic operation of the fixture by comparing the close-up photo of the fixture in Fig. 3(a) with an undeformed specimen, against the schematic in Fig. 3(b) with a deformed specimen. The four loading points are $4.80 \mathrm{~mm}$ diameter stainless steel rollers, machined with a semi-circular relief to capture the tube in the bending plane. Each roller was press-fit into a pair of miniature ball bearings such that the rollers could rotate freely, allowing the tube to slide to avoid any axial tension. Similar to the compression specimens, a thin coating of oil was carefully applied to the unpainted ends of the tube to ensure that the tube would slide freely. Each pair of bearings was affixed to a $D_{\mathrm{w}}=23.86 \mathrm{~mm}$ diameter aluminum loading wheel. Each loading wheel was mounted to another center bearing. The ends of a steel cable were attached and wrapped around each of the two wheels. The cable was pulled from above by the load frame, operated under displacement control. A pair of overhead pulleys balanced the force between the wheels. A $500 \mathrm{~N}$ load cell, attached to the load frame crosshead, measured the vertical load $P$ as the load frame crosshead was raised by an amount $\delta$. The moment $M$ applied to the tube was $P\left(D_{\mathrm{w}}+D_{\mathrm{c}}\right) / 4$, where $D_{\mathrm{c}}=1.19 \mathrm{~mm}$ was the diameter of the steel cable. The initial length of tube between the rollers was $L=14.77 \mathrm{~mm}$, but since the axial load was essentially zero, the length of tube being bent became somewhat larger when endrotations were large. The average curvature was measured over a fixed length $L_{\mathrm{e}}$ of material data points as described below in Section 3.3. Ambient temperature $T_{\mathrm{a}}$ was measured by a type-K thermocouple, but due to the large displacements, the temperature of the specimen was not directly measured. To ensure nearly isothermal conditions, the rotation rate was quite slow. In the single load-unload bending experiment (B1), the strain rate at the outer tensile fiber of the tube was about $\pm 2 \times 10^{-5} \mathrm{~s}^{-1}$ (11.5\% strain over $\left.87.9 \mathrm{~min}\right)$, similar to the average strain rate of the uniaxial experiments. A slightly faster strain rate of $\pm 4 \times 10^{-5} \mathrm{~s}^{-1}$ at the outer tensile fiber was used for the second bending experiment (B2).

\subsection{Stereo DIC Setup and Post-processing}

Stereo DIC was performed to measure the local surface displacement and strain fields on the viewable portion of the specimen during all the experiments presented in Sections 4 and 5. In the tension and compression experiments, the mechanical responses are reported in terms of averaged strains $\left(\bar{E}_{Z Z}^{\mathrm{B}}\right)$ in a selected gage region $\left(L_{\mathrm{e}}\right)$ from the DIC analysis, rather than the global strain $(\delta / L)$, to eliminate any gripping artifacts. A sequence of simultaneous images was taken by the gray-scale CCD cameras shown in 


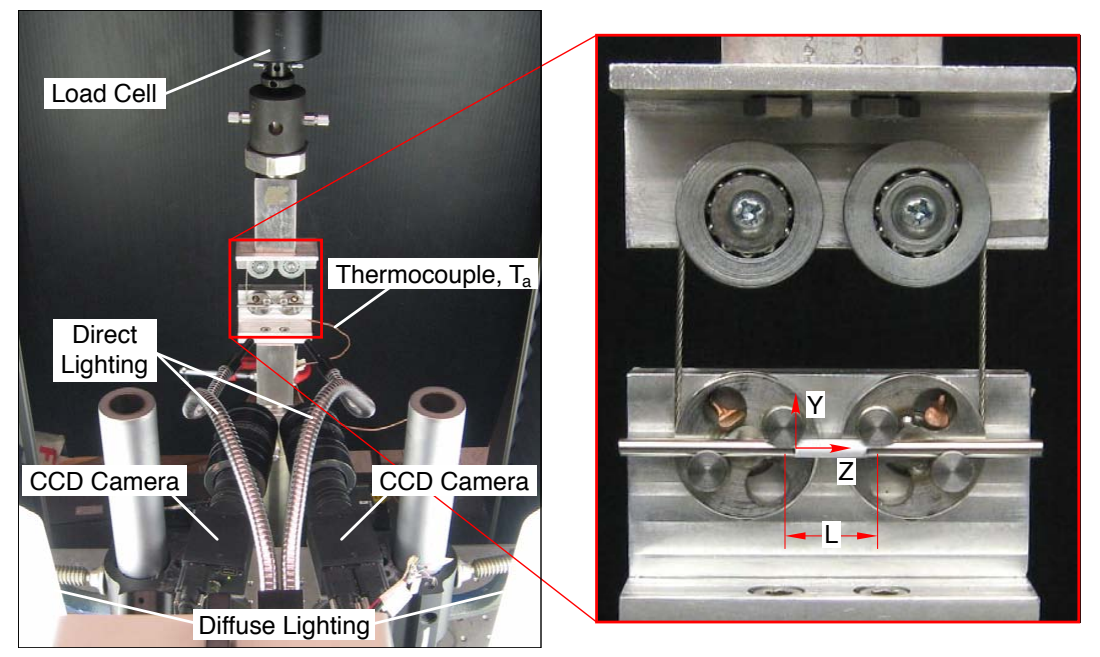

(a)

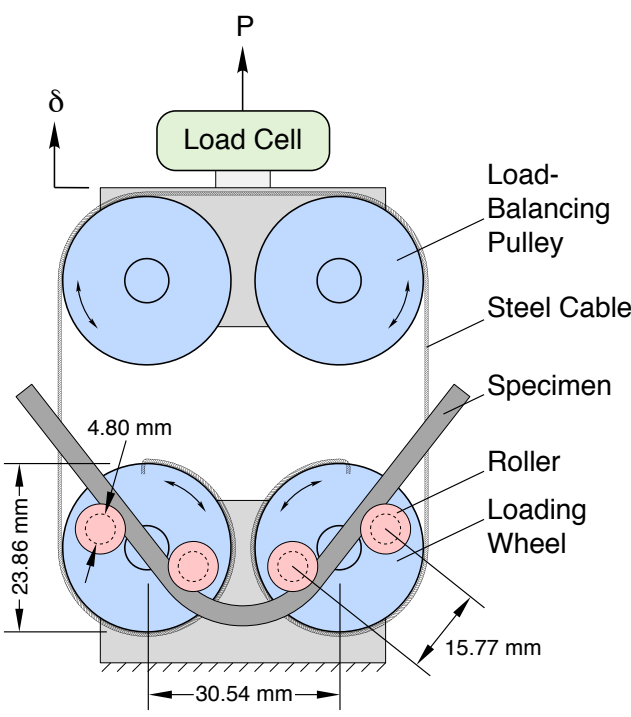

(b)

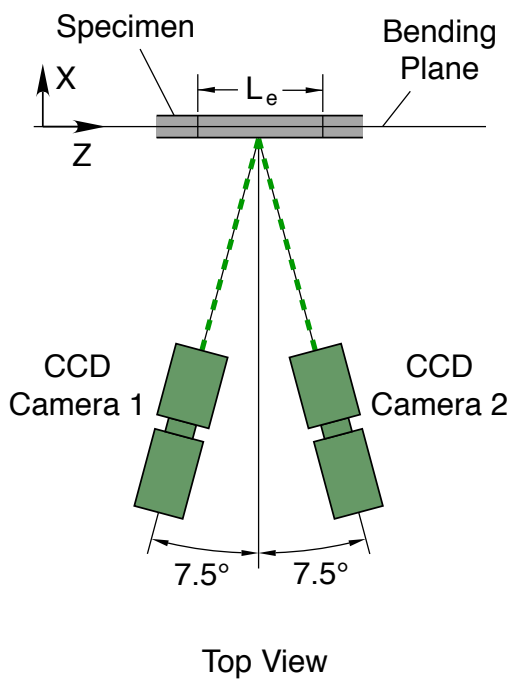

(c)

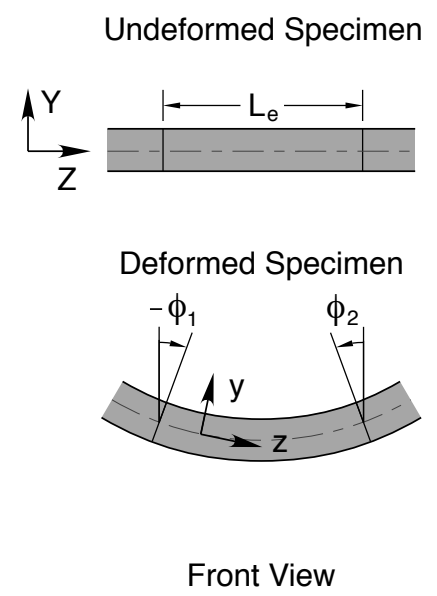

(d)

Figure 3: Bending setup: (a) photographs of overall setup and closeup of bending fixture with undeformed tube specimen, (b) schematic of pure-bending fixture with deformed specimen, (c) top view schematic, (d) front view schematics.

the foreground of the photographs in Fig. 2. The cameras were Pt. Grey Research Grasshoppers ${ }^{2}$ (GRAS50 S5M-C), each with a $2048 \times 2448$ pixel array of square $3.45 \mu \mathrm{m}$ pixels. For the uniaxial experiments the cameras had a stereo angle of $18^{\circ}$ between them. The cameras were attached with Fujinon HF75SA $75 \mathrm{~mm}$ focal length lenses with either $32.5 \mathrm{~mm}$ or $35 \mathrm{~mm}$ extension tubes. The aperture diameter of each lens was set to $9.4 \mathrm{~mm}$, which provided sufficient depth of field to keep both the crown and edge of the tube in focus. The exposure time ranged from $50.7 \mathrm{~ms}$ to $69.3 \mathrm{~ms}$ depending on the lighting. With the well known temperature sensitivity of $\mathrm{NiTi}$, special care was taken to choose lighting sources that did not affect the temperature of the specimen. We used fluorescent lights behind frosted translucent plastic, similar to a photographer's light box, to produce a diffuse, flat light (just out of view in Fig. 2(a) and 2(b), but just in view in Fig. 3(a)). Flexible fiber optic lights, as seen extending in from the bottom of the photos, with an adjustable light intensity were used to finely adjust the illumination and overcome shadows caused by the grips. Prior to the experiments, the DIC stereo system was calibrated using a $9 \times 9$ calibration grid with $1.34 \mathrm{~mm}$ spacing (Grid B, P/N AIG 045466 in Correlated Solutions (2010b)). During the experiment, a

\footnotetext{
${ }^{2}$ The manufacturer reports the Grasshopper is capable of 14 bit grayscale resolution, but the effective (noise free) dynamic range was approximately 8 bits for our setup.
} 
function generator triggered the cameras every 10 seconds, and the signal was sent to the data acquisition system to synchronize all measurements, including load, thermocouple, and crosshead displacement. After each experiment, the DIC images were analyzed using the commercially-available Vic-3D 2010 software (Correlated Solutions, 2010a) across a region of interest, centered on the tube axis (roughly 438 pixels across the outer diameter). We utilized a $21 \times 21$ subset, an analysis grid with $2 \times 2$ pixel spacing, and Vic-3D's default cross correlation function of the normalized sum of squared differences (Sutton et al., 2009). For the strain calculation at each analysis grid point, Vic-3D used a truncated Gaussian filter over a 31 pixel diameter circle with a weight of 1 at the center point and a weight of 0.1 at the circle edge.

The strain components from DIC analysis are reported with respect to a referential Cartesian coordinate system $(X, Y, Z)$ with $Z$ along the tube axis, or a cylindrical coordinate system $(R, \Theta, Z)^{3}$ aligned with the tube radius, circumference, and axis. We chose to report the Biot strain, instead of Vic-3D's default strain measure of Green-Lagrange strain. The Green-Lagrange strain is non-linear in the displacement in uniaxial tension, and the conjugate $2^{\text {nd }}$ Piola-Kirchhoff stress $\left(\boldsymbol{S}=\boldsymbol{N} \cdot \boldsymbol{F}^{-T}\right)$ has no simple physical interpretation. In particular, NiTi has a load plateau in uniaxial tension, where the force is constant with increasing elongation. However, $S_{Z Z}=\lambda_{Z Z}^{-1} P / A_{0}$ has a negative tangent modulus along this load plateau, since the global axial stretch ratio $\lambda_{Z Z}$ is a monotonically increasing quantity. The Biot strain tensor, on the other hand, is defined as

$$
\boldsymbol{E}^{\mathrm{B}} \equiv \boldsymbol{U}-\boldsymbol{I}
$$

where $\boldsymbol{U}$ is the right stretch tensor (from the polar decomposition of the deformation gradient $\boldsymbol{F}=\boldsymbol{R} \cdot \boldsymbol{U}$ with rigid rotation $\boldsymbol{R})$ and $\boldsymbol{I}$ is the identity tensor. The Biot strain $\left(\boldsymbol{E}^{\mathrm{B}}\right)$ and its conjugate Biot stress $\left(\boldsymbol{\Pi}^{\mathrm{B}}\right)$ are referential finite deformation measures that are convenient for the large rotation bending of slender structures, where the local direction of uniaxial loading follows the material rotation during the loading process. Accordingly, the rotation $\boldsymbol{R}$ can be applied to the reference coordinate system $(X, Y, Z)$ to define a new local, co-rotated, orthonormal frame $(x, y, z)$, as shown in Fig. 3(d). During bending, this local frame rotates about the $X$-axis to keep the $z$-axis aligned with the current beam axis and normal to the cross section (neglecting any shear deformation). Using our coordinates, the Biot strain $E_{Z Z}^{\mathrm{B}}$ coincides with the engineering strain in the co-rotated frame $\left(\lambda_{Z Z}-1\right)$, even when local strains and rotations are large. Likewise, the Biot stress component $\Pi_{Z Z}^{\mathrm{B}}$ is the engineering stress $\mathrm{d} P_{z} / \mathrm{d} A_{0}$, for an axial load $P_{z}$ and a reference cross-sectional area $A_{0}$ originally normal to the $Z$-direction. A detailed justification of this choice of stress and strain measures, and some further comments, are provided in Appendix B.

The stereo DIC setup for the bending experiment was similar to that of the tension and compression experiments. Since the bending fixture oriented the tube horizontally instead of vertically, the cameras were rotated $90^{\circ}$ about each of their optical axes and the stereo angle was reduced to $15^{\circ}$ to keep the length of the tube in focus. The DIC processing parameters described previously were used, but additional steps were taken as follows: (1) The coordinate system was carefully aligned so that the $X$-axis was precisely perpendicular to the bending plane and the $Z$-axis was aligned with the centerline of the tube specimen (see Fig. 3(c)). (2) The material curvature for an initially straight beam is defined as $\kappa=\mathrm{d} \phi / \mathrm{d} Z$, where $\phi$ is rotation of the cross section about the $-X$-axis, and $Z$ is the reference axial position. The average curvature $\bar{\kappa}$ over length $L_{\mathrm{e}}$ is $\left(\phi_{2}-\phi_{1}\right) / L_{\mathrm{e}}$, where $L_{\mathrm{e}}=Z_{2}-Z_{1}$ and $\phi_{i}$ are shown in Fig. 3(d). To measure $\phi_{i}$, we took two lines of constant $Z_{i}$, found their current positions $\left(y_{i}, z_{i}\right)$ over 438 points across the diameter, and used the slope of a least squares fitted line. (3) The position of the neutral axis, defined as $E_{Z Z}^{\mathrm{B}}=0$, was found for every set of data along $L_{\mathrm{e}}$ and then averaged over $Z$ to calculate the average neutral axis offset $\bar{Y}_{0}$.

\section{Tension and Compression Experiments}

The mechanical responses of the tube specimens were first characterized in uniaxial tension and compression at nearly isothermal elongation rates in room temperature air. Here, we present uniaxial experiments

\footnotetext{
${ }^{3}$ For most variables, upper case coordinates refer to reference coordinates, while lower case coordinates refer to current coordinates. Where certain symbols have already been defined, a subscript or superscript ' 0 ' is added to indicate a reference quantity.
} 
for later comparison to the room temperature bending response of the tube. A more comprehensive set of experiments on similar tubes at various temperatures and rates can be found in Churchill $(2010)^{4}$

The tubes studied herein exhibit a significant degree of tension-compression asymmetry, as is expected for textured NiTi. The global mechanical responses of the tube specimens in tension (experiment T1) and compression (experiment C1) are shown respectively in Figs. 4(a) and 4(b). The vertical axis is the Biot (engineering) stress $\bar{\Pi}_{Z Z}^{\mathrm{B}}=P / A_{0}$ averaged over the cross sectional area of the tube, and the horizontal axis $\bar{E}_{Z Z}^{\mathrm{B}}$ is the Biot strain along the tube axis, averaged over the points in the DIC "extensometer" gage length $L_{\mathrm{e}}$. The tensile response exhibits flat transformation plateaus (see (2)-6) in Fig. 4(a)), while the compressive response does not (see (4)-(6) in Fig. 4(b)). The magnitude of transformation strain in tension is larger than that for compression, and the magnitude of transformation stresses in tension are smaller than for compression. The details of the two responses and their differences are examined further in the following three subsections.

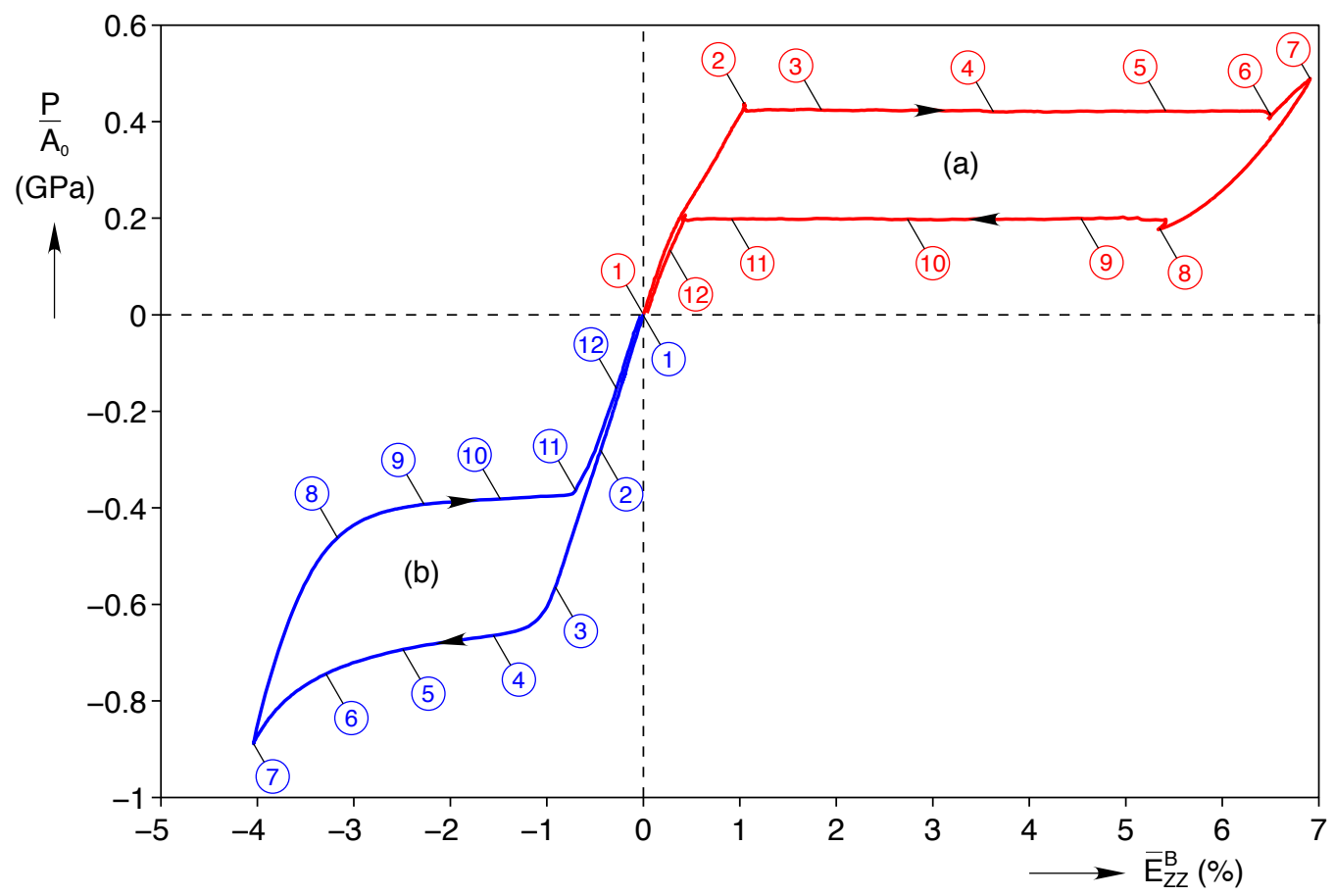

Figure 4: Mechanical responses of tube specimens during (a) tension experiment T1 and (b) compression experiment C1, showing significant tension-compression asymmetry. The circled numbers in (a) and (b) correspond to the strain field images in Fig. 5 and Fig. 7, respectively.

\subsection{Tension Results}

Here we describe the tensile mechanical response shown in Fig. 4(a). The initial slight non-linearity from $\bar{E}_{Z Z}^{\mathrm{B}}=0.2 \%$ to $0.6 \%$ is due to the rhombohedral phase (Miyazaki and Otsuka, 1986), which is commonly present in $\mathrm{Ni}$-rich $\mathrm{NiTi}$ in an intermediate range of temperatures and stresses. This is a relatively minor effect in the mechanical response, however, compared to the larger transformation to martensite. After the initially stiff segment, a distinct load plateau exists during loading (during $\mathrm{A} \rightarrow \mathrm{M}^{+}$transformation), and another lower plateau exists during unloading (during reverse, $\mathrm{M}^{+} \rightarrow \mathrm{A}$ transformation) $)^{5}$. Each plateau is associated with a material instability, accompanied by propagating necks separating regions of high and low strain. (See Section 2.4 of Chang et al. (2006) for a discussion of why this is indeed a material instability and not just a structural instability.) Axial strain field (DIC) contours of the front half of the tube specimen

\footnotetext{
${ }^{4}$ The tubes in Churchill (2010) are from the same manufacturer, with the same part number, as the tube specimens for this paper. The results are not shown here, however, because the tubes in Churchill (2010) are from an older lot (\# 358311), with different mechanical properties than the lot (\# 957152) studied here.

${ }^{5}$ Using a macroscopic viewpoint, we denote stress-induced tensile martensite as $\mathrm{M}^{+}$and stress-induced compressive martensite as $\mathrm{M}^{-}$
} 


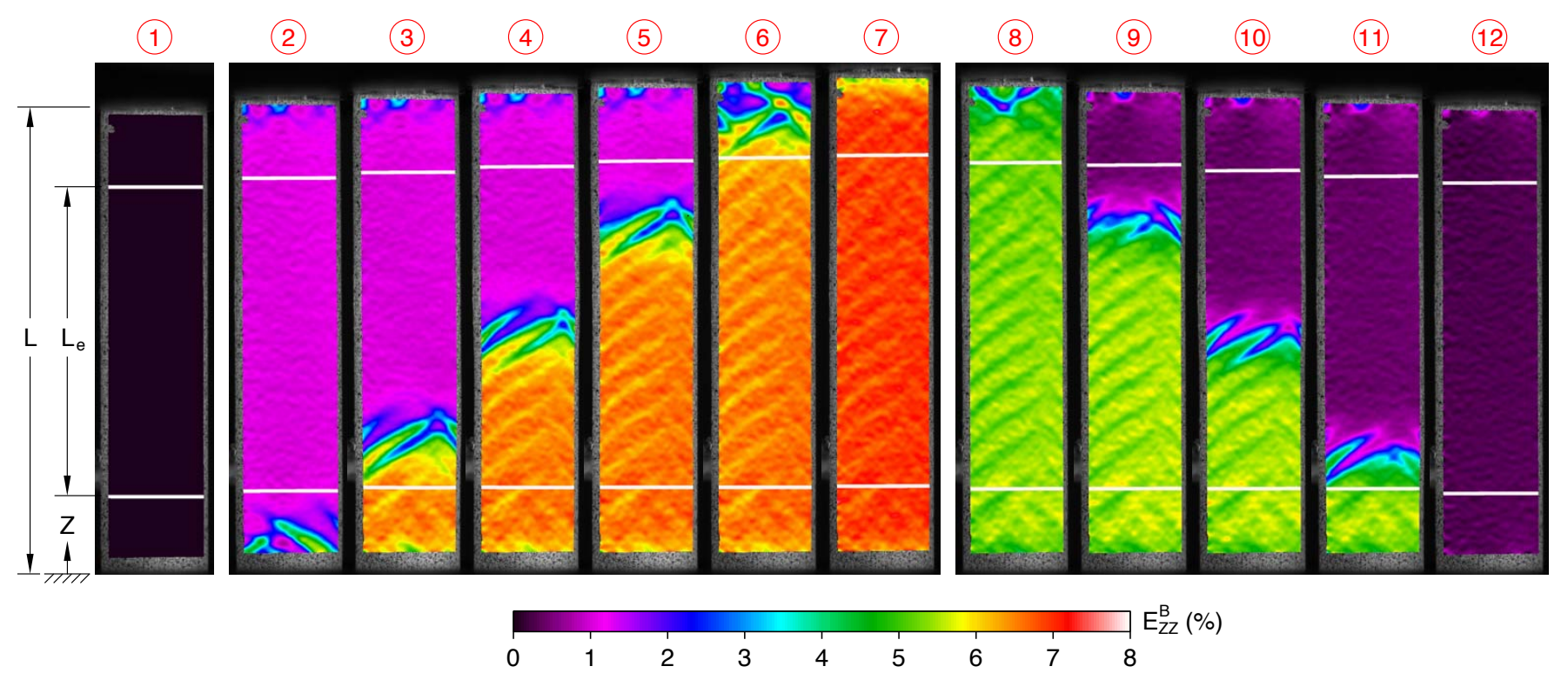

Figure 5: Experiment T1. Axial strain field images from DIC at times labeled in Fig. 4(a). The white lines in each image were superimposed to identify the limits of the DIC extensometer gage length. A macroscopic $\mathrm{A} \rightarrow \mathrm{M}^{+}$transformation front propagates from the bottom grip to the top grip in images (2) to (6) during loading, and a $\mathrm{M}^{+} \rightarrow \mathrm{A}$ front propagates downward in images (8) to (11) during unloading.

are shown in Fig. 5, and the circled numbers correspond to labeled points in Fig. 4(a). The two white lines in the strain field images identify the boundaries of the DIC extensometer gage length. Images (2)-6) clearly show a single macroscopic $\mathrm{A} \rightarrow \mathrm{M}^{+}$transformation front propagating from the lower grip to the upper grip during the loading plateau, and images (8)-(11) show a $\mathrm{M}^{+} \rightarrow \mathrm{A}$ transformation front propagating from the upper grip to the lower grip during the unloading plateau. Upon complete unloading, the average residual strain was quite small $\left(\bar{E}_{Z Z}^{\mathrm{B}}=0.03 \%\right)$, indicating nearly perfect superelasticity.

Examining Fig. 5 more closely, we see the macroscopic fronts are actually composed of fine "fingers" of high and low strain that convolute around the tube as the front propagates. The fingers appear slightly curved in Fig. 5, since they wrap around the curved surface of the tube. When the DIC data is "unwrapped" to a flat surface, as in Fig. 6(a), the fingers are nearly straight and their angle can be accurately measured. In Fig. 6(a) the trailing edge angle and leading edge angle of one of the fingers is $\alpha_{1}=57.2^{\circ}$ and $\alpha_{2}=64.1^{\circ}$, respectively, to the $Z$-axis. The fingers in four other images (not shown) were also measured and $\alpha_{1}$ ranged from $56.1^{\circ}$ to $58.5^{\circ}$, while $\alpha_{2}$ ranged from $64.1^{\circ}$ to $66.0^{\circ}$. Only the tips of the fingers on the right side of the tube are visible, making it difficult to measure their leading and trailing edges. In image (3) the finger on the right side protruded a little further into the DIC FOV allowing us to measure its trailing edge angle $\alpha_{3}=-59.6^{\circ}$. Since the unwrapped tube is similar to a thin strip, a simple Mohr's circle analysis similar to that shown in Shaw and Kyriakides (1998) gives an estimate for the ideal in-plane front angle $\alpha$ according to

$$
\cos (2 \alpha)=-\frac{1-\nu_{Z \Theta}}{1+\nu_{Z \Theta}}
$$

Using the DIC measured Poisson ratio $\nu_{Z \Theta}=-\bar{E}_{\Theta \Theta}^{\mathrm{B}} / \bar{E}_{Z Z}^{\mathrm{B}}=0.45$ (discussed further in section 4.3) results in $\alpha= \pm 56.1^{\circ}$, which is reasonably close to the angles measured.

The fine fingers at the austenite/martensite front interface are consistent with the observations of Feng and Sun (2006), who also studied superelastic NiTi tubes in tension. They explained that the branched finger morphology "helps to reduce the strain energy of the domain at the expense of increasing the total front length and therefore the total interfacial energy of the front." Put a different way, the material cannot suffer a jump discontinuity in lateral strain, so a macroscopic neck forms. Rather than a diffuse transition from low strain to high strain, fingers near the ideal angle form within the neck to minimize the strain energy associated with the incompatibility. Interestingly, Feng and Sun observed fingers that all pointed the same direction; whereas, the fingers on the left and right side of our tube have positive and negative angles, respectively, resulting in a criss-cross pattern. While Feng and Sun's loading rate was similar to ours $\left(\dot{\delta} / L= \pm 3 \times 10^{-5}\right.$ 


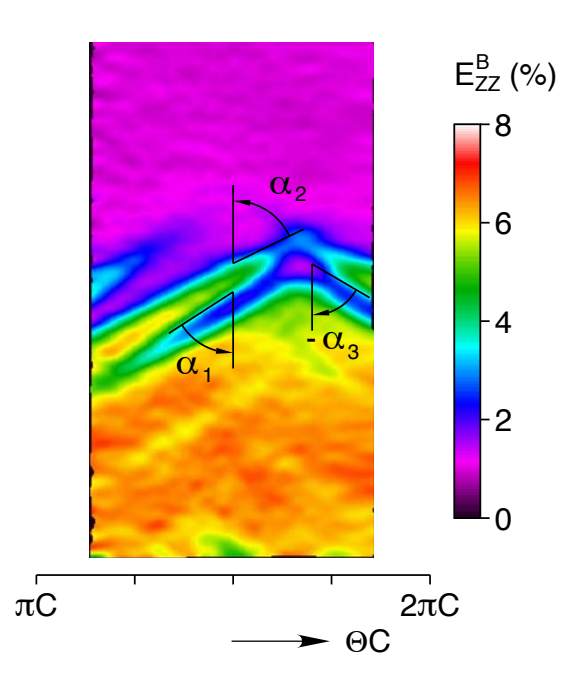

(a)
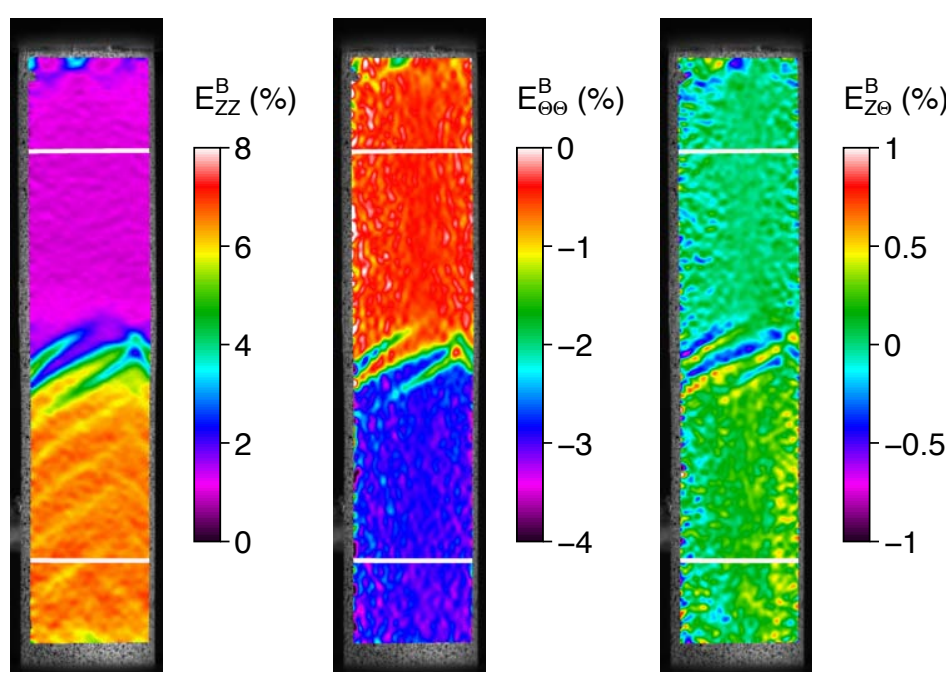

(b)

Figure 6: Experiment T1.(a) Magnified and unwrapped view of the "fingers" at time (3) of Fig. 5. (b) Surface strain fields (axial, hoop, and shear strain) from DIC at time (4).

$\mathrm{s}^{-1}$ ), the different observation may be due to different specimen shape (their dogbone versus our straight specimens), different onset of front propagation (their $M$ first nucleated as a helix, then transformed into the finger morphology), different gage section dimensions (their $D / H=13.3$ and $L / D=23$, while our $D / H=10$ and $L / D=4.45$ ), different grips, and/or different load stack torsional rigidities. Other tension experiments on thin superelastic strips (Shaw and Kyriakides, 1997, 1998) have also showed similar crisscrossing fingers. Shaw and Kyriakides found that the occurrence of criss-crossing morphology was sensitive to the specimen length and the elongation rate, being more prevalent for short specimens (to minimize bending misalignment with the tensile axis caused by the small axial kink across angled fronts) and very slow elongation rates (to minimize temperature gradients during exothermic/endothermic transformations). However, we performed another experiment, not shown here, on a longer tube specimen $L=56.30 \mathrm{~mm}$ $(L / D=17.7)$ which resulted in similar crisscrossing. More data is necessary to determine exactly what scenarios cause or inhibit crisscrossing, but this is left for future work since it does not impact the current study.

Before discussing the compression results, we make a few comments about our choice of DIC extensometer gage length and grip effects in the tensile results. Close inspection of image (7) and (12) reveals that the region affected by the grip is about $D / 4$ long when the strain fields are mostly uniform. On the other hand, the boundary effect protrudes about $3 D / 4$ into the gage length when a front is near the grips. This can be seen in the altered front morphology in images (2), (6), and (8), compared to (3)-(5) and (9)-(11) where the front is far from the grips. Incidentally, DIC allowed us to select our extensometer gage length after the experiment rather than before, so the two white lines in image (1) were selected as the boundaries of $L_{\mathrm{e}}$ to avoid these grip effects. Also note that the proximity of the front to the grips (images (2), (6), and (8) is reflected in the axial load in Fig 4(a). The small increases and drops in load at the beginning and end of the load plateaus correspond to when the front emerged from or entered the grips. Stress concentrations at the grips tend to suppress these load peaks, unless care is taken to suppress transformation at the grips (Iadicola and Shaw, 2002b; Feng and Sun, 2006), but in this case gripping was uniform enough that small peaks were still evident.

DIC provides more than just the axial strain component, so it is interesting to see for the first time some of the other in-plane strain components to more fully characterize details of the localized strain field. As an example, Fig. 6(b) provides a snapshot of the tube surface strain field with respect to axial, hoop, and (tensorial) shear strain components (from left to right $E_{Z Z}^{\mathrm{B}}, E_{\Theta \Theta}^{\mathrm{B}}$, and $E_{Z \Theta}^{\mathrm{B}}$ ) at time (4). Note the change in color scale for each strain component. The front fingers are apparent at the middle of the specimen length in all three strain components, most clearly evident in the axial and hoop strains and somewhat less distinct in the shear strain. The hoop strain is like a reverse video image of the axial strain taken to negative strains. 
The hoop strains in the upper and lower regions where the strain is nearly uniform allows the Poisson ratio to be measured as previously mentioned $\left(\nu_{Z \Theta}=0.45\right)$. The shear strain fluctuates between small positive and negative values across each front finger (about $\pm 0.4 \%$ ). The angled striations in the axial strain field seen in the wake of the front (lower half, $\mathrm{M}$ region), however, are absent in the hoop strain and shear strain. The shear strain field image shows a small positive shear strain of between $0.1 \%$ to $0.4 \%$ in the lower half (M region).

\subsection{Compression Results}

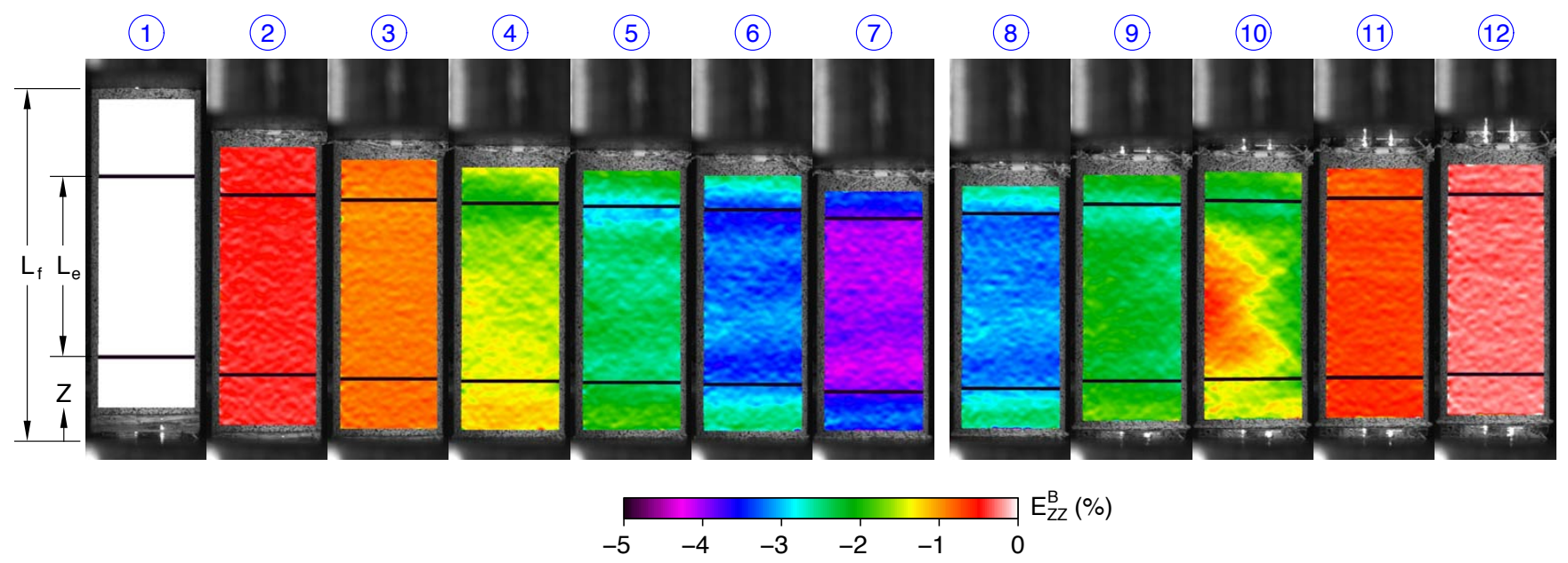

Figure 7: Experiment C1. Compressive axial strain field contours at times labeled in Fig. 4(b). Unlike the tensile strain field images of Fig. 5, the strain fields in compression are relatively uniform, except for some transient bending in image (10).

Using DIC, accurate measurements of the uniaxial compression response of a tube specimen and the corresponding strain field contours were obtained and are shown in Figure 4(b) and Fig. 7, respectively. High quality compression experiments of SMAs are difficult to achieve, and are much less prevalent in the literature than tension experiments. In this case, DIC strain measurement was essential to successfully obtain accurate measurements. Recall that $\bar{E}_{Z Z}^{\mathrm{B}}$ is the axial strain from the DIC analysis averaged over the chosen gage length $L_{\mathrm{e}}$ and not the grip strain $\delta / L$. The grip strain measurement was contaminated by the specimen settling into the grips, grip compliance, and a small friction between the specimen and the grips. Even with careful alignment and attempts to avoid specimen settling by a pre-test elastic load-unload, the grip strain and $\bar{E}_{Z Z}^{\mathrm{B}}$ differed by as much as $1.8 \%$ strain.

While distinct transformation fronts in SMAs have been observed in tension using full field techniques, no such techniques have been used in compression to our knowledge. Due to the lack of a distinct stress plateau during isothermal $\mathrm{A} \rightarrow \mathrm{M}^{-}$transformation, one expects that transformation fronts are not present in compression, and this is generally confirmed in the strain field images of Fig. 7, with one exception discussed below. The strain fields are nearly homogeneous during loading (images (1)-(7)), aside from minor strain perturbations near the grips and minor axial strain fluctuations. The reason why tension exhibits strain localization, while compression does not, has been a long-standing open scientific question. Mao et al. (2010), however, recently provided a plausible explanation. Using electron backscatter diffraction on NiTi tubes, they found that grains favorably aligned for tension had a distribution of Schmid factors within $\pm 8.7 \%$, while the grains favorably aligned for compression varied by $\pm 20 \%$. They argued that a narrow distribution of Schmid factors leads to an auto-catalytic effect, where transformation in one grain causes neighboring grains to transform, leading to a macroscopic front. Conversely, a large distribution of Schmid factors means that transformation is less likely to spread to neighboring grains without a large increase in stress, and this leads to a more diffuse transformation involving non-adjacent grains.

Returning to our compression experiment, it appears that the tube buckled during mid $\mathrm{M}^{-} \rightarrow \mathrm{A}$ (reverse) transformation, which is an unexpected result and to our knowledge has never been reported before. During unloading from compression (images (8)-(12)), the strain field is nearly homogeneous except for image (10) where the tube is slightly bent. The strain varies from $-0.7 \%$ to $-1.9 \%$ from the left to the right side 
of the specimen. The adjacent strain fields in images (9) and (11), however, are quite uniform, indicating that buckling is only a transient phenomenon. More precisely, we defined the tube as "buckled" when the difference in axial strain between left and right sides of the tube exceeded $0.375 \%$ strain, using the full set of DIC data (not shown). Buckling occurred between $\bar{E}_{Z Z}^{\mathrm{B}}=-2.2 \%$ and $-0.8 \%$, which corresponded to when the average tangent modulus, $\mathrm{d} \bar{\Pi}_{Z Z}^{\mathrm{B}} / \mathrm{d} \bar{E}_{Z Z}^{\mathrm{B}}$, dropped below $2 \mathrm{GPa}$ in this case. At image (10) the average tangent modulus is only $1.1 \mathrm{GPa}$. Buckling is influenced by material properties, specimen geometry, and boundary conditions, so the $2 \mathrm{GPa}$ threshold only applies to our specific experimental setup. The origin of this buckling phenomenon is difficult to isolate, but we believe residual stresses induced during the $\mathrm{A} \rightarrow \mathrm{M}^{-}$ transformation (loading) delayed the onset of $\mathrm{M}^{-} \rightarrow \mathrm{A}$ transformation during unloading until the tangent modulus reached a sufficiently low value to trigger a structural instability. Nevertheless, the specimen reverted to a straight shape, and the final residual strain at zero load was quite small, $\bar{E}_{Z Z}^{\mathrm{B}}=-0.03 \%$.

\subsection{Comparison of Tension E3 Compression Responses}

The tension and compression behavior are directly compared in Fig. 8(a), using absolute values of stress and strain. The lower portion of the plot shows two pairs of mechanical responses for tension and compression, respectively, with the previously presented tension/compression experiments (T1 and C1) shown by dotted lines and second tension/compression experiments (T2 and C2) shown by solid lines. The upper portion of the plot, using the vertical axis on the right, shows the magnitude of the average hoop strain, $\bar{E}_{\Theta \Theta}^{\mathrm{B}}$, as a function of the average axial strain magnitude during the second set of experiments (T2 and $\mathrm{C} 2$ only). The second tension experiment and second compression experiment were performed (on separate new specimens) to characterize the behavior at larger strains, consistent with the bending experiments of the next section.

One can see that the respective pairs of tension and compression responses agree quite well during the loading segments. There was one small deviation during the second tension experiment. Two fronts, rather than one, were observed during the $\mathrm{A} \rightarrow \mathrm{M}^{+}$transformation, which caused the small dip in stress between $\bar{E}_{Z Z}^{\mathrm{B}}=5.5 \%$ and $6.5 \%$ when the fronts neared one another and then coalesced. The first and second compression experiments, on the other hand, had similar uniform strain fields during loading.

The unloading responses of the second set of experiments are clearly different, since unloading commenced at much larger stresses. In the second tension experiment, the loop was not closed. A residual strain of $7.49 \%$ indicates the occurrence of significant plastic (slip) deformation and likely significant "locked-in" martensite (although we did not heat the specimen to measure how much could be ultimately be recovered). The second compressive response, however, had only $-0.09 \%$ residual strain after unloading, even after being subjected to a stress of $-1.46 \mathrm{GPa}$ ! Nonetheless, comparing the unloading curve to the first compression experiment does show some signs of the extreme compressive stress during loading. First, the unloading $\mathrm{M}^{-} \rightarrow \mathrm{A}$ transformation stress was about $37 \mathrm{MPa}$ lower (at $-1.74 \%$ strain), indicating that a larger reverse driving force was needed to overcome locked-in martensite. Second, the transient buckling instability in the axial strain field was observed again when the tangent modulus dipped below $2 \mathrm{GPa}$, but this occurred between $\bar{E}_{Z Z}^{\mathrm{B}}=-2.8 \%$ and $-1.0 \%$, which is a $28 \%$ larger span than the first experiment. The increase supports our earlier hypothesis that the large compressive stresses during loading play a role in the buckling instability during unloading.

Since DIC provides all surface strain components, we were also able to examine the development of the lateral (hoop) strain $E_{\Theta \Theta}^{\mathrm{B}}$. The upper plot of Fig. 8(a) provides the magnitude of the hoop strain averaged across the gage length $L_{\mathrm{e}}\left(\left|\bar{E}_{\Theta \Theta}^{\mathrm{B}}\right|\right.$, right hand vertical axis) against the magnitude of the average axial strain $\left|\bar{E}_{Z Z}^{\mathrm{B}}\right|$ (horizontal axis) for the second set of tension/compression experiments (T2 and $\mathrm{C} 2$ ). In the small strain limit, the ratio $\nu_{Z \Theta} \equiv-E_{\Theta \Theta}^{\mathrm{B}} / E_{Z Z}^{\mathrm{B}}$ is the Poisson's ratio, so the slope of the response curve $\left|\bar{E}_{\Theta \Theta}^{\mathrm{B}}\right|$ versus $\left|\bar{E}_{Z Z}^{\mathrm{B}}\right|$ gives an approximate Poisson's ratio, presuming the tube specimen is transversely isotropic about its centerline. The average lateral strain data from the two sets of experiments ( $\mathrm{T} 1$ and $\mathrm{T} 2$; $\mathrm{C} 1$ and C2) overlapped well, so only experiments T2 and C2 are shown here. The initial slope in experiment T2 is 0.45 up to about $\bar{E}_{Z Z}^{\mathrm{B}}=7.9 \%$, where the slope changes to 0.39 as transformation is accompanied by significant plastic (slip) deformation. The compression experiment $\mathrm{C} 2$ has a slope of 0.45 until $\left|\bar{E}_{Z Z}^{\mathrm{B}}\right|=1.1 \%$, where the slope changes to 0.51. A hysteresis in the Poisson's ratio exists between loading and unloading in both tension and compression (most noticeable in $\mathrm{T} 2$ ), since linear elastic deformation likely dominates the response during unloading, but the effect is rather small. 

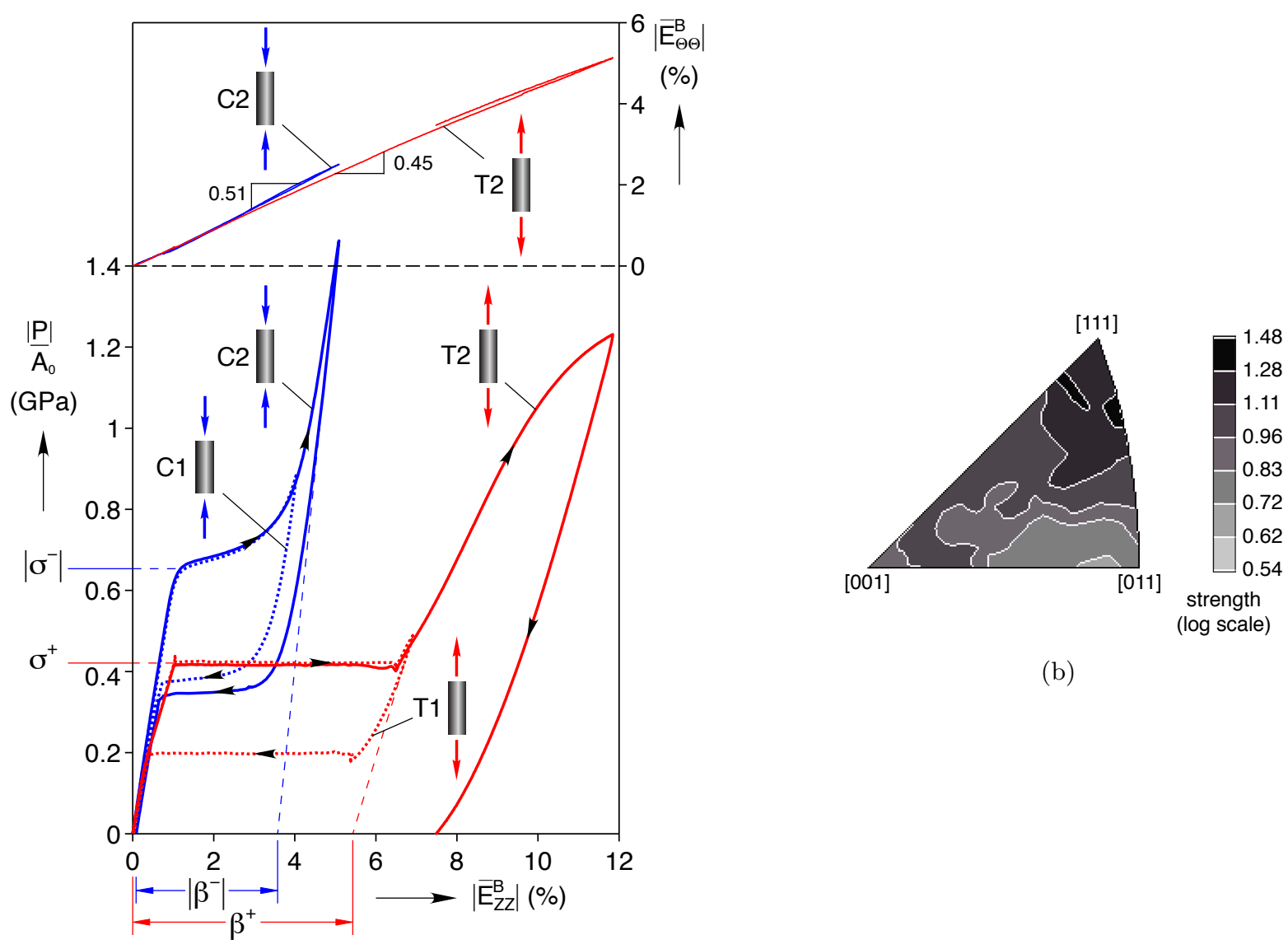

(b)

(a)

Figure 8: Comparison of tension and compression responses: (a) Lower plot shows four mechanical responses in tension (experiments T1 and T2) and compression (experiments C1 and C2) with magnitudes of transformation stresses and strains labeled. Upper plot shows the magnitude of the lateral (hoop) strain against the axial strain for tension experiment T2 and a compression experiment C2, with slopes indicating the measured Poisson ratios. (b) X-ray contour map, showing significant $\langle 111\rangle_{\mathrm{B} 2}$ texture along the drawing axis of a similar tube (provided courtesy of K. Gall, J. Tyber, and H. Maier).

While the tension-compression asymmetry of drawn NiTi has been shown before, compression results are rare in the published literature, and a quantitative comparison is worthwhile. Initially the tensile and compressive linear elastic moduli match almost perfectly $\left(E_{A}=65.3 \mathrm{GPa}\right)$, as one would expect from simple elastic deformation of the austenite lattice. After $\left|\bar{E}_{Z Z}^{\mathrm{B}}\right|=0.2 \%$ the tensile and compression responses start to deviate, since the small R-phase non-linearity in tension is absent in compression. As the strain exceeds $\left|\bar{E}_{Z Z}^{\mathrm{B}}\right|=1 \%$, the tension/compression asymmetry becomes more pronounced. The $\mathrm{A} \rightarrow \mathrm{M}^{+}$transformation (plateau) stress in tension is $\sigma^{+}=421 \mathrm{MPa}$, and the $\mathrm{A} \rightarrow \mathrm{M}^{-}$"plateau" in compression is $\sigma^{-}=-654 \mathrm{MPa}$ at its onset. Transformation strains were estimated by projecting a linear elastic tangent from the initial unloading response to the zero stress axis as shown in Fig. 8(a), and then subtracting the small amount of final residual strain. In tension the transformation strain is $\beta^{+}=5.43-0.03=5.40 \%$ (incidentally, quite close to the length of the plateau, $5.45 \%$ strain) and in compression is about $\beta^{-}=-3.58+0.09=$ $-3.49 \%$. Using a simple Gibbs free energy model (see Appendix $\mathrm{C}$ ), the isothermal driving force for $\mathrm{A} \rightarrow \mathrm{M}$ transformation is linear in the quantity $\sigma \beta$. The model predicts transformation to occur at a constant stress, which is reasonable in light of our tension responses, but less so for our compression responses without true plateaus. Nevertheless, the model predicts that the ratio of tension to compression, $\sigma^{+} \beta^{+} /\left(\sigma^{-} \beta^{-}\right)$, should be near unity for experiments at the same temperature. Based on our measurements, the ratio is 0.996 which is consistent with basic thermodynamic theory.

The tension-compression asymmetry is generally attributed to the crystallographic asymmetry of the martensitic phase transformation. The tube drawing process typically creates a prevalent $\langle 111\rangle_{\mathrm{B} 2}$ texture of the austenite lattice aligned with the longitudinal tube axis. This was confirmed by X-ray diffraction measurements performed on an as-received NiTi tube specimen in collaboration with K. Gall, J. Tyber, 
and H. Maier. Three pole figures $\left(\langle 110\rangle_{\mathrm{B} 2},\langle 100\rangle_{\mathrm{B} 2}\right.$ and $\left.\langle 211\rangle_{\mathrm{B} 2}\right)$ were collected from the tube cross-section (surface with normal along the longitudinal tube axis), and the inverse pole figure shown in Fig. 8(b) was calculated from the three pole figures using the popLA software package (Kocks et al., 1995). The intensity contours in the stereographic triangle represent the density of poles aligned with the longitudinal tube axis, normalized by the pole density for a perfectly random orientation of poles. ${ }^{6}$ The $\langle 111\rangle_{\mathrm{B} 2}$ texture allows us to approximate the tube as a single crystal to estimate the transformation strains in tension and compression. Many researchers use the set of observed 24 habit plane variants (HPV) of martensite (Matsumoto et al., 1987) to model the superelastic behavior of SMAs (e.g. Patoor et al., 1996; Gall et al., 1999; Gao et al., 2000). Using this theory and the experimental values of Matsumoto et al. (1987), we calculate the uniaxial Biot strains along $\langle 111\rangle_{\mathrm{B} 2}$ to be $5.47 \%$ in tension and $-3.71 \%$ in compression, which agree reasonably well with our measured values of $5.40 \%$ and $-3.49 \%$, respectively. Details of the calculation are provided in Appendix D.

\section{Bending Experiments}

In this section, we present two bending experiments (B1 and B2) in room temperature air, each performed on new superelastic tube specimens cut from the same manufacturing lot used in the tension and compression experiments. The setup used 4-point (pure) bending as described in Section 3.2, and specimen dimensions were given in Table 1 . The end-rotations were controlled at constant, slow rates in both experiments.

Before presenting the experimental bending results and analysis, the implications of the significant tension-compression asymmetry exhibited by our NiTi tubes should be discussed. Not only is the magnitude of the transformation stress larger in compression, but the strain localizes in tension, while in compression it does not. Both observations have implications for bending:

- The neutral strain axis may deviate from the tube centerline. As depicted in Fig. 9, we define the neutral axis $\left(Y_{0}\right)$ as the location where the local axial strain is zero, $E_{Z Z}^{\mathrm{B}}\left(X, Y_{0}, Z\right)=0$. In pure bending the specimen has a zero net axial load. For conventional engineering materials that have symmetric tension-compression responses, the neutral axis remains at the centroid of the cross section. However, for our NiTi tubes, the neutral axis must shift towards the compressive side to balance the distribution of tensile and compressive stresses in the cross section. This effect has been predicted by several SMA beam models (e.g. De la Flor et al., 2010). Experimentally, Rejzner et al. (2002) inferred the neutral axis position using strain gages on the top and bottom of a SMA beam, but here DIC allows $Y_{0}$ to be measured directly for the first time.

- Plane-sections may not remain plane. Under the classical Euler-Bernoulli beam theory assumption, the strain distribution across the diameter is $E_{Z Z}^{\mathrm{B}}=-\kappa\left(Y-Y_{0}\right)$, where $\kappa \equiv \mathrm{d} \phi / \mathrm{d} Z$ is the referential beam curvature (as opposed to the strictly geometric curvature $\mathrm{d} \phi / \mathrm{d} z$ ), and $Y$ is the distance from the centroid in the reference (straight) configuration. Note that the Biot strain gives a distribution that is strictly linear in $Y$, regardless of the magnitude of $\kappa$, unlike the Green-Lagrange strain that is quadratic (Irschik and Gerstmayr, 2009). This strain distribution is shown as the kinematically preferred strain profile in Fig. 9(a). However, the fact that transformation tends to localize in tension and not in compression suggests a different strain distribution, more like that of Fig. 9(b). Intermediate tensile strains along the stress plateaus are associated with unstable material states (if otherwise unconstrained) which are largely absent in Fig. 5. The more compatible, kinematically preferred strain profile, however, requires strains in this unstable range. Thus, a competition exists between compatibility and local material stability, and one would expect an actual strain profile somewhere between the two extremes shown.

\footnotetext{
${ }^{6}$ The X-ray data shown was obtained on a tube specimen from the same manufacturer (Memry) of the same geometry, but from an older lot (\# 358311, used in Churchill (2010)) than the current lot (\# 957152, used in all experiments in this paper). Some differences exist in the mechanical responses between the two lots, such that tubes from the older lot had a $18 \%$ shorter $\mathrm{A} \rightarrow \mathrm{M}^{+}$plateau than the current lot. Based on this mechanical comparison, the strength of the $\langle 111\rangle_{\mathrm{B} 2}$ texture may be, if anything, under-predicted by Fig. 8(b) for the current lot.
} 
Kinematically Preferred

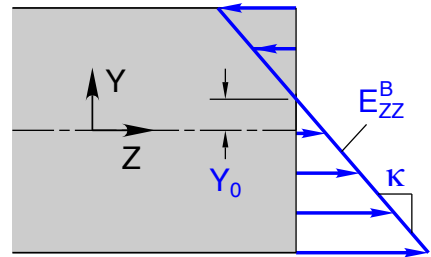

(a)
Material Preferred

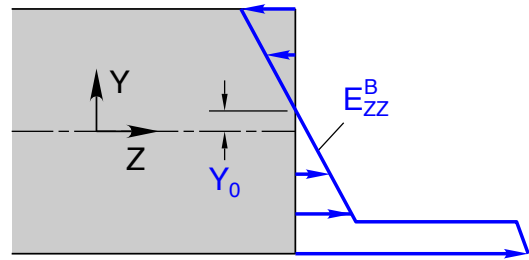

(b)

Figure 9: Schematics of two competing bending strain profiles.

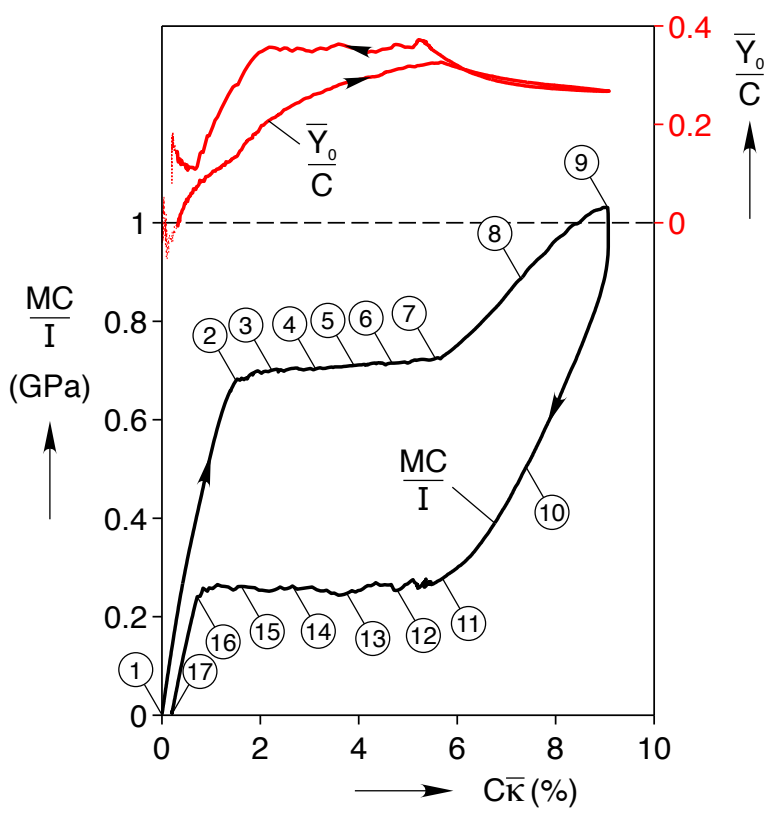

Figure 10: Experiment B1. Bending moment response (lower plot) and neutral axis (upper plot) versus average curvature for a single cycle. The circled images correspond to the strain field images in Fig. 11 and 12. As expected from the tension and compression responses, the moment-average curvature response shows a stiff-compliant-stiff progression during loading and unloading. Also, the neutral axis position of the bent tube deviates significantly from the centroid of the cross-section.

Here we measure full-field bending strain profiles in NiTi for the first time and observe how the material instability manifests in the presence of a significant lateral strain gradient. We first describe a single-cycle bend-unbend experiment (B1) taken to outer-fiber bending strains beyond the $\mathrm{A} \rightarrow \mathrm{M}^{+}$and $\mathrm{A} \rightarrow \mathrm{M}^{-}$uniaxial plateaus. Then, experiment B1 is compared to potential simple bending models, which are based on the results of the tension and compression loading responses. The section ends with a description of the second bending experiment (B2), an incremental 5-cycle experiment with progressively larger rotation amplitudes within the range used for experiment B1.

\subsection{Single-cycle Bending Experiment (B1)}

The global bending response of the tube during a single load-unload cycle is shown in Fig. 10 (lower plot), along with the average position of the neutral axis (upper plot). The lower left vertical axis is the normalized moment $M C / I$, where $M$ is the applied moment about the $x$-axis, $I$ is the corresponding area moment of inertia of the cross section, and $C=D / 2$ is the outer radius of the tube. The horizontal axis is the dimensionless curvature $C \bar{\kappa}$ averaged across the axial gage length $\left(L_{\mathrm{e}}=9.58 \mathrm{~mm}\right)$ from the DIC data. The axes, $M C / I$ and $C \bar{\kappa}$, are the classical formulas for the stress and strain at the outer fiber of a homogeneous, symmetric cross section, linear elastic beam with centroidal neutral axis. Berg (1995a) tested multiple wire diameters and found that these normalized quantities adequately remove the effect of geometry, despite the complex behavior of NiTi. The upper right vertical axis $\left(\bar{Y}_{0} / C\right)$ in Fig. 10 is the normalized reference position of the neutral axis $Y_{0}$ averaged over $L_{\mathrm{e}}$. The average neutral axis position at 
small curvatures is finely dashed with a lighter line weight, since the strains were too small to accurately measure $\bar{Y}_{0}$.

The circled labels in Fig. 10 correspond to the strain fields shown in Fig. 11 and 12 for bending (loading) and unbending (unloading), respectively. Note that the color scale is discontinuous at zero strain to clearly highlight the neutral axis in the strain field. The scallops in the strain field images at the top left and right (see Fig. 11) are dataless regions due to the rollers creating shadows on the specimen or obscuring the view of one of the DIC cameras. The thick black lines on the strain fields images of Fig. 11 and 12 are the two positions $\left(Z_{1}\right.$ and $\left.Z_{2}\right)$ where cross-section rotations $\left(\phi_{1}\right.$ and $\left.\phi_{2}\right)$ were determined and then used to calculate the average curvature. The red and green lines on the strain field images in Fig. 11(a) correspond to the strain profiles shown in Fig. 11(b). The black, red, and green lines appear curved because the strain fields and the lines were overlaid on images taken by camera 1 in Fig. 3(c) at $7.5^{\circ}$ to the bending plane. Viewed normal to the bending plane, these lines are straight, as can be seen later, for example, in Fig. 13(a). Note that the strain field images are overlaid on the deformed images of the tube by transforming to current coordinates $(x, y$, and $z)$ in Fig. 11(a), while the strain profiles are plotted with respect to reference coordinates $(Y / C)$ in Fig. 11(b). The strain profile predicted by classical Euler-Bernoulli beam theory is

$$
E_{Z Z}^{\mathrm{B}}(Y)=-\bar{\kappa}\left(Y-\bar{Y}_{0}\right),
$$

based on the measured average neutral axis $\left(\bar{Y}_{0}\right)$ and average curvature $(\bar{\kappa})$. These are shown by blue, dashed lines in Fig. 11(b) for comparison to the measured local strain profiles.

Similar to the uniaxial responses, the moment-curvature response in Fig. 10 during loading has three regimes: an initially stiff segment (1)-(2), a more compliant ("plateau") region with a small positive tangent modulus (2)-(7)), and a post-plateau stiff segment (7)-9). Referring to Figs. 10 and 11 and stepping through the loading process in detail:

- (1)-(2) The initial bending response is nearly linear, and as expected, the strain profile is linear. The average neutral axis starts near the origin, but by (2) has shifted upward (toward the compression side) to about $\bar{Y}_{0}=0.13 C$, likely due to the $R$-phase nonlinearity on the tension side.

- (2)-(3) The global tangent modulus undergoes an abrupt reduction at (2), and six very small moment drops (upon close inspection) occur soon thereafter in the moment-curvature response that coincide with the six strain localizations seen in image (3). The green line intersects one of these fingers, so the green line's strain profile is non-linear on the tension side of the tube. Thus, plane sections clearly do not remain plane. The red line does not cut through one of the fingers, but it also deviates from $-\bar{\kappa}\left(Y-\bar{Y}_{0}\right)$, albeit in the opposite direction. This indicates a varying transformation rate at each axial location along the tension side. Close inspection at (2) also reveals a kink in the evolution of the average neutral axis, after which it accelerates somewhat.

- (4)-(5) Further localizations appear, and two wedge-shaped regions develop on the tension side as localized strain regions coalesce. The average neutral axis position continues to shift upward, although the local neutral axis position has slight fluctuations near the apex of the wedge regions. Two, somewhat more diffuse, wedge shaped regions also appear on the compression side opposite the tension wedge regions. The green and red strain profiles deviate further from the blue, dashed line.

- (6) A third wedge forms on the tension side at the left, and the apex of the wedges have been driven nearly to the neutral axis. The red strain profile has a significant region of nearly constant $1.6 \%$ strain $(-0.7<Y / C<-0.1)$, after which it begins to accelerate back towards the linear, blue, dashed line.

- (7) The tube nearly reaches the end of the compliant region in Fig. 10. Accordingly, only a small region of low strain remains at $Y=-C$, next to the righthand black line in image (7). At this point the average neutral axis is shifted upward to $\bar{Y}_{0}=0.32 C$.

- (7)-(9) Soon after (7) the last region of low strain at $Y=-C$ disappears, and the moment response stiffens. By (8) the outer fiber strain on the tension side exceeds $8 \%$, and while the strain is still quite inhomogeneous, additional fingers have developed to fill in regions between the wedges. During (7) to 
(9) the average neutral axis has retreated somewhat toward the tube centerline. By (9) the average neutral axis reaches $\bar{Y}_{0}=0.27 C$, and the green and red strain profiles have nearly converged to the blue, dashed line. One can also see that the tube gage length has shifted to the left in the strain field image (9), since the rollers allow additional tube length to be drawn in (from the right in this case), but this does not adversely affect the quality of the data since DIC provides local strain and curvature measurement.

The response during unloading is similar to that of loading, but exhibits a large moment hysteresis and events generally occur in reverse order. The global moment-curvature response Fig. 10 also has three regions: a steep segment between (9) and (11), and serrated plateau between (11) and (16), and a final nearly linear unload segment after (16). Referring to Figs. 10 and 12 and stepping through the unloading process in detail:

- (9)-(11) Just after the end of loading, point (9), the end-rotations are briefly held fixed, and the normalized moment $(M C / I)$ relaxes from 1.03 to $0.926 \mathrm{GPa}$ as shown by the vertical segment in the momentcurvature response of Fig. 10. No change in the strain field is observed during this time. Soon after unloading commences, the moment-curvature response decreases in a monotonic manner with a shallowing tangent modulus. During (9)-(11), the strain field magnitudes decreases in a monotonic way, but otherwise the morphology remains relatively static. The average neutral axis position moves back upwards, retracing the path of the post-plateau loading response.

- (11)-(16) Shortly after (11) the unloading plateau begins in the moment-curvature response, and the average neutral axis position rises to a maximum (of the entire experiment) of $\bar{Y}_{0}=0.37 C$. The moment plateau during unloading does not have a positive tangent modulus (as it generally did during loading), and it is accompanied by a more jagged response than occurred during loading (2) to (7)). The fluctuations in $M C / I$ coincide with the disappearance of strain localizations in Fig. 12, and these happen in the reverse order that they were generated during loading. Although it may not be clear from the still images, a video of the DIC strain sequence shows that localizations vanish more abruptly during unloading than they had appeared during loading. The average neutral axis position remains relatively constant near $\bar{Y}_{0}=0.35 \mathrm{C}$ for most of the unloading plateau (unlike the monotonic increase during the loading plateau), at least until between (14) and (15) when it decreased steeply to $\bar{Y}_{0}=0.11 C$ at (16). This time interval, from (14) to (16), corresponds to the disappearance of the final localized wedge regions in the strain field.

- 16-17) The final stage of unloading occurs as a steep linear segment of the moment-curvature response. The final residual curvature at zero moment is $C \bar{\kappa}=0.19 \%$. The fully unloaded strain image (17) shows a small residual lateral strain gradient with maximum outer fiber strains of about $\pm 0.3 \%$.

Clearly, the strain localizations on the tension side and the absence of strain localization on the compression side are consistent with the unstable pure tension and stable (during loading) pure compression behavior shown in Fig. 5 and 7, respectively. The fingers look remarkably similar to those observed in uniaxial tension, and the wedges are an agglomeration of broadened fingers. Interestingly, the wedges on the tensile side bend the tube so far that it becomes kinematically necessary to form more diffuse wedges on the stable compression side as well. Close inspection of the raw images of the tube, as well as the three dimensional surface of the tube from DIC analysis, however, did not reveal any localized wrinkling on the compression side.

While the localizations in tension and bending are qualitatively similar, there are quantitative differences. First, recall that finger angles in uniaxial tension ranged from $56.1^{\circ}$ to $66^{\circ}$ across 5 different images. For this bending experiment, using an unwrapped version of image (5), the low strain-high strain interfaces ranged from $44.5^{\circ}$ to $70.5^{\circ}$ to the tube axis. The center of this range is still close to our estimate of $56.1^{\circ}$ for the ideal angle, but the spread is greater due to the more complex strain field during bending. Second, recall that in uniaxial tension the strain localizes at $\bar{E}_{Z Z}^{\mathrm{B}}=1 \%$, yet in bending the strain profiles in (2) reach $1.4 \%$ strain at $Y=-C$ prior to any strain localizations. Furthermore, the strain profile near the tube midspan (red line) shows little signs of localization even during (3)-(5). Instead, the outer-fiber tensile strain 
(1)

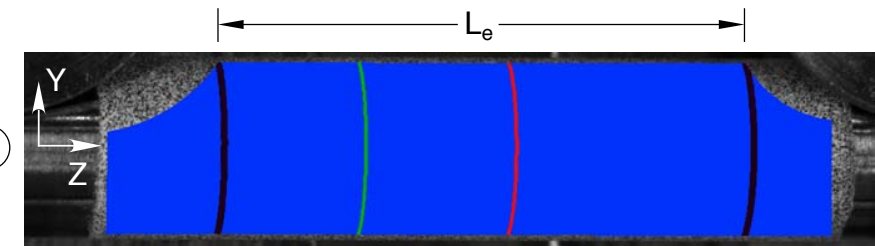

(2)

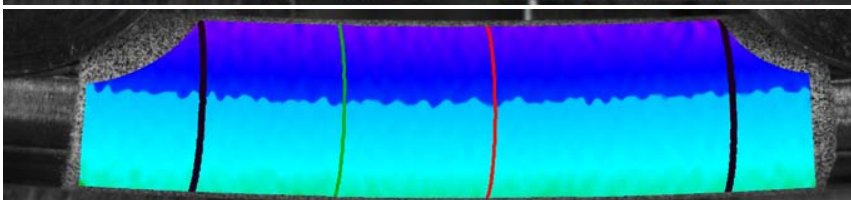

(3)

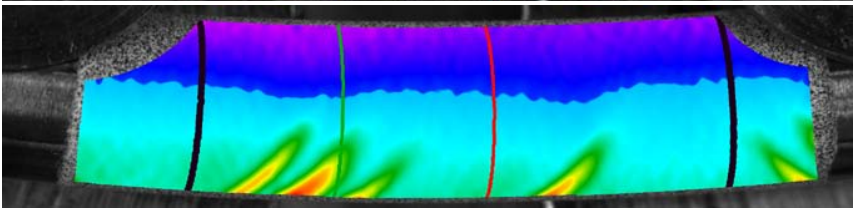

(4)

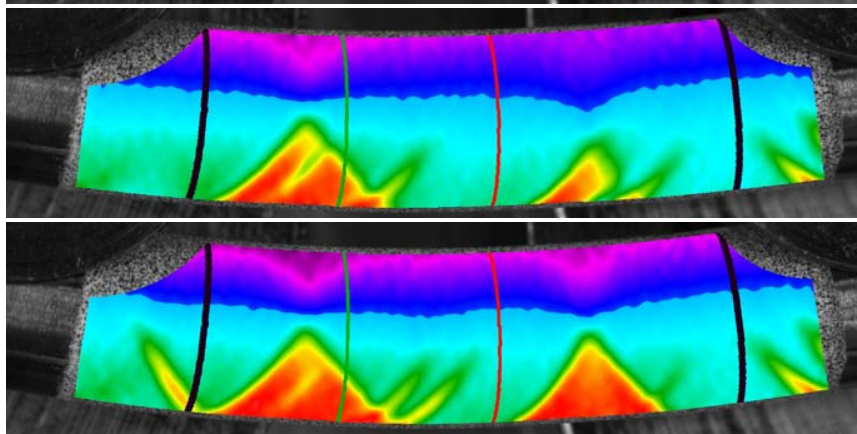

(6)

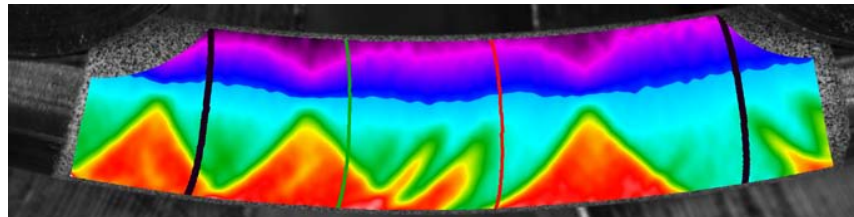

(7)

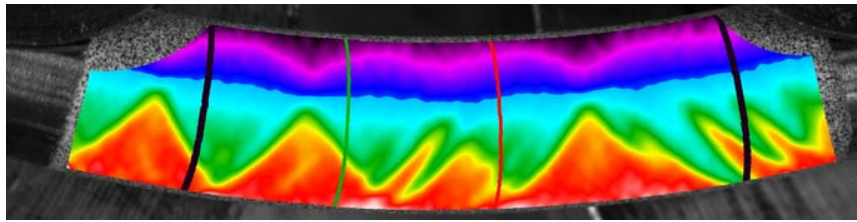

(8)

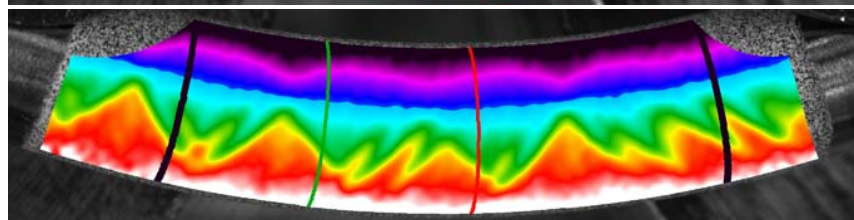

(9)

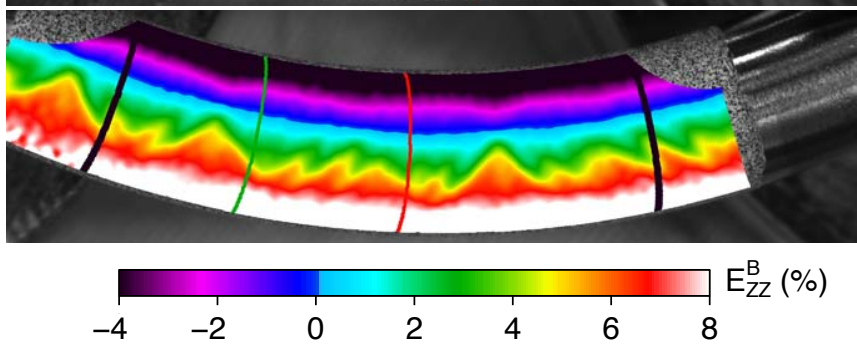

(a)

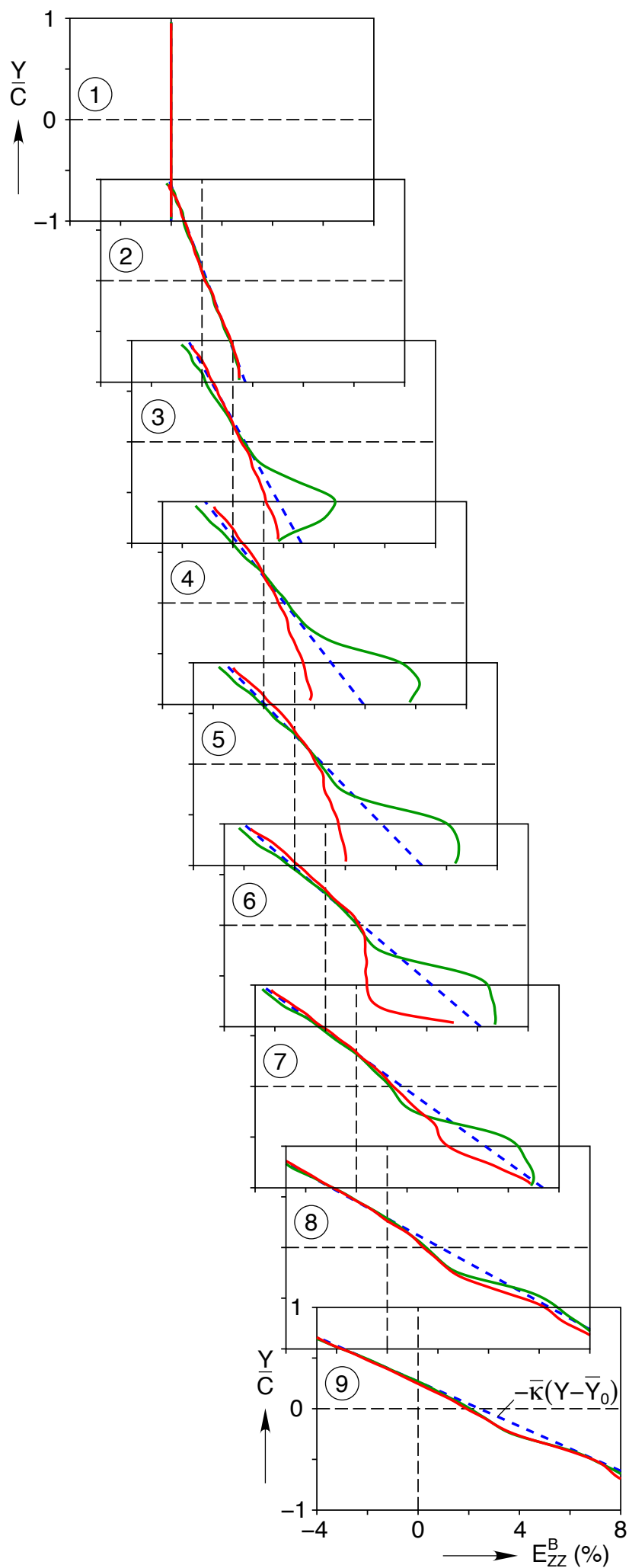

(b)

Figure 11: Experiment B1, loading. (a) Axial strain fields from DIC during loading corresponding to times labeled in Fig. 10, (b) Local axial strain profiles (solid lines) extracted from DIC compared against strain profiles predicted by beam theory (dashed lines), showing that locally plane sections do not remain plane. 

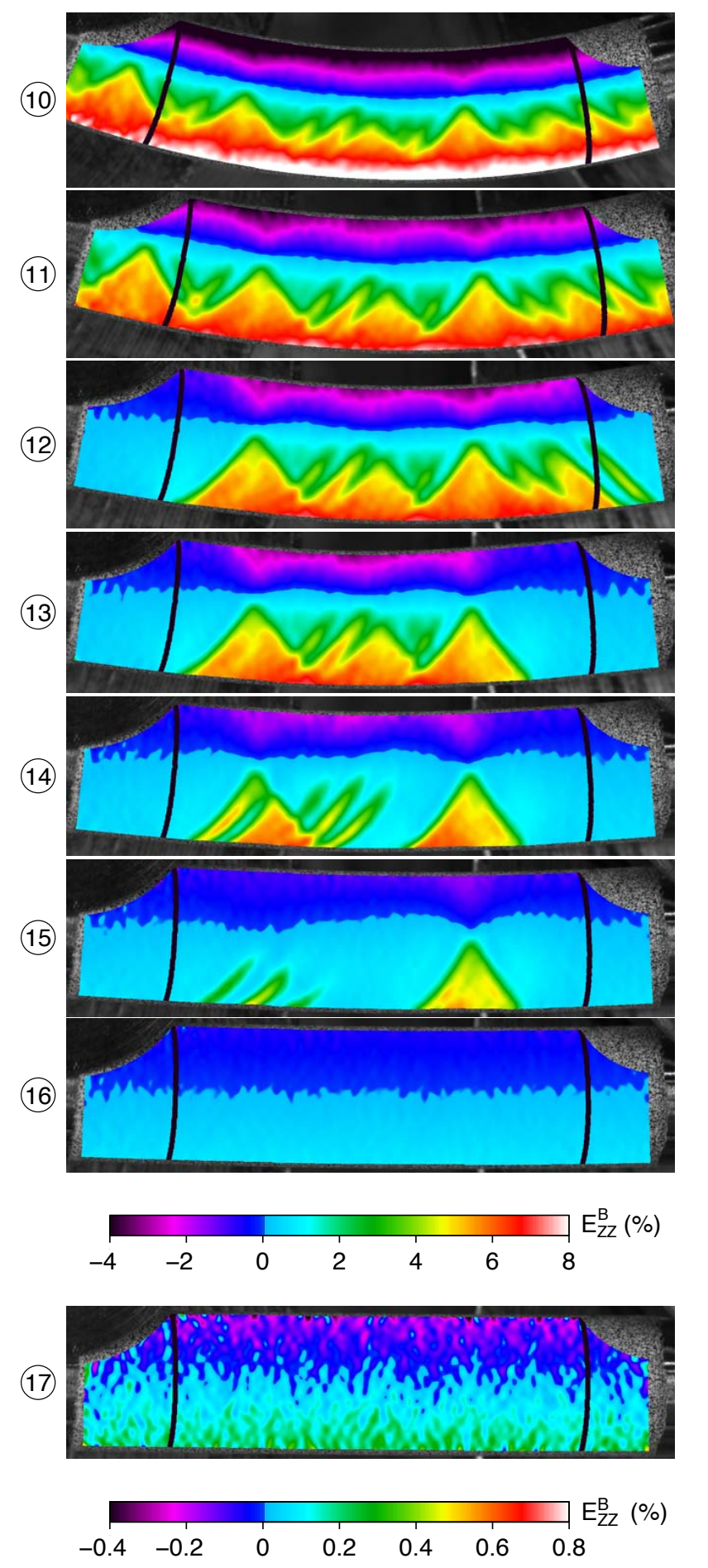

Figure 12: Experiment B1, unloading. Axial strain field images from DIC, corresponding to times labeled in Fig. 10, showing strain localizations disappearing in the reverse order that they were generated during loading. Note the magnified strain scale for the last image (17) after final unloading to zero applied moment. 


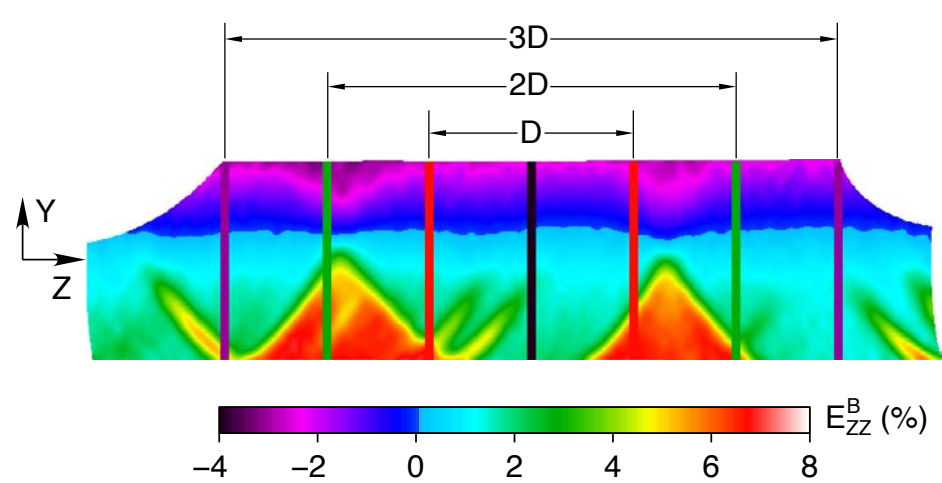

(a)

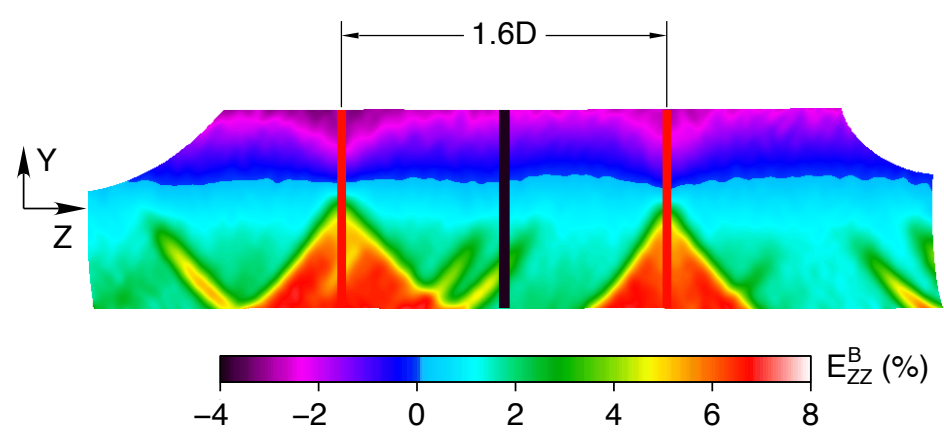

(c)

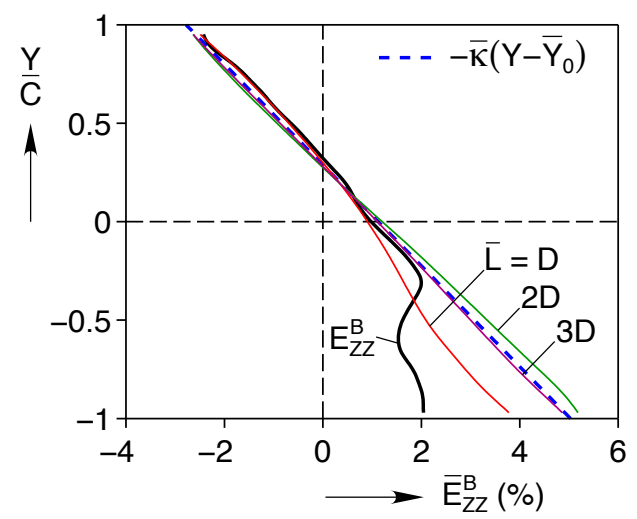

(b)

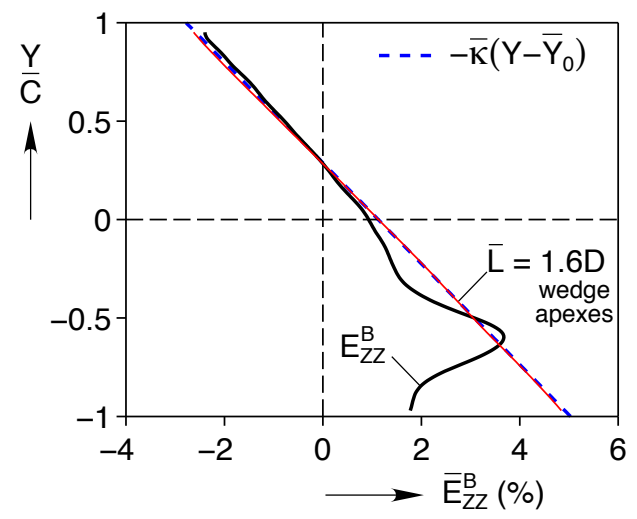

(d)

Figure 13: Experiment B1 at time (5): (a) Same axial strain field as image (5) in Fig. 11a, except shown normal to the bending plane and transformed to the reference frame. (b) Strain profiles at the gage length center $\left(E_{Z Z}^{\mathrm{B}}\right)$ and those averaged across $\bar{L} / D=1,2$, and 3 diameters of gage length corresponding to regions shown in (a). The dashed line is predicted by EulerBernoulli beam theory for comparison. (c) Same axial strain field (5), but showing an averaging region between apexes of wedge localizations. (d) Strain profile along the black line shown in (c) and the averaged strain profile in the region between wedge apexes. This demonstrates that the local strain profile may be very non-linear, but the strain profile matches Euler-Bernoulli beam theory quite well when averaged across a few diameters of axial length.

at $Y=-C$ gets arrested at about $E_{Z Z}^{\mathrm{B}} \approx 2 \%$, while the average curvature of the tube continually increases (see lower end of the red strain profile in Fig. 11(b)). Later, this outer-fiber strain catches up to the strain predicted by beam theory kinematics between (6)-8).

The $2 \%$ strain prior to localization was unexpectedly large, considering that the onset of localization in pure tension occurs at only $1 \%$. The kinematically preferred strain gradient through the diameter of the tube likely suppressed the $\mathrm{A} \rightarrow \mathrm{M}^{+}$instability, pushing the material to $2 \%$ strain until there was sufficient driving force at that location to cause strain localization. One might conclude that the austenite suffered $2 \%$ strain, resulting in a stress at this location of roughly $65.3 \mathrm{GPa} \times 0.02=1.3 \mathrm{GPa}$ (or about $1.05 \mathrm{GPa}$, if accounting for $\approx 0.4 \%$ strain due to the rhombohedral phase). This high tensile stress, however, is not reflected in the moment response between (2) and (7). The discrepancy can be explained if $\mathrm{A} \rightarrow \mathrm{M}^{+}$transformation can occur without localization in tension. It is known that some local pockets of $\mathrm{A} \rightarrow \mathrm{M}^{+}$transformation do occur during tension prior to macroscopic strain localization. Brinson et al. (2004) observed martensite in isolated grains prior to localization using optical microscopy during tensile testing of thin NiTi strips. Accordingly, if isolated grains transformed prior to localization during our bending tests, the local strain could reach $2 \%$ without a huge increase in the local macroscopic stress. This effect could have important implications for bending fatigue, where a material point might be forced to go through this process for millions of cycles in a bending application. Repeatedly suppressing the $\mathrm{A} \rightarrow \mathrm{M}^{+}$instability in bending could cause a very different fatigue performance than what might be predicted from uniaxial data alone.

The strain profiles in Fig. 11(b) clearly show that plane sections do not remain plane locally, however 
the average curvature $\bar{\kappa}$ correctly predicts the average strain gradient across the diameter of the tube. Figure 13(a) shows the axial strain field corresponding to instance (5) in Fig. 10. This is the same strain field as image (5) in Fig. 11(a), except viewed normal to the bending plane and transformed back to the reference frame. Several straight vertical lines are overlaid on the axial strain field. The black line indicates the center of the gage length, while the other pairs of vertical lines indicate three averaging windows, whose widths are $\bar{L}=D, 2 D$, and $3 D$. Figure $13(\mathrm{~b})$ shows the strain profiles corresponding to the black vertical line (local strain profile) and the three averaging windows of Fig. 13(a). Averaged over two diameters of length in the $Z$ direction, the strain profile is nearly linear. Averaged over three diameters the average strain profile overlaps the beam theory prediction, $-\bar{\kappa}\left(Y-\bar{Y}_{0}\right)$, almost perfectly. In fact, a more judicious choice of averaging window is shown in Fig. 13(c) between the apexes of the wedge localizations, which has length of only $\bar{L}=1.6 D$. The corresponding average strain profile is shown in Fig. 13(d), which matches the beam theory profile almost exactly, despite the fact that the local strain profile at the center of this window is very nonlinear since it intersects one of the protruding fingers. Thus, plane sections do remain plane in an average sense, but one should be aware that the average curvature can over/under predict the local strain by as much as two-fold.

Considering the overall evolution of bending strain fields, one might have expected the bending localizations to start at the rollers and propagate inward based on the front propagation in tension (see again Fig. 5). In fact, Kyriakides et al. (2008) measured a flat moment-curvature response at the onset of plasticity in steel tubes with Lüders bands, and observed propagating regions of high curvature. In addition, several researchers (Berg, 1995b; Purohit and Bhattacharya, 2002; Buratti, 2005) have postulated this sort of behavior for bending of SMAs, and have built models to simulate curvature propagation. In our case, the moment-curvature response is always at least slightly positive during loading, suggesting a structurally stable response, and curvature does not appear to propagate here. The stable bending response during loading is likely due to the stable compression response during loading, but it could also be caused by our short free length between the inner rollers, $L / D=4.65$. (Note, the length of tube between the rollers is somewhat longer than it appears in Fig. 13(a), since the cutouts in the strain field are not the actual roller locations. See instead (1) in Fig. 11(a) where the rollers are outside the field of view.) As shown in He and Sun (2010), thin NiTi strips with large aspect ratios (length/width) exhibited front propagation in tension, but specimens with aspect ratios less than 1 had a stable global tensile response and no flat plateaus. It is possible that experiments using longer tube lengths than ours could exhibit propagating curvature.

While we did not observe curvature propagation during loading, there appears to be an axial periodicity to the more developed strain localizations. See, for example, the spacing between the two wedges in image (5) in Fig. 11 and the three wedges in image (2) in Fig. 16b. The spacings are not the same due to the particular locations where localization began, but the size of the wedges do tend to set a characteristic length scale. This distributes the localizations relatively evenly, which is why plane sections can be considered to remain plane in a suitably average sense. Having said that, the bending response during unloading is reasonably flat between (11) and (16). Localizations disappear suddenly during this time, and the strain field sequence in Fig. 12 has some elements of curvature propagation in that strain localization disappears first near the two ends. Consistent with our earlier conjecture that the positive moment-curvature tangent modulus during loading is due to the stable loading response in pure compression, the faint evidence of curvature propagation during unloading may be connected to the flat $\mathrm{M}^{-} \rightarrow \mathrm{A}$ response during unloading in pure compression.

Another phenomenon that was observed during the bending of our tube was the ovalization of the cross-section as shown in Fig. 14(a). This is called the Brazier effect, which is the tendency of tubes, even those made of conventional materials, to ovalize in bending (Brazier, 1927). The cause can be explained with the help of Fig. 14(b), using the co-rotated frame $(x, y, z)$ introduced in Section 3.3. Under large bending deformations, the tensile/compressive normal force resultants on a cross section have a small, yet appreciable, component along the $y$-axis of nearby cross-sections. The force components on the tension (lower) and compression (upper) portions both point towards the neutral axis. Thus, they act to compresses the beam in the $y$-direction, causing thin-walled tube cross-sections to ovalize.

Figure 14(a) displays the ovalized shape of the tube outer diameter at (1), (7), and (9). Note that the shapes are not elliptical, due in part to anti-clastic curvature effects, but the tension-compression asymmetry may also have contributed here. These shapes were generated using the three dimensional DIC displacement data gathered on the $X<0$ side of the tube. The $x$ and $y$ coordinates for each data point were averaged 


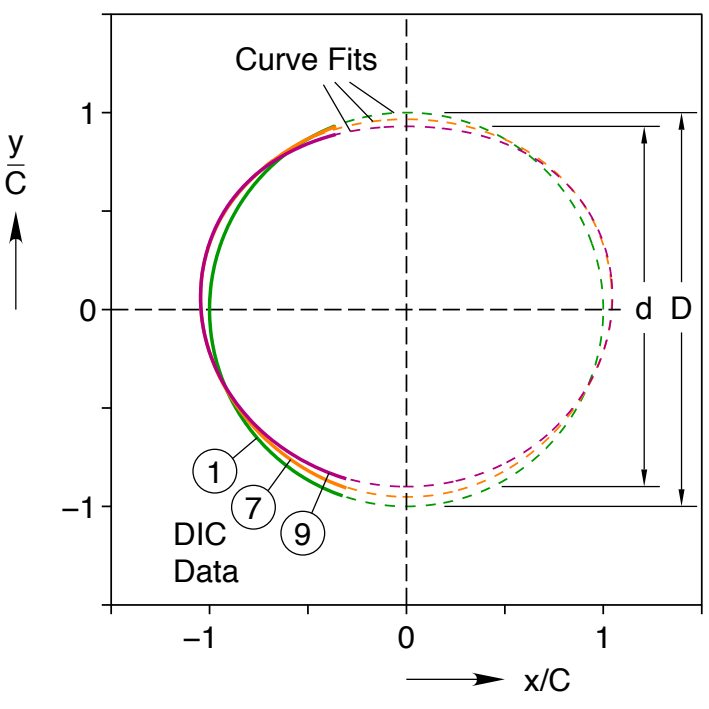

(a)

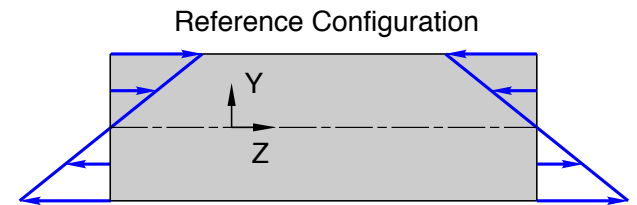

Current Configuration

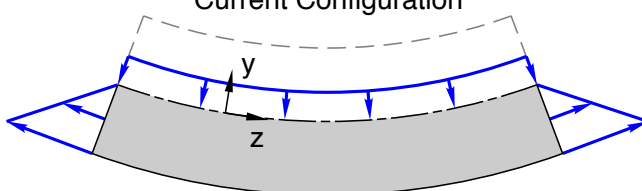

(b)

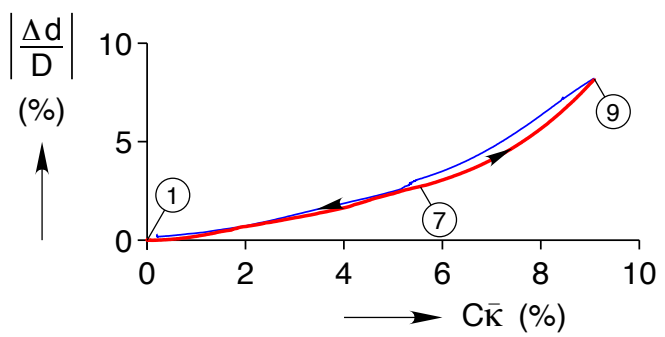

(c)

Figure 14: (a) Experiment B1. Measured (solid lines) ovalized cross-section at three times, (1), (7) and (9) of Fig. 10a, where $C \bar{\kappa}=0 \%, 5.56 \%$, and $9.04 \%$. Dotted lines are extrapolations of the DIC data. (b) Schematic of stresses (linear elastic behavior shown for simplicity) on an undeformed tube element, and on a deformed tube element with an additional cut along the neutral axis. (c) Evolution of percent ovalization versus average bending curvature calculated from the DIC data of Experiment B1.

along the extensometer gage length $L_{\mathrm{e}}$ to generate the solid curves in Fig. 14(a). Then the averaged DIC data was mirrored across the $X=0$ plane and an 8-term Fourier series was fit in polar coordinates to each cross section to generate the dashed curves in Fig. 14(a). This process was repeated for each DIC image during the experiment to find the current vertical deformed diameter $(d)$, which was used to determine the evolution of the percent ovalization of Fig. 14(c). Ovalization progressed right from the start of the experiment, but accelerated near (7) and reached a maximum value of $|\Delta d / D|=8.2 \%$ at (9).

Some small error likely exists in the curves of Fig. 14(a), since it was difficult to pinpoint the exact $x=0$ location of the cross section. The tube moved small amounts, on the order of a few $\mu \mathrm{m}$, in the $X$-direction during bending, and we did not have data around the full tube circumference to distinguish rigid body motion from deformation. We kept this error to a minimum by extrapolating and integrating the hoop strain $\left(E_{\Theta \Theta}^{\mathrm{B}}\right)$ to fill in the missing surface coordinates at the top and bottom of the tube. This gave us a reasonable estimate for the rigid body motion of $x=0$. Also, the uncertainty in the rigid body motion of $x=0$ should have a minimal affect on the percent ovalization, since we defined ovalization as the change in diameter in the $y$-direction.

\subsection{Bending Models}

The question arises whether the uniaxial data presented here can be used to predict the global bending response using simple bending theory, as is often done in engineering practice for SMA bending applications. The answer is yes, provided care is taken. In this sub-section we compare a few candidate models. This exercise also serves as consistency check between our pure-tension, pure-compression, and bending data sets.

Since plane sections remain plane when averaged over a few diameters of tube length, we investigated whether Euler-Bernoulli beam theory can predict the global bending response and the evolution of the average neutral axis based on our uniaxial data. We found that only using the tension data was not sufficient, but an asymmetric model using separate tension and compression data provided a fairly good match with experiment. For simplicity, we only attempted to capture the average moment-curvature response during loading, avoiding for now unloading which would require a path-dependent constitutive model. Three bending models were generated from our uniaxial data: 


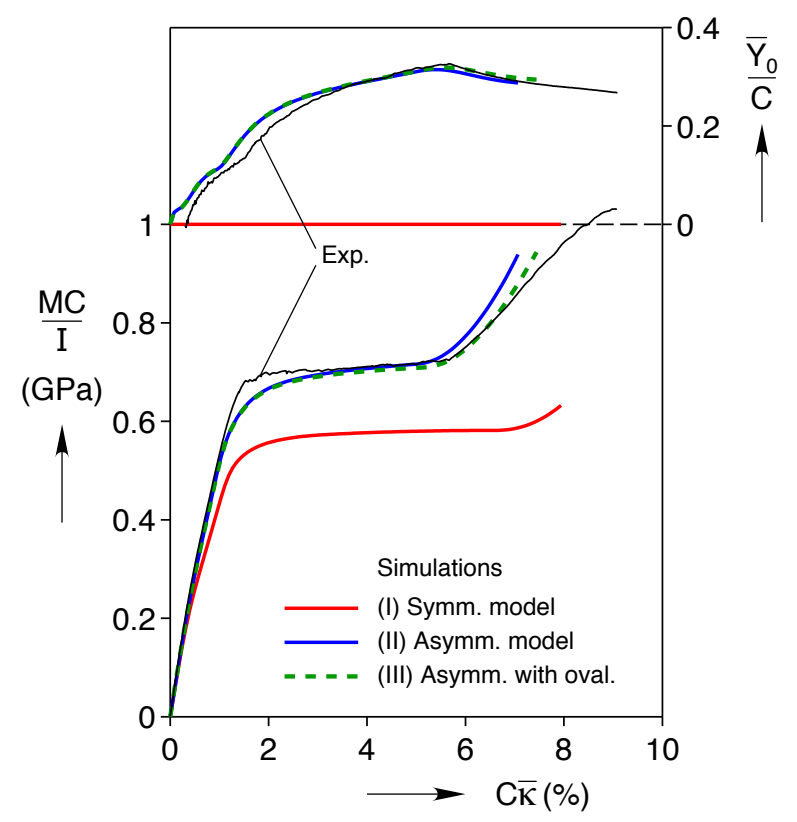

Figure 15: Comparison of measured bending response (Exp.) during loading to simulations using three bending models based on the uniaxial data (T2 and C3) of Fig. 8(a). Model (I) assumes symmetric tension-compression behavior. Model (II) uses asymmetric tension-compression behavior. Pseudo-model (III) uses the asymmetric model but accounts for the measured average ovalization of the tube cross-section (see Fig. 14(a)).

(I) a symmetric model using only the tension data, mirrored for the compression side $\left(\Pi^{-}=-\Pi^{+}\right)$,

(II) an asymmetric model using the separate tension and compression data,

(III) an asymmetric pseudo-model that accounts for the Brazier effect on the bending moment. This last one is termed a "pseudo-model", since ovalization data from the bending experiment were used. It is not really a predictive model, but rather a consistency check on our data.

The assumed Biot strain distribution through the cross section was given in eq. (3). Treating the local bending stress as purely uniaxial,

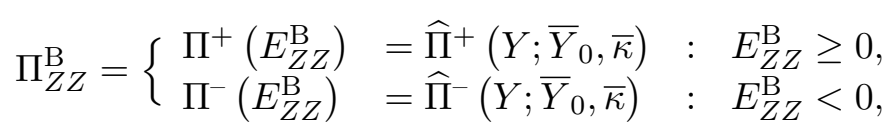

where functions $\Pi^{+}$and $\Pi^{-}$use the respective averaged uniaxial loading responses from the high-strain tension and compression experiments of Section 4. The loading portions of T2 (895 data points) and C2 (255 data points) in Fig. 8 were linearly interpolated for $\Pi^{+}$and $\Pi^{-}$, respectively. The small load drop at the end of the plateau of the solid red curve was ignored, since the drop is due to front coalescence and we neglect strain localization in these models.

Axial equilibrium is simply $P_{z}=0$, and moment equilibrium is just a constant bending moment $M_{x}=M$, where $M$ is the applied (external) moment and $P_{z}$ and $M_{x}$ are the respective force and moment resultants on a given cross-section of constant $Z$. This gives the following two equations that were used to solve for the two unknowns, $\bar{Y}_{0}$ and $M$, at each time in the loading process,

$$
\begin{aligned}
0 & =\int_{A_{0}} \widehat{\Pi}\left(Y ; \bar{Y}_{0}, \bar{\kappa}\right) \mathrm{d} A, \\
M & =-\int_{A_{0}} Y \widehat{\Pi}\left(Y ; \bar{Y}_{0}, \bar{\kappa}\right) \mathrm{d} A .
\end{aligned}
$$

Integration was numerically calculated over the reference cross-sectional area with $\mathrm{d} A_{0}=\mathrm{d} X \mathrm{~d} Y$ by adaptive Simpson quadrature (quad function in Matlab R2010b (2010)).

A comparison of the three models against the experimentally measured bending data (loading only) is provided in Fig. 15, which shows that the symmetric model (I) significantly under-predicts the overall 
moment response. This model predicts (1) a somewhat lower stiffness and a less linear response during the pre-plateau regime deviating from the experiment prior to $C \bar{\kappa}=1.0 \%,(2)$ a normalized plateau moment of $577 \mathrm{MPa}$ during $\mathrm{A} \rightarrow \mathrm{M}^{+}$phase transformation, lower than the actual value of $712 \mathrm{MPa}$ by about $19 \%$, and (3) a delayed termination of the moment plateau at $C \bar{\kappa}=6.7 \%$, compared to the measured value of $5.66 \%$. The only feature reasonably predicted by this model is perhaps the curvature value at the onset of the moment plateau $(C \bar{\kappa}=1.4 \%$ predicted, versus $1.53 \%$ actual $)$. The symmetric model also predicts a neutral axis that remains at the tube centerline $Y_{0}=0$, which of course, is not the case in the experiment.

The asymmetric model (II) produces a good match with the experimental bending response, both in terms of the average moment-curvature response and the evolution of the neutral axis. The initial preplateau response is matched very well up to an average curvature of about $C \bar{\kappa}=1.0 \%$. Thus, the slight R-phase tension-compression asymmetry at $\bar{E}_{Z Z}^{\mathrm{B}}=0.2 \%$ in Fig. $8(\mathrm{a})$ is also confirmed in the bending data. The level of the moment plateau is matched almost exactly. Considering the very non-uniform strain fields in Fig. 11, the level of agreement (at least up to about $C \bar{\kappa}=5.3 \%$ ) is better than expected.

Certain details of the measured response are not captured by model II, such as (1) the sharp onset of the moment plateau for which the model predicts a more gentle "knee" in the curve between $C \bar{\kappa}=1.0 \%$ and $3.1 \%,(2)$ the modest over-prediction of the neutral axis location for average curvatures between $1.0 \%$ and $3.1 \%$, and (3) the somewhat premature termination of the moment plateau at $C \bar{\kappa}=5.3 \%$ (compared to the measured value of $5.66 \%$ ). Regarding discrepancies (1) and (2), recall that the kinematically preferred linear strain gradient in the bending experiment delayed localization somewhat, which is not captured in the model. As a result, larger stresses were achieved on the tension side in the experiment before localization occurred, resulting in a sharper onset of the moment plateau and a delayed evolution of the neutral axis compared to the model predictions. As for why these discrepancies disappear beyond $C \bar{\kappa}=3.1 \%$, we propose two possible reasons. First, the magnitude of the kinematically preferred strain gradient is larger and becomes more congruous with the strain profile preferred by the material. Second, once a localization has nucleated, it requires little additional stress to to grow, so the "overshoot" in the measured moment response becomes progressively reduced as the bending curvature becomes large and the model prediction catches up to the measured response.

Regarding discrepancy (3), the divergence of the moment prediction by model II above the measured one for $C \bar{\kappa}>5.3 \%$ appears to be due to the ovalization of the tube. As previously mentioned, the rate of ovalization gently starts to accelerate around $C \bar{\kappa}=6 \%$, which is reasonably near $C \bar{\kappa}=5.3 \%$. The pseudo-model (III) was created to take account for ovalization, and it validates our hypothesis as shown in Fig. 15. The DIC data (see again Fig. 14) was used to create a function $y=\hat{y}(Y)$ to link the current $y$ position to the reference $Y$ position. Then, equations (5) and (6) were modified as

$$
\begin{aligned}
0 & =\int_{A_{o}} \widehat{\Pi}\left(\hat{y}(Y) ; \bar{y}_{0}, \bar{\kappa}\right) \mathrm{d} A, \\
M & =-\int_{A_{o}} \hat{y}(Y) \widehat{\Pi}\left(\hat{y}(Y) ; \bar{y}_{0}, \bar{\kappa}\right) \mathrm{d} A,
\end{aligned}
$$

to better account for the strain and moment arm at each point in the ovalized cross-section. We recognize that equations (7) and (8) involve both reference and current quantities, which is rather inelegant. This is done because we are using the measured ovalized cross section from experiment B1 in order to predict $M$ and $\bar{Y}_{0}$ from the same experiment, hence the name "pseudo-model". A true structural model would use equilibrium in the hoop direction to predict the ovalized shape of the cross section and use that to calculate $M$ and $\bar{Y}_{0}$. In our case, material properties were not available in the hoop direction, which are likely quite different from those in the longitudinal direction due to the tube texture and would be difficult to obtain accurately. However, our pseudo-model satisfies our primary aim to verify that our tension, compression, and bending data are consistent.

The pseudo-model nicely captures the termination of the moment plateau, as well as the initial stiffness of the post-plateau moment response. It does, however, start to diverge above the measured response when $C \bar{\kappa}>6.6 \%$. The cause is unclear, but the Brazier effect may again play a role as ovalization becomes extreme. The wall of the tube must locally bend and develop hoop stresses for the cross section to assume an ovalized shape. This more complex biaxial stress state could cause the material to soften in the axial direction, resulting in a lower than predicted moment. It should be mentioned, however, that many SMA 
bending applications use beams of solid cross-sections. If indeed the discrepancy is due to the Brazier effect, it is an issue only for hollow cross-section beams and would not be present for bending of solid cross-section beams. Furthermore, one should recognize this bending experiment is a rather extreme test of the material, considering that superelasticity is rarely used beyond strains of $6 \%$ in practice.

\subsection{Incremental 5-cycle Bending Experiment (B2)}

A second bending experiment (B2) was performed to characterize the incremental cyclic behavior. The moment-curvature response is shown in Fig. 16(a) for five successive cycles, each taken to progressively larger maximum curvatures. The five circled numbers correspond to the strain field images in Fig. 16(b) at the maximum curvature for each cycle. For convenience during this longer bending experiment, the average strain rate at the outermost tensile fiber was about $\pm 4 \times 10^{-5} \mathrm{~s}^{-1}$, twice as fast as in experiment B1. The rate $\pm 4 \times 10^{-5} \mathrm{~s}^{-1}$ is still sufficiently slow to be considered a quasi-static, isothermal experiment. The strain rate sensitivity in bending is far less than in uniaxial tension, since in tension the latent heat is concentrated at the front, while in bending the latent heat exchanges are spread out along the length of the tube and only the outermost tensile fiber experiences the maximum global strain rate. This was verified by overlaying the outer envelope of this moment-curvature response (B2) and the response from experiment B1, which showed a negligible difference.

The moment-curvature data in Fig. 16(a) shows several cyclic shakedown features similar to that seen during cyclic loading of NiTi in unaxial tension:

- The stress at the onset of $\mathrm{A} \rightarrow \mathrm{M}^{+}$transformation shifts downward with each cycle, i.e. exhibits (transformation) cyclic softening, with the largest changes seen during the first few cycles.

- Previous maximum strain points during $\mathrm{A} \rightarrow \mathrm{M}^{+}$are "remembered". The $\mathrm{A} \rightarrow \mathrm{M}^{+}$response in a subsequent cycle rises to meet these stress-strain points of the previous cycle. (Although not done here, if reloading commenced before complete $\mathrm{M}^{+} \rightarrow \mathrm{A}$ transformation, i.e., within the unloading plateau, these "turn-around" points would also be remembered and would affect the response of subsequent unload cycles.)

- The unloading plateau stresses during $\mathrm{M}^{+} \rightarrow \mathrm{A}$ transformation decrease slightly with cycles when unloading begins from within the plateau region. The unloading plateau stresses are reduced more dramatically when unloading starts from points beyond the initial loading plateau.

These are all generally related to micro-plasticity that occurs predominantly during each high stress $\mathrm{A} \rightarrow \mathrm{M}^{+}$ transformation cycle, which tends to create residual stress fields that "lock-in" martensite. Previous $\mathrm{A} \rightarrow \mathrm{M}^{+}$ cycles reduce the energy barriers for subsequent $\mathrm{A} \rightarrow \mathrm{M}^{+}$transformations, allowing $\mathrm{A} \rightarrow \mathrm{M}^{+}$transformation to occur at progressively lower stresses (reduced thermodynamic driving force) during loading. Previous $\mathrm{A} \rightarrow \mathrm{M}^{+}$cycles, however, increase energy barriers for reverse $\mathrm{M}^{+} \rightarrow \mathrm{A}$ transformation, thereby requiring progressively lower stresses (larger reverse driving force) than before for the reverse transformation during unloading.

The strong strain gradient in bending causes material points at different $Y$ locations to follow distinctly different stress-strain paths. Material points near the neutral axis, for example, only undergo partial transformations and some points nearest the neutral axis do not transform at all. For example, Atanacković and Achenbach (1989) used a model to show that the maximum stress magnitude in the cross section of an SMA beam during unloading can occur far from the outer fiber, similar to what can occur in reverse loading of a plastically bent bar of a conventional metal. Raniecki et al. (2001), in particular, modeled of the effect of SMA hysteresis in bending. Both studies predicted that the unloading moment "plateau" height is lower if the beam is bent to a greater maximum curvature. We see some evidence of that here, with the most prominent effect seen during cycles 4 and 5 where the outer fiber strains have been driven high/low off-scale in the strain images of Fig. 16(b) (well below $-4 \%$ in compression and above $8 \%$ in tension). Thus, plasticity at the outer fibers likely contributes to a lower unloading moment plateau.

Under an incremental cyclic history, the shakedown response of bending is similar to the interesting staircase-like response found in tension of NiTi wires (see, for example, Fig. 17 of Shaw (1997)). The loading plateau here is not truly flat so the effect is not as pronounced, but the same phenomenon is apparent in 


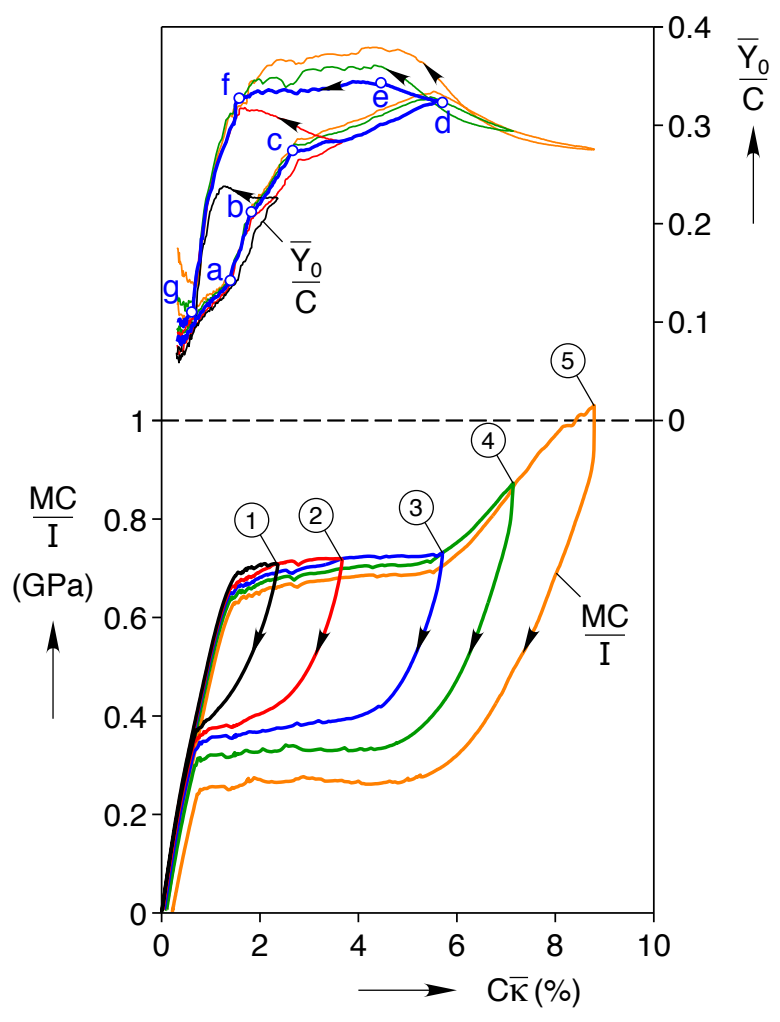

(a)
(1)

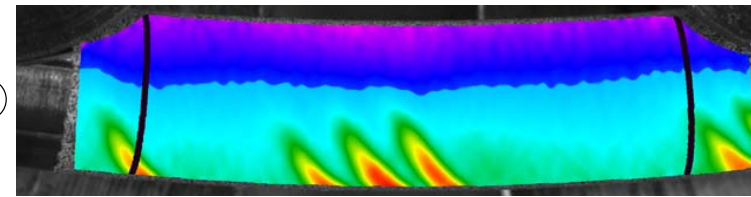

(2)

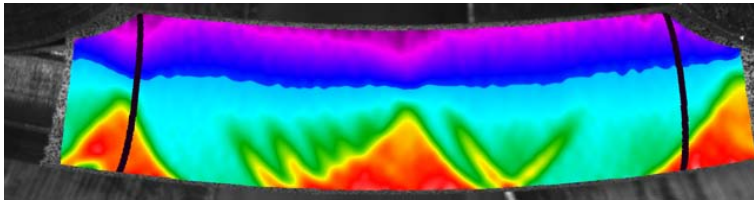

(3)

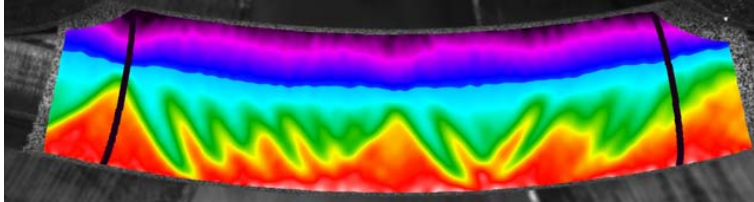

(4)

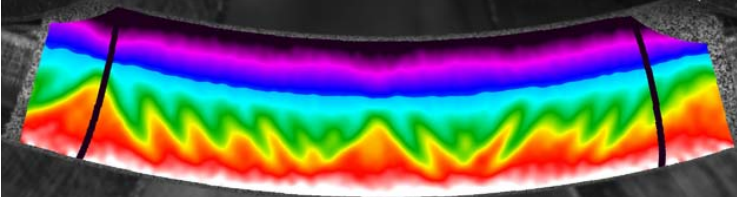

$(5)$

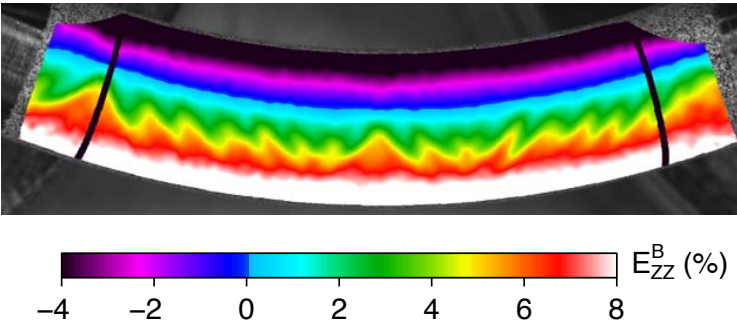

(b)

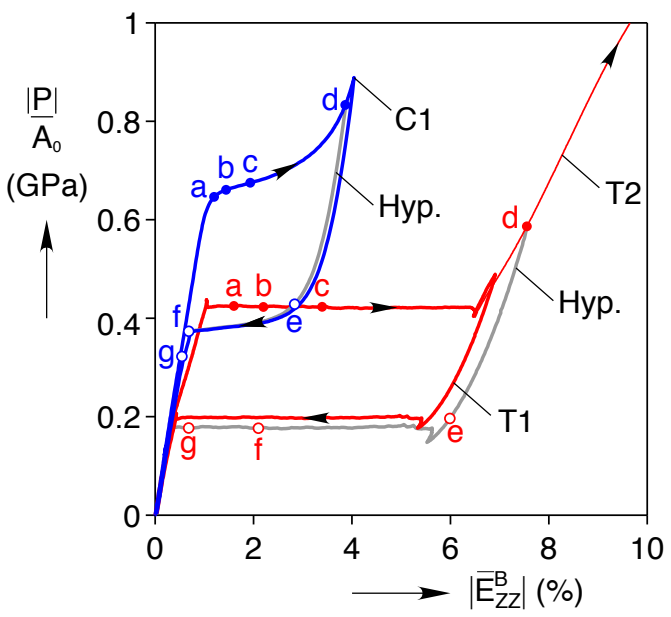

(c)

Figure 16: Experiment B2. (a) Bending moment (lower plot) and neutral axis position (upper plot) versus average curvature for incremental cycling, and (b) corresponding axial strain field images times labeled in (a). Each image was taken at the maximum curvature for that cycle. (c) Predicted outer fiber stress-strain states during cycle 3, showing corresponding points to those of the upper plot of (a). 
Fig. 16(a). As seen in Fig. 16(b), the wedges and fingers of high strain visible in one cycle appear in the same locations during the next cycle (and the sequence is even more obvious in a video of the full set of DIC strain images). The small moment drops associated with localization events also occur at nearly the same values of $C \bar{\kappa}$ each cycle during loading. In other words, if one were to bend a specimen repeatedly to the average curvature of point (2), only the material where the strain localizes in image (2) would experience shake down and the moment at the onset of the plateau would decrease on each cycle. Subsequent bending to larger curvatures would require an increase in moment up to the original value to create localizations in other areas. Accordingly, each labeled point in Fig. 16(a) is a moment-curvature point that is crossed during the next cycle.

The movement of the neutral axis is path dependent at certain stages, and non-monotonic during both loading and unloading. We first describe the data, and then provide explanations for the observations in the next paragraph. Focusing initially on loading only, the upper plots of Figs. 10 and 16(a) both show three stages of neutral axis movement corresponding to the three stages in the moment response. The neutral axis first moves up due to the R-phase non-linearity in tension. Once localization and the moment plateau begins, the neutral axis moves up more rapidly due to the $\mathrm{A} \rightarrow \mathrm{M}$ tension-compression asymmetry. When the moment response stiffens up again (post-plateau), the neutral axis moves back down since the tensioncompression asymmetry is reduced as the tension side reaches high stresses in its post-plateau regime. Next, following the paths of $\bar{Y}_{0}$ during unloading in the upper plot of Fig. 16(a), $\bar{Y}_{0}$ initially continues to move upward in all five cycles. For cycles 3,4 , and $5, \bar{Y}_{0}$ reaches a relatively constant value during much of the moment plateau, but then drops steeply before the end of the moment plateau along a seemingly canonical line common to all unloading cycles. For cycles 1 and 2 , no plateau exists in the $\bar{Y}_{0}$ movement, instead just reaching a local maximum then dropping steeply once it reaches this canonical line.

To explain the evolution of the neutral axis during a load-unload cycle, let us focus on cycle 3 , where the maximum curvature coincided with the end of the loading plateau. Thus this cycle should be relatively free of the significant plasticity effects inherent in the later cycles, which were taken well into the post-plateau regime. The path of the neutral axis during cycle 3 is shown in the upper plot of 16(a) by the bold line and interesting points are labeled $\left(a\right.$ to $g$ ). Using the measured values $\left(\bar{Y}_{0}, \bar{\kappa}\right)$ and eq. (3), the average outer fiber strains at $Y= \pm C$ can be estimated and mapped to stresses on the corresponding uniaxial responses. These points are shown on the uniaxial data in Fig. 16(c). The maximum strains at $d$ do not exactly correspond to the maximum strain for those uniaxial experiments, so estimated (hypothetical) unloading responses are shown by the gray lines. Table 2 provides the estimated values of outer fiber stresses at all these selected points during cycle 3 of Experiment B2, as well as the ratio of magnitudes of compression to tension stresses. While the neutral location depends on the integral of all material points in a cross-section, the extreme values do give a sense of the tension-compression asymmetry and the ratios indeed follow the trends of the neutral axis locations.

- ( $a$ to $c$ ) During loading, the neutral axis moves rapidly upward through points $a, b$, and $c$, which all correspond to $Y=-C$ states along the upper plateau of the tension response. Point $a$, when the neutral axis first accelerates upward, corresponds to the initial knee of the compression side $(Y=C)$ uniaxial response. Point $c$, where the neutral axis slows its upward motion, corresponds to when the compression side reaches its minimum uniaxial tangent modulus.

- ( $d$ to $e$ ) Unloading begins at point $d$, and both the compression and tensile sides have reached stress states in their post-plateau responses. The neutral axis continues to move upward between points $d$ and $e$, during which time $Y= \pm C$ points unload rapidly.

- (e to $f$ ) Point $e$, where the neutral axis stops moving upward, corresponds to the beginning of both compression and tension lower stress plateaus for the outer fiber locations. Between $e$ and $f$ the neutral axis remains relatively static.

- $(f$ to $g)$ Point $f$ is where the neutral axis starts its rapid retreat downward, and this corresponds to the end of lower stress plateau on the compression side. The tension side is still on the lower stress plateau, and does not reach its end until about point $g$. During $f$ to $g$, the tension side stress does not change while the compression side rapidly unloads along its final linear elastic response. The 
Table 2: Estimated stresses at selected points during cycle 3 of Experiment B2

\begin{tabular}{ccccccccc}
\hline & & \multicolumn{7}{c}{ Stresses (MPa) } \\
\cline { 3 - 9 } & & $a$ & $b$ & $c$ & $d$ & $e$ & $f$ & $g$ \\
\cline { 3 - 9 } tension side & $Y=-C$ & 425 & 425 & 425 & 587 & 197 & 177 & 177 \\
compression side & $Y=C$ & -648 & -661 & -675 & -833 & -429 & -374 & -322 \\
\hline asymmetry ratio & $\left|\Pi^{-}\right| / \Pi^{+}$ & 1.52 & 1.56 & 1.59 & 1.42 & 2.18 & 2.11 & 1.82 \\
\hline
\end{tabular}

tension-compression asymmetry is quickly reduced during this time, which explains why the neutral axis moves back downward so rapidly.

Thus, the canonical line seems to exist whenever the tension side is still midway along its lower stress plateau and the compression side has already traversed its lower stress plateau and is unloading along its final unloading segment.

Finally, for cycles 4 and 5 during unloading, it is interesting that the tube unloads to $C \bar{\kappa}=6 \%$ (near point $d$ ) before the hysteresis in $\bar{Y}_{0}$ becomes significant. This is because in the post-plateau regime the change in stress-strain tangent modulus from loading to unloading is roughly the same for tension and compression. It is only when the outer fibers reach the $\mathrm{M} \rightarrow \mathrm{A}$ plateaus in tension and compression that the unloading versus loading behavior is substantially different.

In closing, none of the SMA bending models in the literature capture the localizations shown in Fig. 16(b), which is understandable since the tensile instability makes simulations difficult. However, if one wishes to understand the material response during multiple partial superelastic cycles, tracking strain localizations is important. We note that several studies have shown that even the uniaxial cyclic behavior of SMAs is quite complex. For example, Iadicola and Shaw (2002a) cycled superelastic NiTi wires in tension between maximum and minimum strains within the $\mathrm{A} \rightarrow \mathrm{M}^{+}$plateau, causing the stress to shake down and transformation fronts to sweep back and forth in the same region on the wire. Thus, even for simple tension histories, different material points experience different cyclic histories. Those in the propagating front region experience many transformation cycles, but those outside these regions see perhaps only one transformation cycle. If the elongation subsequently exceeds the previous maximum elongation, and a front must pass through relatively virgin material, the stress jumps back up to the virgin upper plateau level. In fact, during unloading beyond a previous minimum strain (turnaround) point, a significant energy barrier is encountered, current $\mathrm{M}^{+} \rightarrow \mathrm{A}$ fronts are momentarily halted, and the stress drops abruptly and then jumps up again to join the virgin unload plateau as the material nucleates new $\mathrm{M}^{+} \rightarrow \mathrm{A}$ fronts to continue the reverse transformation. Clearly, modeling of SMA cyclic behavior, even in the simplest context (uniaxial tension), is a challenging task. As we have shown, a simple change in the deformation mode (from uniaxial loading to bending) raises a number of new open scientific questions about the precise causes of (perhaps unexpected) phenomena in the structural response. Much work needs to be done to better understand the phenomena in deformation modes other than tension in order to develop modeling tools that can be used with confidence. A definite need exists for more high-fidelity experimental data on the various structural forms of SMA to guide and validate these efforts. The experiments presented herein are a beginning attempt to fulfill that need, and further experiments are planned for the future.

\section{Summary \& Conclusions}

Bending is a fundamental deformation mode of practical importance for superelastic SMAs, but there are few pure bending experiments in the literature, and none that are directly compared to pure tension and compression responses on the same material. We performed isothermal experiments on superelastic NiTi tubes in tension, compression, and large rotation, pure bending using custom-designed fixturing and stereo DIC to quantify the local strain field and eliminate grip effects. The important observations are as follows:

- Localized propagating transformation fronts were observed in uniaxial tension, but not in uniaxial compression. Strain fields in tension showed macroscopic transformation fronts that consisted of crisscrossing fingers of localized strain, largely consistent with previous observations by other researchers, 
but DIC provided quantitative details not measured before. Strain fields in compression were largely uniform, except for some transient buckling during unloading (reverse transformation) which we have not seen reported before.

- The strain fields in bending contained localized strain on the tensile side, but no such localizations on the compression side of the tube. Localization regions started as fine angled fingers that eventually coalesced into wedge regions distributed along the tube length. Due to strain localizations, plane sections clearly did not remain plane locally, causing Euler-Bernoulli beam theory to under/over predict the local strain by as much as $2 \times$. However, if the lateral strain profile is averaged over a few diameters of tube length, then Euler-Bernoulli assumptions are reasonable in an average sense.

- The strain on the tension side reached as high as $2 \%$ in some areas along the tube prior to localizing. This is twice as high as the strain required to nucleate a transformation front in pure uniaxial tension.

- Using the uniaxial tension/compression data as inputs, Euler-Bernoulli beam theory successfully captured the measured moment-average curvature response and neutral axis evolution during loading up to the end of the moment plateau, provided tension-compression asymmetry was included. When the Brazier effect (tube ovalization) was also accounted for, the agreement could be extended somewhat further beyond the moment plateau. Thus, despite the existence of strain localization and the local highly non-linear lateral strain profiles in bending, one can still achieve reasonable global momentcurvature predictions by suitable homogenization of the strain fields along the axial (fiber) direction.

These observations have several implications for further research and device applications.

(1) In many practical situations the moment is not constant along the length of a beam. A small moment gradient could lead to a region of high curvature propagating along the length of the beam as the loading is increased. With localized self heating, three-point bending experiments, for example, could lead to interesting rate effects.

(2) The kinematic constraint of a constant strain gradient in bending is at odds with a localized strain distribution (non-linear strain gradient) created by the material instability. This results in an energetic competition between preferred bending kinematics and localized kinematics, and the material achieves a compromise through a complex morphology of localization (fingers and wedges). A solid cross section beam would strengthen the kinematic constraint and perhaps result in more diffuse localizations or a different morphology from the ones seen here.

(3) Strain localization likely has important implications in cyclic shakedown and fatigue, since local strain levels are relatively uncontrollable by remote boundary conditions. Furthermore, the constrained kinematics of bending delayed localization until tensile strains reached $2 \%$, a full $1 \%$ strain beyond the onset of localization in pure tension. Such suppression of the $\mathrm{A} \rightarrow \mathrm{M}^{+}$instability during bending could lead to very different fatigue performance than that predicted from uniaxial data alone. NiTi is frequently used in implanted medical devices where it must survive millions of superelastic sub-cycles. As such, NiTi fatigue has been heavily studied and several investigators have reported strangely nonmonotonic constant life curves as a function of mean strain (Goodman diagram) in bending (see Adler et al. (2007) for a review). Although most bending-fatigue experiments are done on solid crosssection specimens, which could exhibit a different localization morphology than the ones in our tubes, we suspect the non-monotonic constant life curves are connected to the localizations found here in bending.

Finally, we would like to emphasize that transformational strain localization, now well known to occur in tension of NiTi, can also occur in bending. Strain localization may be ignored to predict the global mechanical behavior for many situations, but this should be done with caution. Localization plays an important role in the details of the strain field and the cyclic histories of various material points may be quite different. This will likely have a strong influence on shakedown evolution and eventual fatigue. 


\section{Acknowledgements}

We gratefully acknowledge the financial support for this work, provided by General Motors Company (through the GM/UM Collaborative Research Laboratory in Smart Materials and Structures), the National Science Foundation (grant CMMI-0727331), and the US Department of Energy, Office of Basic Energy Sciences (contract No. DE-SC0003996 monitored by Dr. John Vetrano). Our thanks go Prof. Kenneth Gall (Georgia Institute of Technology), Jeff Tyber (Tyber Medical LLC), and Hans Jürgen Maier (University of Hannover) for their assistance with obtaining the X-ray texture map of Fig. 8(b) on our NiTi tube specimens. We also appreciate the responsive help by Dr. Hubert Scheirer and other personnel at Correlated Solutions, Inc., who incorporated the Biot Strain into Vic-3D at our request. 


\section{Appendix A. Supplemental Data}

Raw data from all six experiments is hosted at http://dx.doi.org/10.1016/j.jmps.2012.12.012 in comma separated variable (.csv) format. Table A.3 lists the data file name for each experiment, the variables contained in the data file, and the figure(s) the data corresponds to.

Table A.3: Data files for tension (T), compression (C), and bending (B) experiments

\begin{tabular}{lll}
\hline Data File Name & Variables & Corresponding Figure(s) \\
\hline Experiment_T1.csv & & Fig. 4(a), 8(a) \\
Experiment_T2.csv & $t, P / A_{0}, \delta / L, \bar{E}_{\Theta \Theta}^{\mathrm{B}}, \bar{E}_{Z Z}^{\mathrm{B}}, T_{\mathrm{s}}, T_{\mathrm{a}}$ & $\begin{array}{l}\text { Fig. 8(a) } \\
\text { Fig. 4(b), 8(a) } \\
\text { Experiment_C1.csv }\end{array}$ \\
Experiment_C2.csv & Fig. 8(a) \\
\hline Experiment_B1.csv & $t, M C / I, C \bar{\kappa}, \bar{Y}_{0} / C, T_{\mathrm{a}}$ & Fig. 10 \\
Experiment_B2.csv & & Fig. 16(a) \\
\hline
\end{tabular}

Videos of the DIC data for selected experiments are also hosted at the same location. In each video, the mechanical response is synchronized with the $E_{Z Z}^{\mathrm{B}}$ fields throughout the experiment. Table A.4 lists the video file names and the figures the videos correspond to.

Table A.4: Video files for selected tension (T), compression (C), and bending (B) experiments

\begin{tabular}{ll}
\hline Video File Name & Corresponding Figures \\
\hline Experiment_T1.mp4 & Fig. 4(a), 5 \\
Experiment_C1.mp4 & Fig. 4(b), 7 \\
Experiment_B1.mp4 & Fig. 10, 11(a), 12 \\
\hline
\end{tabular}

\section{Appendix B. Comments on the Biot stress \& strain measures}

The Biot strain $\left(\boldsymbol{E}^{\mathrm{B}}\right)$ and stress $\left(\boldsymbol{\Pi}^{\mathrm{B}}\right)$ measures are rarely used for solving boundary value problems in finite deformation mechanics, due to the computational complexity arising from the need to decompose $\boldsymbol{F}$ into $\boldsymbol{R}$ and $\boldsymbol{U}$ (although proponents do exist). Accordingly, these measures may be somewhat unfamiliar, so the aim of this appendix is to justify our choice and to clarify (if only for the humble experimentalist, if not the grizzled theoretician) some implications of their use compared to other measures commonly used. In experiments, one can choose whatever self-consistent stress/strain measures he/she prefers, since the current configuration is not the solution being sought (like when solving a boundary value problem), but is the one actually being measured. We have found that the Biot strain and stress are convenient in certain structural mechanics applications where the loading is co-rotational, such as in this paper, and thus the applied traction vector on a surface element of interest follows the material rotation during the loading process. For example, Irschik and Gerstmayr (2009) nicely showed that for finite deformation of a EulerBernoulli beam, the Biot strain leads to a linear strain distribution through the cross-section; whereas, the more commonly used Green-Lagrange strain leads to a quadratic strain distribution. Thus, the Biot strain is a more natural, and convenient, choice for the bending of slender beams.

Recalling eq. (1), the Biot strain tensor definition is

$$
\boldsymbol{E}^{\mathrm{B}} \equiv \boldsymbol{U}-\boldsymbol{I}
$$

The conjugate Biot stress tensor $\left(\boldsymbol{\Pi}^{\mathrm{B}}\right)$ is defined such that the mechanical power per unit reference volume $\left(\hat{\mathcal{P}}_{0}\right)$ is

$$
\hat{\mathcal{P}}_{0}=\boldsymbol{\Pi}^{\mathrm{B}}: \frac{\mathrm{d} \boldsymbol{E}^{\mathrm{B}}}{\mathrm{d} t}=\boldsymbol{\Pi}^{\mathrm{B}}: \dot{\boldsymbol{U}} .
$$




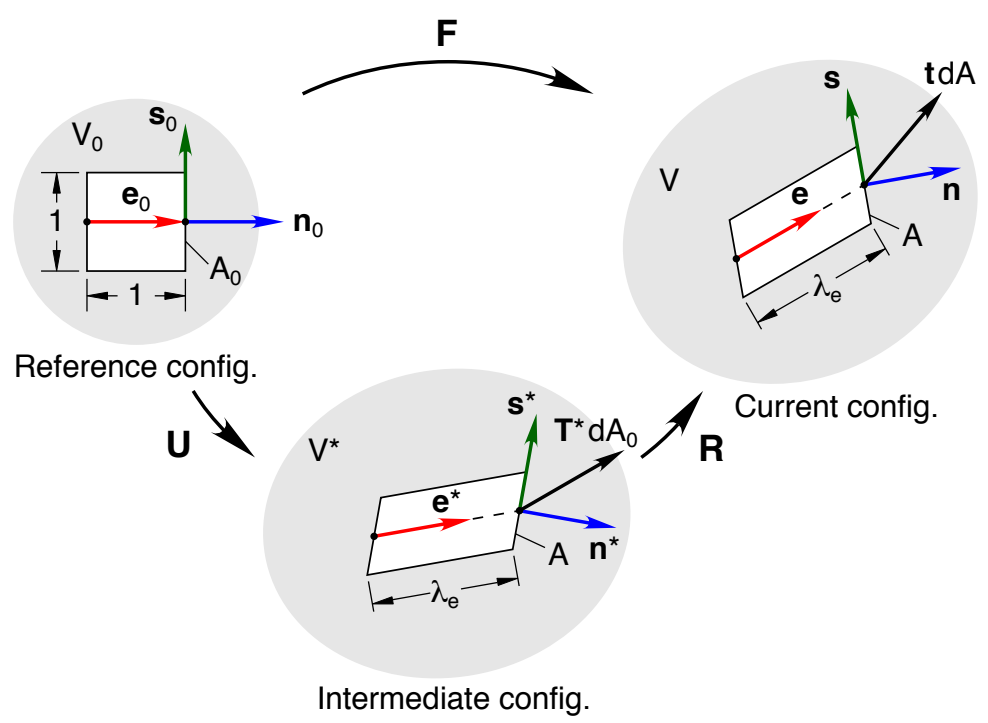

Figure B.17: Schematic of finite deformation, showing the various convected unit vectors $\boldsymbol{e}$ (line element), $\boldsymbol{n}$, (surface normal) and $s$ (surface tangent).

Obviously due to the symmetry of $\boldsymbol{U}$, only the symmetric part of the Biot stress contributes to the mechanical power, so it is common to define a symmetrized Biot stress (or Jaumann stress) as

$$
\boldsymbol{\Pi}_{\mathrm{sym}}^{\mathrm{B}}=\frac{1}{2}\left(\boldsymbol{N} \cdot \boldsymbol{R}+\boldsymbol{R}^{T} \cdot \boldsymbol{N}^{T}\right),
$$

where $\boldsymbol{N}$ is the nominal stress tensor (related to the 1st Piola-Kirchhoff stress tensor by $\boldsymbol{N}=\boldsymbol{P}^{T}$ ). We prefer, however, to use the unsymmetrized Biot stress tensor definition ${ }^{7}$ as

$$
\boldsymbol{\Pi}^{\mathrm{B}}=\boldsymbol{N} \cdot \boldsymbol{R},
$$

which has a more physical interpretation as shown below. The nominal stress tensor is related to the Cauchy stress tensor $(\boldsymbol{\sigma})$ by $\boldsymbol{N}=J \boldsymbol{F}^{-1} \cdot \boldsymbol{\sigma}$ with $J \equiv \operatorname{det} \boldsymbol{F}$. It is well known that eq. (B.2) is equivalent to the alternate forms $\hat{\mathcal{P}}_{0}=J \boldsymbol{\sigma}: \boldsymbol{D}=\boldsymbol{N} \cdots \dot{\boldsymbol{F}}=\boldsymbol{S}: \dot{\boldsymbol{E}}^{\mathrm{L}}$, where $\boldsymbol{D}$ is the rate of deformation tensor, $\boldsymbol{S}$ is the $2^{\text {nd }}$ Piola-Kirchhoff stress tensor, and $\boldsymbol{E}^{\mathrm{L}}$ is the Green-Lagrange strain tensor ${ }^{8}$.

Continuum mechanics is typically concerned with reference (undeformed) and current (deformed) configurations, but it is sometimes convenient to consider the intermediate (rotationless, pure deformation) configuration (see configuration $V^{*}$ in Fig. B.17). By the polar decomposition theorem $\boldsymbol{F}=\boldsymbol{R} \cdot \boldsymbol{U}$, a general deformation $\boldsymbol{F}$ is uniquely decomposed into $\boldsymbol{U}$, which stretches the body into the intermediate configuration, and $\boldsymbol{R}$, which subsequently rotates the body into the current configuration. In this manner, a surface normal can be thought as being transformed from $\boldsymbol{n}_{\mathbf{0}}$ in the reference configuration $\left(V_{0}\right)$, to $\boldsymbol{n}^{*}$ in the stretched configuration $\left(V^{*}\right)$, and then to $\boldsymbol{n}=\boldsymbol{R} \cdot \boldsymbol{n}^{*}$ in the current configuration $(V)$. Forces, on the other hand, reside only in the current configuration, but in order to work with the other configurations one creates fictitious forces (often back-convected somehow). For a given material surface element $\mathrm{d} A$ in the current configuration, loaded by a contact force $\mathrm{d} \boldsymbol{f}$, the current (Eulerian) traction vector is $\boldsymbol{t} \equiv \mathrm{d} \boldsymbol{f} / \mathrm{d} A$. As is commonly done, a pseudotraction per unit reference area $\left(\boldsymbol{t}_{0}\right)$ can be defined by $\boldsymbol{t} \mathrm{d} A=\boldsymbol{t}_{0} \mathrm{~d} A_{0}$, which preserves both the magnitude and direction of the actual force. In certain circumstances it may be convenient (and possibly realistic for a particular time-dependent loading) to consider the co-rotated force vector $\left(\mathrm{d} \boldsymbol{f}^{*}\right)$, such that the force vector element is convected (by rotation only) from this intermediate configuration to the current configuration by $\mathrm{d} \boldsymbol{f}=\boldsymbol{R} \cdot \mathrm{d} \boldsymbol{f}^{*}$, as was done for the surface normal $\boldsymbol{n}^{*}$. One then defines an

\footnotetext{
${ }^{7}$ Note Irschik and Gerstmayr (2009) define an alternate Biot stress tensor, which is the transpose of ours.

${ }^{8}$ The operation $\boldsymbol{C}=\boldsymbol{A} \cdot \boldsymbol{B}$ between two 2nd order tensors $(\boldsymbol{A}$ and $\boldsymbol{B})$ is the contraction resulting in a 2nd-order tensor with components $C_{i j}=A_{i k} B_{k j}$ with respect to a fixed cartesian frame. Double contraction operations result in scalar quantities and are denoted $\boldsymbol{A} \cdot \boldsymbol{B}=\operatorname{tr}(\boldsymbol{A} \cdot \boldsymbol{B})=A_{i k} B_{k i}$ and $\boldsymbol{A}: \boldsymbol{B}=\operatorname{tr}\left(\boldsymbol{A} \cdot \boldsymbol{B}^{T}\right)=A_{i k} B_{i k}$.
} 


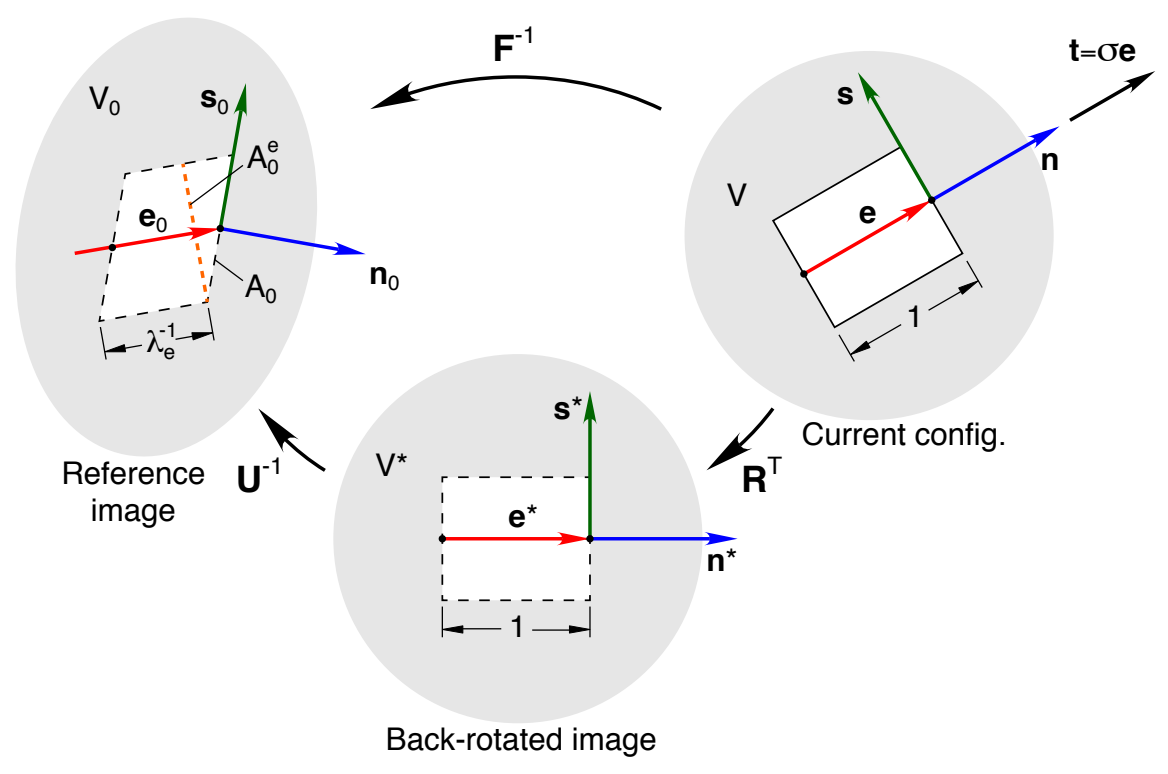

Figure B.18: Schematic of uniaxial Cauchy stress applied to an anisotropic material and its back-rotated and reference images.

associated co-rotated (referential) pseudotraction vector $\left(\boldsymbol{T}^{*}\right)$ such that

$$
\mathrm{d} \boldsymbol{f}^{*}=\boldsymbol{T}^{*} \mathrm{~d} A_{0} .
$$

This gives the following relations between the different tractions,

$$
\mathrm{d} \boldsymbol{f}=\boldsymbol{t} \mathrm{d} A=\boldsymbol{t}_{0} \mathrm{~d} A_{0}=\boldsymbol{R} \cdot \boldsymbol{T}^{*} \mathrm{~d} A_{0} .
$$

One should recognize that $\boldsymbol{T}^{*}$ is distinct from the pseudotraction vector $\left(\boldsymbol{T}_{0} \equiv \mathrm{d} \hat{\boldsymbol{f}}_{0} / \mathrm{d} A_{0}\right)$ commonly used to define the $2^{\text {nd }}$ Piola-Kirchhoff stress, where $\mathrm{d} \boldsymbol{f}=\boldsymbol{F} \cdot \mathrm{d} \hat{\boldsymbol{f}}_{0}$ and $\boldsymbol{T}_{0}=\boldsymbol{n}_{0} \cdot \boldsymbol{S}$, which does not preserve the magnitude of the force. Substituting Cauchy's formula into eq. (B.6) gives the relations

$$
\boldsymbol{n} \cdot \boldsymbol{\sigma} \mathrm{d} A=\boldsymbol{n}_{0} \cdot \boldsymbol{N} \mathrm{d} A_{0}=\boldsymbol{R} \cdot \boldsymbol{T}^{*} \mathrm{~d} A_{0} .
$$

Premultiplying the second relation by $\boldsymbol{R}^{T}$ and rearranging, gives the result

$$
\boldsymbol{T}^{*}=\boldsymbol{n}_{0} \cdot(\boldsymbol{N} \cdot \boldsymbol{R})=\boldsymbol{n}_{0} \cdot \boldsymbol{\Pi}^{\mathrm{B}} .
$$

Thus, the quantity $\Pi^{\mathrm{B}} \equiv \boldsymbol{N} \cdot \boldsymbol{R}$ plays the same role as $\boldsymbol{N}$ and $\boldsymbol{\sigma}$ in a Cauchy relation to its respective traction vector. Referring to the deformation schematic in Fig. B.17, the normal and shear components of $\boldsymbol{T}^{*}=\sigma_{0}^{*} \boldsymbol{n}^{*}+\tau_{0}^{*} s^{*}$ can be calculated as

$$
\begin{aligned}
& \sigma_{0}^{*}=\boldsymbol{n}_{0} \cdot \boldsymbol{\Pi}^{\mathrm{B}} \cdot \boldsymbol{n}^{*}, \\
& \tau_{0}^{*}=\boldsymbol{n}_{0} \cdot \boldsymbol{\Pi}^{\mathrm{B}} \cdot \boldsymbol{s}^{*} .
\end{aligned}
$$

It should be noted that a convected line element $\boldsymbol{U} \cdot \boldsymbol{e}_{0}=\lambda_{e} \boldsymbol{e}^{*}$, where $\lambda_{e}$ is the stretch ratio of that line element, may not be co-linear with the normal $\boldsymbol{n}^{*}$.

If, however, $\boldsymbol{n}^{*}$ is a principal axis of $\boldsymbol{U}$, now thinking of elementary beam theory where shear deformation is neglected, then the axis remains co-linear with the surface normal, $\boldsymbol{e}^{*}=\boldsymbol{n}^{*}=\boldsymbol{n}_{0}$, and in an orthogonal direction $s^{*}=s_{0}$. So in this special case, we have

$$
\begin{aligned}
\sigma_{0}^{*} & =\boldsymbol{n}_{0} \cdot \boldsymbol{\Pi}^{\mathrm{B}} \cdot \boldsymbol{n}_{0}, \\
\tau_{0}^{*} & =\boldsymbol{n}_{0} \cdot \boldsymbol{\Pi}^{\mathrm{B}} \cdot \boldsymbol{s}_{0} .
\end{aligned}
$$

Besides bending, other situations may exist where the Biot stress/strain measures are useful, such as uniaxial loading of an anisotropic material as considered in Appendix D. In this case, the traction vector 
on surface element with current normal $\boldsymbol{e}$ is $\boldsymbol{t}=\sigma \boldsymbol{e}$ and the uniaxial Cauchy stress tensor is $\boldsymbol{\sigma}=\sigma \boldsymbol{e} \otimes \boldsymbol{e}$ (see Fig. B.18). (This, of course, presumes that a suitable loading device can be constructed to achieve such a simple stress state - in practice, not a trivial task for experiments on anisotropic materials.) Thinking of our tension experiments on NiTi where transformation occurs at constant tension force, the current Cauchy stress $(\sigma)$ is not constant during deformation. Instead, we seek to hold the force $(\sigma \mathrm{d} A)$ constant, which is more naturally done with one of the referential stress measures. Nanson's formula relates the oriented current area to the back-convected reference area as

$$
\boldsymbol{n} \mathrm{d} A=J \boldsymbol{F}^{-T} \cdot \boldsymbol{n}_{0} \mathrm{~d} A_{0} .
$$

The reference pseudo-traction vector is $\boldsymbol{t}_{0}=\sigma_{0} \boldsymbol{e}$ such that eq. (B.6) is satisfied to give the correct force. Taking $\boldsymbol{e}=\boldsymbol{n}$ and using eqs. (B.6) and (B.13) give

$$
\sigma_{0}=J \sigma \lambda_{e}^{-1} \boldsymbol{e}_{0} \cdot \boldsymbol{n}_{0}
$$

where $\boldsymbol{F}^{-1} \cdot \boldsymbol{e}=\lambda_{e}^{-1} \boldsymbol{e}_{0}$ is the back-convected reference line element and $\lambda_{e}$ is its stretch ratio. Again, it should be emphasized that in general $\boldsymbol{e}_{0} \neq \boldsymbol{n}_{0}$, i.e. $\boldsymbol{n}_{0}$ is not simply the back-convected unit vector of material line element, but instead depends on the cross product of two orthogonal vectors that define a surface element. This makes the interpretation of $\sigma_{0}$ problematic, since it is the current normal force in direction $\boldsymbol{n}$ divided by a possibly tilted reference area image $A_{0}$ associated with normal $\boldsymbol{n}_{0}$. The relationship between $\boldsymbol{n}$ and $\boldsymbol{n}_{0}$ can be found through eq. (B.13) after dotting both sides with $\boldsymbol{n}$ to find the the relation between $\mathrm{d} A$ and $\mathrm{d} A_{0}$, but the result is somewhat messy. The line elements along $\boldsymbol{e}$ and $\boldsymbol{e}_{0}$ have the simpler relationship $\lambda_{e} \boldsymbol{e}=\boldsymbol{F} \cdot \boldsymbol{e}_{0}$, so the uniaxial reference stress measures can be easily defined in terms of these unit vectors instead. Note that reference area element $\mathrm{d} A_{0}$ with unit normal $\boldsymbol{n}_{0}$ is related to the projected reference area element $\mathrm{d} A_{0}^{e}$ having unit normal $\boldsymbol{e}_{0}$ (see again Fig. B.18) by

$$
\mathrm{d} A_{0}^{e}=\boldsymbol{e}_{0} \cdot \boldsymbol{n}_{0} \mathrm{~d} A_{0} .
$$

Using eqs. (B.14) and (B.15), the force magnitude becomes $\sigma_{0} \mathrm{~d} A_{0}=J \sigma \lambda_{e}^{-1} \mathrm{~d} A_{0}^{e}$, so it is convenient to define a new reference stress as $\sigma_{0}^{e} \equiv J \sigma \lambda_{e}^{-1}$ that is associated with area element $\mathrm{d} A_{0}^{e}$. In this way the force magnitude is still preserved, $\sigma_{0} \mathrm{~d} A_{0}=\sigma_{0}^{e} \mathrm{~d} A_{0}^{e}$. The nominal and Biot stress tensors then take the simple forms

$$
\begin{aligned}
\boldsymbol{N} & =\sigma_{0}^{e} \boldsymbol{e}_{0} \otimes \boldsymbol{e}, \\
\boldsymbol{\Pi}^{\mathrm{B}} & =\sigma_{0}^{e} \boldsymbol{e}_{0} \otimes \boldsymbol{e}^{*},
\end{aligned}
$$

where $\boldsymbol{e}^{*}=\boldsymbol{R}^{T} \cdot \boldsymbol{e}$ is the unit vector of a back-rotated line element to the intermediate configuration $\left(\mathrm{V}^{*}\right)$. This shows that both the nominal stress and Biot stress tensors are clearly two-point tensors, where the nominal stress has footing in configurations $V_{0}$ and $V$, while the Biot stress has footing in configurations $V_{0}$ and $V^{*}$. Certainly, the components of both tensors defy a simple physical interpretation in the most general case, and neither tensor is symmetric. While the Biot stress may be weirdly dependent on $\boldsymbol{U}$, its definition at least removes the dependence of its components on the rotation $\boldsymbol{R}$.

If, however, we consider uniaxial loading with force resultant $P$ in the $Z$-direction and the material is at least orthotropic about this direction, then $\boldsymbol{e}_{Z}$ is a principal direction of $\boldsymbol{U}, \boldsymbol{R}=\boldsymbol{I}$, and $\boldsymbol{n}_{0}=\boldsymbol{e}_{Z}$ remains fixed during loading. The only non-zero stress component is $\sigma_{0}=\Pi_{Z Z}^{\mathrm{B}}=N_{Z Z}$, and when averaged over the cross-section, is the just engineering stress $P / A_{0}$.

\section{Appendix C. A simple model based on a Gibb's mixure rule}

A first-order, uniaxial superelastic model can be constructed from a simple Gibbs mixture rule,

$$
g(\sigma, T, \xi)=(1-\xi) g_{A}(\sigma, T)+\xi g_{M}(\sigma, T)
$$


where $g$ is the total specific (per unit mass) Gibbs free energy, $\sigma$ is the uniaxial stress, $T$ is the thermodynamic temperature, $\xi$ is the mass fraction of martensite, and $g_{A}, g_{M}$ are the specific Gibbs free energies of the two pure phases austenite and martensite. For the pure phases, the following forms are chosen,

$$
\begin{aligned}
-g_{A} & =\frac{\sigma^{2}}{2 \rho E}+s_{0}\left(T-T_{0}\right)+c_{0}\left[T \ln \left(\frac{T}{T_{0}}\right)-\left(T-T_{0}\right)\right], \\
-g_{M} & =\beta \frac{\sigma}{\rho}+\frac{\sigma^{2}}{2 \rho E}+\left(s_{0}+\Delta s\right)\left(T-T_{0}\right)+c_{0}\left[T \ln \left(\frac{T}{T_{0}}\right)-\left(T-T_{0}\right)\right],
\end{aligned}
$$

with material constants: elastic modulus $(E)$, mass density $(\rho)$, stress-free equilibrium transformation temperature $\left(T_{0}\right)$, specific heat $\left(c_{0}\right), \mathrm{A} \rightarrow \mathrm{M}$ transformation strain $(\beta)$, and $\mathrm{A} \rightarrow \mathrm{M}$ entropy difference $(\Delta s<0)$. This model neglects thermal expansion, some differences in A and $\mathrm{M}$ properties (i.e., elastic modulus, density, and specific heat), and any interfacial energy terms, all of which we accept as a first approximation. The specific driving force for $\mathrm{A} \rightarrow \mathrm{M}$ transformation $(\mu \equiv-\partial g / \partial \xi)$ is calculated as

$$
\mu=g_{A}-g_{M}=\beta \frac{\sigma}{\rho}-\frac{\Lambda}{T_{0}}\left(T-T_{0}\right),
$$

where the specific entropy jump $-\Delta s \approx \Lambda / T_{0}$ has been substituted in terms of the latent heat of transformation $(\Lambda>0$, a material constant). Assuming $\mathrm{A} \rightarrow \mathrm{M}$ transformation takes place when $\mu$ reaches a critical value $\mu_{\mathrm{c}}$ (a material constant), the respective transformation stresses for tension and compression are predicted to be,

$$
\begin{aligned}
\sigma^{+} & =\frac{\rho}{\beta^{+}}\left[\Lambda\left(\frac{T}{T_{0}}-1\right)+\mu_{\mathrm{c}}\right], \\
\sigma^{-} & =\frac{\rho}{\beta^{-}}\left[\Lambda\left(\frac{T}{T_{0}}-1\right)+\mu_{\mathrm{c}}\right] .
\end{aligned}
$$

Thus, $\sigma^{+} \beta^{+}=\sigma^{-} \beta^{-}$is a constant for isothermal transformation. Furthermore, eqs. C.5 and C.6 give the Clausius-Clapeyron relations for tension and compression, respectively, as

$$
\begin{aligned}
\frac{\mathrm{d} \sigma^{+}}{\mathrm{d} T} & =\frac{\rho \Lambda}{T_{0} \beta^{+}}, \\
\frac{\mathrm{d} \sigma^{-}}{\mathrm{d} T} & =\frac{\rho \Lambda}{T_{0} \beta^{-}} .
\end{aligned}
$$

These equations predict a larger slope (magnitude) of the transformation stress with temperature for compression than for tension since $\left|\beta^{-}\right|<\beta^{+}$, which is consistent with experiments on similar tubes across various temperatures (Churchill, 2010).

\section{Appendix D. Transformation strains based on Habit Plane Variants}

One can predict the transformation strains from micro-mechanics, so a quantitative comparison with our measurements is done here. The tension-compression asymmetry is generally attributed to the crystallographic asymmetry of the martensitic phase transformation, and many researchers use the set of 24 habit plane variants (HPV) ${ }^{9}$ of martensite to model the superelastic behavior of SMAs (e.g. Patoor et al., 1996; Gall et al., 1999; Gao et al., 2000). We should first mention that this view ignores any detwinning of the habit plane variants that likely occurs past the loading plateaus and is necessarily accompanied by plastic slip. Thus, one should expect the HPV model to apply only to the reversible portion of the superelastic behavior, e.g. excluding the post-plateau portion of the tensile $\mathrm{A} \rightarrow \mathrm{M}^{+}$transformation. As noted in a HPV modeling paper (Jung et al., 2004), the tube drawing process textures the material such that the $\langle 111\rangle_{\mathrm{B} 2}$

\footnotetext{
${ }^{9}$ Also called correspondent variant pairs (CVPs) by others, since each HPV consists of pairs of twin-related lattice correspondence variants (LCVs) of specific volume fractions, about $27 \%$ and $73 \%$ by theory, to achieve an approximately compatible habit plane between austenite and martensite.
} 
direction of the austenite lattice is well aligned with the longitudinal tube axis. Figure $8(\mathrm{~b})$ showed an X-ray map of a tube specimen across the stereographic triangle that did indeed show a prevalent $\langle 111\rangle_{\mathrm{B} 2}$ crystallographic texture aligned to the tube centerline. This texture allows us to approximate the tube as a single crystal to estimate the transformation strains in tension and compression. Using the HPV model, the deformation gradient for $\mathrm{A} \rightarrow \mathrm{M}$ transformation, relative to austenite, is

$$
\boldsymbol{F}^{(k)}=\boldsymbol{I}+g \boldsymbol{m}^{(k)} \otimes \boldsymbol{n}^{(k)}, \quad k=1, \ldots, 24,
$$

where $k$ is the index of a particular HPV, $\boldsymbol{I}$ is the identity tensor, $g$ is the magnitude of the unit cell lattice displacement, $\boldsymbol{n}$ is the habit plane unit normal, $\boldsymbol{m}$ is the unit direction of lattice displacement, and $\otimes$ denotes the outer tensor product of two vectors. We chose the experimental values from Matsumoto et al. (1987) with respect to the B2 frame: $g=0.140, n_{j}^{(1)}=(-0.8684,-0.4138,0.26878)$, and $m_{k}^{(1)}=(0.4580,0.7706,0.4432)$. The other variants are found by permutations of the components, and we adopted the ordering found in Gall et al. $(1999)^{10}$. The driving force for $\mathrm{A} \rightarrow \mathrm{M}$ transformation (per unit reference volume) for each HPV is

$$
\rho_{0} \mu^{(k)}=J \boldsymbol{\sigma}: \varepsilon_{T}^{(k)}=\boldsymbol{N} \cdot \boldsymbol{H}^{(k)},
$$

where $J=\operatorname{det} \boldsymbol{F}$ (close to unity for NiTi), and the following are the respective conjugate transformation strains to the Cauchy stress and Nominal stress tensors: $\varepsilon_{T}=\frac{1}{2}\left(\nabla_{\boldsymbol{x}} \boldsymbol{u}+\left(\nabla_{\boldsymbol{x}} \boldsymbol{u}\right)^{T}\right)$ is the symmetrized (Eulerian) displacement gradient, and $\boldsymbol{H}=\nabla_{\boldsymbol{X}} \boldsymbol{u}=\boldsymbol{F}-\boldsymbol{I}$ is the (referential) displacement gradient.

Assuming the applied stress is purely uniaxial and using the form of the nominal stress of eq. (B.16), the thermodynamic driving force for each variant becomes

$$
\rho_{0} \mu^{(k)}=\boldsymbol{N} \cdot \boldsymbol{H}^{(k)}=\operatorname{tr}\left(\boldsymbol{N} \cdot \boldsymbol{H}^{(k)}\right)=\sigma_{0}^{e}\left(\boldsymbol{e} \cdot \boldsymbol{H}^{(k)} \cdot \boldsymbol{e}_{0}\right) .
$$

Thus, for a given value of $\sigma_{0}^{e}$ the quantity in parentheses plays the role of the conjugate transformation strain $\beta$ as used in eq. (C.4),

$$
\beta^{(k)}=\boldsymbol{e} \cdot \boldsymbol{H}^{(k)} \cdot \boldsymbol{e}_{0}=e_{i} H_{i j}^{(k)} e_{j}^{0},
$$

where the usual summation convention applies for $i$ and $j$ in the Cartesian coordinate system of the austenite frame. The preferred HPV $(l)$ is just selected as the maximum of the set of driving forces

$$
\mu^{(l)}=\max _{k \in[1, \ldots, 24]}\left\{\mu^{(k)}\right\}
$$

and this could be done for each loading direction in the austenite frame to find $l=l\left(\boldsymbol{e}_{0}\right)$, say across the stereographic triangle (but see our comment at the end of this appendix). In the $\langle 111\rangle_{\mathrm{B} 2}$ direction with $\hat{\boldsymbol{e}}_{0}=\{1,1,1\} / \sqrt{3}, \beta^{(l+)}=0.06639$ for tension and $\beta^{(l-)}=-0.03152$ for compression. These are somewhat different from the transformation strains we measured. However, there are in fact six equally preferred HPV's in tension and another six in compression, since $\langle 111\rangle$ is a relatively high symmetry axis for the crystal. This means that several HPV's may be generated with equal probability and be activated simultaneously. Choosing instead the average (homogenized) deformation gradient $\overline{\boldsymbol{F}}$ of the six preferred HPVs, one finds that, unlike the individual HPVs, the ensemble (both for tension and compression) is a rotationless pure deformation, $\overline{\boldsymbol{F}}=\overline{\boldsymbol{U}}$, and $\langle 111\rangle_{\mathrm{B} 2}$ is a principal axis of the deformation. Now, $\boldsymbol{e}=\boldsymbol{e}_{0}$ and the relevant strain measure on average is $\overline{\boldsymbol{U}}-\boldsymbol{I}$ (the Biot strain), so

$$
\beta_{\langle 111\rangle}=\hat{\boldsymbol{e}}_{0} \cdot(\overline{\boldsymbol{U}}-\boldsymbol{I}) \cdot \hat{\boldsymbol{e}}_{0}
$$

This calculation gives $\beta_{\langle 111\rangle}^{+}=0.05470$ for tension and $\beta_{\langle 111\rangle}^{-}=-0.03714$ for compression. These values agree rather well with our uniaxial data $(0.0540$ and -0.0349$)$, especially considering the fact that the tube specimen is not truly a single crystal.

Finally, the impetus to use finite deformation measures was our desire to be as precise as possible in the interpretation of our data. One might argue, however, that infinitesimal strain theory would have

\footnotetext{
${ }^{10}$ Note, however, Gall et al. (1999) used the theoretical values instead of experimental values from Matsumoto et al. (1987).
} 
been sufficiently accurate, considering the moderate transformation strains of NiTi (of order $5 \%$ ). In fact, eq. (D.6) is numerically equivalent to $\hat{\boldsymbol{e}}_{0} \cdot \boldsymbol{H}^{(l)} \cdot \hat{\boldsymbol{e}}_{0}$ calculated from an individual preferred variant, seemingly the same as infinitesimal theory. However, this equivalence stems from our choice to use the average of the six preferred HPVs, which have zero average rotation, and the fact that the stress state is purely uniaxial which extracts only the symmetric part of $\boldsymbol{H}^{(l)}$. We showed above that the effect of finite rotation of a single variant is not that small (a $20 \%$ difference in transformation strain in tension), so the effect warrants careful consideration. Furthermore, the thermodynamic driving force used here to extract the transformation strain was derived from a potential function (the Gibb's free energy), so finite rotations (whether they are included or not) have implications on how one calculates the correct potential energy of the external loading device. We see some pitfalls here. Additionally, it is not obvious how one should calculate the transformation strain, at least in a simple way, for other loading axes. A slight change in loading direction off the $\langle 111\rangle$ axis would lead to a single preferred variant, according to the simplistic prescription of eq. (D.5), which would give a jump in the transformation strain values from those strictly along $\langle 111\rangle$. Such a discontinuity would be rather unexpected, and one would need to consider how compatibility enforces constraints on neighboring material domains to give a more continuous dependence of the macroscopic transformation strain on loading direction. How this homogenization should be properly done, without resorting to extremely detailed calculations, is probably open for debate. 


\section{References}

Adler, P. H., Allen, J., J., L., Francis, R., 2007. Martensite transformations and fatigue behavior of Nitinol. Journal of ASTM International $4(7)$.

Atanacković, T., Achenbach, M., 1989. Moment-curvature relations for a pseudoelastic beam. Continuum Mechanics and Thermodynamics 1 (1), 73-80.

Auricchio, F., Sacco, E., 1997. A superelastic shape-memory-alloy beam model. Journal of intelligent material systems and structures 8 (6), 489-501.

Berg, B., 1995a. Bending of superelastic wires, Part I: Experimental aspects. Journal of Applied Mechanics $62,459$.

Berg, B., 1995b. Bending of superelastic wires, Part II: Application to three-point bending. Journal of Applied Mechanics 62, 466.

Brazier, L., 1927. On the flexure of thin cylindrical shells and other "thin" sections. Proceedings of the Royal Society of London. Series A 116 (773), 104.

Brinson, L. C., Schmidt, I., Lammering, R., 2004. Stress-induced transformation behavior of a polycrystalline NiTi shape memory alloy: micro and macromechanical investigations via in situ optical microscopy. Journal of the Mechanics and Physics of Solids 52 (7), 1549-1571.

Bundara, B., Tokuda, M., Kuselj, B., Ule, B., Tuma, J., 2000. Superelastic tension and bending characteristics of shape memory alloys. Metals and Materials International 6, 293-299, 10.1007/BF03028074.

Buratti, G., 2005. Stresses and deflections in a pseudo-elastic beam under cyclic loads. Continuum Mechanics and Thermodynamics 17 (2), 149-157.

Chang, B.-C., Shaw, J. A., Iadicola, M. A., 2006. Thermodynamics of shape memory alloy wire: Modeling, experiments, and application. Continuum Mechanics and Thermodynamics 18 (1-2), 83-118.

Choi, E., Lee, D., Choei, N., 2009. Shape memory alloy bending bars as seismic restrainers for bridges in seismic areas. Int. J. Steel Struct 9 (4), 261-273.

Churchill, C. B., Dec. 2010. Experimental techniques for characterizing the thermo-electro-mechanical shakedown response of SMA wires and tubes. PhD dissertation, The University of Michigan, Department of Aerospace Engineering.

Correlated Solutions, 2010a. Vic-3D Manual. Correlated Solutions, Inc., Columbia, SC.

Correlated Solutions, 2010b. Vic-3D Testing Guide. Correlated Solutions, Inc., Columbia, SC.

De la Flor, S., Urbina, C., Ferrando, F., 2010. Asymmetrical bending model for NiTi shape memory wires: Numerical simulations and experimental analysis. Strain, 1475-1305.

Dolce, M., Cardone, D., 2005. Fatigue resistance of SMA-martensite bars subjected to flexural bending. International Journal of Mechanical Sciences 47 (11), 1693-1717.

Duerig, T. W. and Pelton, A. and Stöckel, D., 1999. An overview of nitinol Medical applications. Materials Science and Engineering A 273-275, 149-160.

Favier, D., Louche, H., Schlosser, P., Orgeas, L., Vacher, P., Debove, L., Jan 2007. Homogeneous and heterogeneous deformation mechanisms in an austenitic polycrystalline Ti-50.8 at.\% Ni thin tube under tension. investigation via temperature and strain fields measurements. Acta Materialia 55 (16), 5310-5322.

Feng, P., Sun, Q. P., May 2006. Experimental investigation on macroscopic domain formation and evolution in polycrystalline NiTi microtubing under mechanical force. Journal of the Mechanics and Physics of Solids 54, 1568-1603.

Gall, K., Sehitoglu, H., Chumlyakov, Y., Kireeva, I., 1999. Tension-compression asymmetry of the stress-strain response in aged single crystal and polycrystalline NiTi. Acta Materialia 47 (4), 1203-1217.

Gao, X., Huang, M., Brinson, L. C., 2000. A multivariant micromechanical model for SMAs Part 1. Crystallographic issues for single crystal model. International Journal of Plasticity 16 (10-11), 1345-1369.

Hasan, M., Schmahl, W., Hackl, K., Heinen, R., Frenzel, J., Gollerthan, S., Eggeler, G., Wagner, M., Khalil-Allafi, J., Baruj, A., 2008. Hard X-ray studies of stress-induced phase transformations of superelastic NiTi shape memory alloys under uniaxial load. Materials Science and Engineering: A 481, 414-419.

He, Y., Sun, Q., 2010. Macroscopic equilibrium domain structure and geometric compatibility in elastic phase transition of thin plates. International Journal of Mechanical Sciences 52 (2), 198-211.

Humbeeck, J. V., 1999. Non-medical applications of shape memory alloys. Materials Science and Engineering A 273-275, 134148.

Iadicola, M. A., Shaw, J. A., 2002a. The effect of uniaxial cyclic deformation on the evolution of phase transformation fronts in pseudoelastic NiTi wire. Journal of Intelligent Material Systems and Structures 13 (2), 143-156.

Iadicola, M. A., Shaw, J. A., 2002b. An experimental setup for measuring unstable thermo-mechanical behavior of shape memory alloy wire. Journal of Intelligent Material Systems and Structures 13 (2), 157-166.

Irschik, H., Gerstmayr, J., 2009. A continuum mechanics based derivation of Reissner's large-displacement finite-strain beam theory: the case of plane deformations of originally straight bernoulli-euler beams. Acta Mechanica 206 (1), 1-21.

Jung, Y., Papadopoulos, P., Ritchie, R. O., 2004. Constitutive modelling and numerical simulation of multivariant phase transformation in superelastic shape-memory alloys. International Journal for Numerical Methods in Engineering 60, 429460.

Kocks, U., Kallend, J., Wenk, H., Rollett, A., Wright, S., 1995. popLA, preferred orientation package-Los Alamos. Los Alamos National Laboratory, Los Alamos, NM.

Kyriakides, S., Ok, A., Corona, E., 2008. Localization and propagation of curvature under pure bending in steel tubes with Lud̈ers bands. International Journal of Solids and Structures 45, 3074-3087.

Li, Z. Q., Sun, Q. P., 2002. The initiation and growth of macroscopic martensite band in nano-grained NiTi microtube under tension. International Journal of Plasticity 18 (11), 1481-1498. 
Liew, K., Ren, J., Kitipornchai, S., 2004. Analysis of the pseudoelastic behavior of a SMA beam by the element-free galerkin method. Engineering analysis with boundary elements 28 (5), 497-507.

Mao, S., Luo, J., Zhang, Z., Wu, M., Liu, Y., Han, X., 2010. EBSD studies of the stress-induced B2-B19'martensitic transformation in NiTi tubes under uniaxial tension and compression. Acta Materialia 58 (9), 3357-3366.

Matlab R2010b, 2010. Matlab Documentation. MathWorks, Natick, MA.

Matsumoto, O., Miyazaki, S., Otsuka, K., Tamura, H., 1987. Crystallography of martensitic transformation in Ti-Ni single crystals. Acta Metallurgica 35 (8), $2137-2144$.

Mehta, A., Gong, X., Imbeni, V., Pelton, A., Ritchie, R., 2007. Understanding the deformation and fracture of Nitinol endovascular stents using in situ synchrotron X-Ray microdiffraction. Advanced Materials 19 (9), 1183-1186.

Miyazaki, S., Otsuka, K., 1986. Deformation and transition behavior associated with the R-phase in Ti-Ni alloys. Metallurgical and Materials Transactions A 17 (1), 53-63.

Morgan, N., 2004. Medical shape memory alloy applications - the market and its products. Materials Science \& Engineering A $378(1-2), 16-23$.

Ng, K. L., Sun, Q. P., 2006. Stress-induced phase transformation and detwinning in NiTi polycrystalline shape memory alloy tubes. Mechanics of Materials 38 (1), 41-56.

Patoor, E., Eberhardt, A., Berveiller, M., 1996. Micromechanical modelling of superelasticity in shape memory alloys. Journal de Physique(France) IV(France) 6 (1), 277-292.

Pelton, A., Rebelo, N., Duerig, T., Wick, A., 1994. Experimental and FEM analysis of the bending behavior of superelastic tubing. In: SMST-94: Proceedings of the First International Conference on Shape Memory and Superelastic Technologies,(AR Pelton, D. Hodgson and TW Duerig eds.) Pacific Grove, California, USA. p. 353.

Perry, K., Labossiere, P., Steffler, E., 2007. Measurement of deformation and strain in Nitinol. Experimental mechanics 47 (3), 373-380.

Plietsch, R., Bourauel, C., Drescher, D., Nellen, B., 1994. Analytical description of the bending behaviour of NiTi shape-memory alloys. Journal of materials science 29 (22), 5892-5902.

Purohit, P., Bhattacharya, K., 2002. On beams made of a phase-transforming material. International Journal of Solids and Structures 39 (13-14), 3907-3929.

Rajagopal, K., Srinivasa, A., 2005. On the bending of shape memory wires. Mechanics of Advanced Materials and Structures $12(5), 319-330$.

Raniecki, B., Rejzner, J., Lexcellent, C., 2001. Anatomization of hysteresis loops in pure bending of ideal pseudoelastic SMA beams. International journal of mechanical sciences 43 (5), 1339-1368.

Reedlunn, B., Daly, S., Hector, L. G. J., Zavattieri, P., Shaw, J. A., 2013. Tips and tricks for characterizing shape memory alloy wire: Part 5 - full-field strain measurement by digital image correlation. Experimental Techniques 37 (3), $62-78$.

Rejzner, J., Lexcellent, C., Raniecki, B., 2002. Pseudoelastic behaviour of shape memory alloy beams under pure bending: experiments and modelling. International journal of mechanical sciences 44 (4), 665-686.

Schmahl, W., Khalil-Allafi, J., Hasse, B., Wagner, M., Heckmann, A., Somsen, C., 2004. Investigation of the phase evolution in a super-elastic NiTi shape memory alloy (50.7 at.\% Ni) under extensional load with synchrotron radiation. Materials Science and Engineering: A 378 (1), 81-85.

Shaw, J. A., Jan. 1997. Material instabilities in a Nickel-Titanium shape memory alloy. PhD dissertation, The University of Texas at Austin, Department of Aerospace Engineering.

Shaw, J. A., Kyriakides, S., 1995. Thermomechanical aspects of NiTi. Journal of the Mechanics and Physics of Solids 43 (8), $1243-1281$.

Shaw, J. A., Kyriakides, S., 1997. On the nucleation and propagation of phase transformation fronts in a NiTi alloy. Acta Materialia 45 (2), 683-700.

Shaw, J. A., Kyriakides, S., 1998. Initiation and propagation of localized deformation in elasto-plastic strips under uniaxial tension. International Journal of Plasticity 13 (10), 837-871.

Sutton, M. A., Orteu, J. J., Schreier, H. W., 2009. Image Correlation for Shape, Motion, and Deformation Measurements: Basic Concepts, Theory, and Applications. Springer, New York.

Thier, M., Mick, A., Drescher, D., Bourauel, C., 1991. Deformation behaviour of NiTi shape memory alloys in bending. Journal of Materials Science 26 (23), 6473-6478.

Young, M., Wagner, M., Frenzel, J., Schmahl, W., Eggeler, G., 2010. Phase volume fractions and strain measurements in an ultrafine-grained NiTi shape-memory alloy during tensile loading. Acta Materialia 58 (7), 2344-2354. 